Universidade De São Paulo

Instituto DE Física

\title{
Descoberta e Discernimento de Supersimetria versus Dimensões Extras Universais no CERN LHC
}

\author{
Rafael Marcelino do Carmo Silva
}

Orientador: Prof. Dr. Oscar José Pinto Éboli

Co-Orientador: Prof. Dr. Alexandre Alves (UNIFESP)

Banca Examinadora:

Prof. Dr. Oscar José Pinto Éboli (IFUSP)

Prof. Dr. Adilson José da Silva (IFUSP)

Prof. Dr. Enrico Bertuzzo (IFUSP)

Prof. Dr. André Paniago Lessa (UFABC)

Prof. Dr. Eduardo de Moraes Gregores (UFABC)

Tese de Doutorado apresentada ao Instituto de Física para obtenção do título de Doutor em Ciências 


\section{FICHA CATALOGRÁFICA \\ Preparada pelo Serviço de Biblioteca e Informação do Instituto de Física da Universidade de São Paulo}

Silva, Rafael Marcelino do Carmo

Descoberta e discernimento de supersimetria versus

dimensões extras universais do CERN LHC. - São Paulo, 2015.

Tese (Doutorado) - Universidade de São Paulo.

Instituto de Física. Depto. de Física Matemática

Orientador: Prof. Dr. Oscar José Pinto Éboli.

Área de Concentração: Supersimetria

Unitermos: 1. Supersimetria; 2. Teoria de campos; 3.Física de partículas; 4. Fenomenologia; 5. Análise estatística de dados. 


\section{Agradecimentos}

A Oscar Éboli, pelos conselhos e supervisão. A Alexandre Alves pela leitura exaustiva e crítica realizada nos últimos meses, sabemos da nossa dedicação nesse trabalho e que não conseguiria chegar até aqui se não fosse a sua ajuda nesse projeto. Ao CNPq pelo suporte financeiro.

Aos amigos do Instituto de Física pelas ideias, suporte acadêmico e boas conversas. Aos meus amados pais e irmã.

Em especial à minha esposa e filha - pelo suporte, carinho e lições de amor incondicional que passei a receber diariamente com vocês ao meu lado. 
The search for supersymmetry is one of the great dramas in present-day physics.

E. Witten, 2012 


\section{Resumo}

Estimar de forma realista o alcance de descoberta de um experimento de colisão de altas energias, como o realizado no Large Hadron Collider (LHC) do CERN, é uma tarefa complexa, principalmente em vista das técnicas de simulação de eventos e dos métodos de estatística multivariada utilizadas pelas colaborações experimentais na comparação dos dados com as predições teóricas.

Descobrir uma nova partícula, contudo, é apenas o primeiro passo na investigação experimental. De modo a estabelecer qual dos eventuais modelos teóricos concorrentes é favorecido pelos dados, torna-se imprescindível o estudo das propriedades desta nova partícula e de suas interações com o restante do espectro. Informações como os números quânticos de spin, conjugação de carga $(C)$ e paridade $(P)$, podem ser obtidas através do estudo das correlações entre os momentos das partículas produzidas codificadas nas distribuições cinemáticas. O discernimento entre os vários modelos, portanto, passa a ser um problema de combinar todas estas informações de forma eficiente e compará-las aos dados experimentais através de um teste estatístico e decidindo, assim, pela confirmação ou não de um novo sinal e sobre o modelo que melhor explica aqueles dados.

No trabalho realizado nesta tese, investigamos o limite do LHC, operando a uma energia de centro-de-massa de $14 \mathrm{TeV}$, para a descoberta de um modelo supersimétrico (SUSY) simplificado e de seu discernimento em relação a um modelo de dimensões extras universais mínimas (MUED), usando eventos de produção de novas partículas coloridas decaindo, através de cadeias curtas, em jatos e missing energy.

Nossa abordagem avança em diversos aspectos em comparação a fenomenologias mais simplificadas: utilizando uma análise estatística multivariada, levando em conta incertezas sistemáticas nas normalizações das seções de choque e no formato das distribuições, empregando técnicas de identificação de jatos de quarks e glúons para uma melhor separação dos backgrounds do Modelo padrão (MP), escaneando e otimizando os cortes retangulares, simulando eventos de forma cuidadosa e com correções de ordem superior da cromodinâmica quântica (QCD).

Eventos de SUSY e MUED foram simulados para 150 diferentes espectros de massa, ainda não excluídos pelo LHC, e estimamos o potencial de descoberta e de discernimento SUSY versus MUED no plano de massas de squarks e gluinos utilizando as técnicas acima mencionadas. Mostramos, em primeiro lugar, que mesmo de forma simplificada, inserir incertezas sistemáticas é essencial para uma estimativa mais realista do potencial do acelerador, principalmente no que diz respeito ao aumento de luminosidade integrada. Para incertezas nas normalizações da ordem de $20 \%$, o ganho no potencial de busca torna-se mais limitado. Por exemplo, passando de $100 \mathrm{a}$ $3000 \mathrm{fb}^{-1}$, o alcance na massa dos squarks aumenta de 2.8 para $\sim 3.1 \mathrm{TeV}$, ao passo que, sem levar em conta estas incertezas, a estimativa é mais otimista, indo de $3.0 \mathrm{a} \sim 3.5 \mathrm{TeV}$ para as mesmas luminosidades.

Performance similar é observada no discernimento SUSY versus MUED, onde é possível obter uma significância de $5 \sigma$ para massas de squarks de até $\sim 2.7 \mathrm{TeV}$ e gluinos $\sim 5 \mathrm{TeV}$, mantendo-se as incertezas sistemáticas a um nível menor do que $10 \%$ aproximadamente.

De forma geral, concluímos que um modelo supersimétrico simplificado, como o estudado aqui, pode ser descoberto e confirmado (em relação a um dos seus mais populares concorrentes, MUED) para um espectro com squarks, gluinos e neutralinos de aproximadamente 2.5, 5.0 e 0.3 $\mathrm{TeV}$, respectivamente, se as incertezas sistemáticas puderem ser controladas a um nível de $10 \%$ ou menos, após $3 \mathrm{ab}^{-1}$ de luminosidade integrada. 
Palavras-chave: supersimetria, teoria de campos, física de partículas, fenomenologia, análise estatística de dados. 


\section{Abstract}

The problem of estimating, in a realistic way, the reach of an experiment in high energy physics, such as the CERN Large Hadron Collider (LHC), is a difficult task. Specially due to the simulations techniques and the multivariate statistics for data and theory comparisons, used by experimental collaborations.

The discovery of a new particle is just the first step in the experimental exploration. The properties of this particle, like parity, spin and charge are conditions to assert which physics model is favored by the collected data. It is possible to measure these properties with the analyses of the particle momentum correlations through the kinematical distributions. The discriminations among different models turns into a problem of combining all this informations, in a efficient way, and compare with experimental data through a statistical test, and choosing for the confirmation or exclusion of a signal and which model best describes the data.

In this work, we investigate the limits of the LHC, working in a center of mass energy of 14 $\mathrm{TeV}$, for the discovery of a simplified model of supersymmetry (SUSY) and the discrimination with a model of minimal universal extra dimensions (MUED), using productions of heavy colored particles decaying, through short decays chains, in jets and missing energy.

Our approach progresses in different aspects compared with simplified phenomenological analyses: we used a multivariate statistical analysis, considered systematical uncertainties in the rate and shape of distributions, implemented techniques of quarks and gluons jet tagging identification for a good separation between signal and backgrounds, scanning for the best rectangular cuts and simulating events in a careful way with 1-loop corrections from quantum chromodynamics.

Our events were simulated for 150 different mass spectrums, not excluded by the LHC, and we estimate the potencial for discovery and discrimination of SUSY versus MUED in a squarksgluinos mass plane, using the techniques mentioned above. We proved, in first place, that even in a simplified way, inserting systematical uncertainties it's essential for an estimative more realistic of the collider's reach, mainly with the increasing of integrated luminosity. For systematical rate uncertainties in the distribution of $20 \%$, the gain in the discovery potencial is very limited. For example, increasing from 100 to $3000 \mathrm{fb}^{-1}$, the reach in the squark mass increase from $\sim 2.8$ to $3.1 \mathrm{TeV}$. On the other hand, without systematical uncertainties in rate distributions, the reach is more optimistic, from $3.0 \mathrm{TeV}$ to $\sim 3.5 \mathrm{TeV}$, for the same luminosities.

Similar performance was observed in the discrimination of Susy versus MUED, where it's possible to obtain significance of $5 \sigma$ for squark masses up to $\sim 2.7 \mathrm{TeV}$ and gluinos of $\sim 5 \mathrm{TeV}$, keeping systematical uncertainties at a level about $10 \%$.

In general, we conclude that a supersymmetryc model, like we studied here, can be discovered and confirmed (compared to one of its more popular competitors, MUED) for a mass spectrum of squarks, gluinos and neutalinos about 2.5, 5.0 and $0.3 \mathrm{TeV}$, respectively, if it's possible to control the systematical uncertainties at a level about $10 \%$, after $3 \mathrm{ab}^{-1}$ of integrated luminosity. 
Key-words: supersymmetry, field theory, particle physics, phenomenology, statistical data analysis. 


\section{Sumário}

\begin{tabular}{lll}
\hline & Introdução & 3
\end{tabular}

\begin{tabular}{lll}
\hline 2 & Revisão Teórica & $\mathbf{7}$
\end{tabular}

$2.1 \quad$ Limitações do Modelo Padrão de partículas $\ldots \ldots \ldots \ldots$. . . . . . . . . . . 7

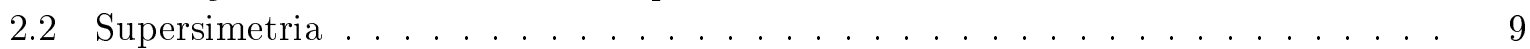

$2.2 .1 \quad$ Paridade-R . . . . . . . . . . . . . . . . . . . . . . . . . . . 11

2.2 .2 Quebra de supersimetria . . . . . . . . . . . . . . . . . . 12

$2.2 .3 \quad$ Lagrangiana supersimétrica . . . . . . . . . . . . . . . . . . . . . . . . 13

2.2 .4 Limites para supersimetria . . . . . . . . . . . . . . . . . . . 14

2.3 Massas de squarks e gluinos $\ldots \ldots \ldots \ldots \ldots \ldots \ldots$

2.4 Dimensões extras universais mínimas . . . . . . . . . . . . . . . . . . . 16

$2.4 .1 \quad$ Paridade-KK $\ldots \ldots \ldots \ldots \ldots \ldots$

$2.4 .2 \quad$ Limites para MUED e massas de KK-quarks e KK-glúons . . . . . . . . . 18

$2.4 .3 \quad$ Lagrangiana de MUED $\ldots \ldots \ldots \ldots \ldots \ldots$

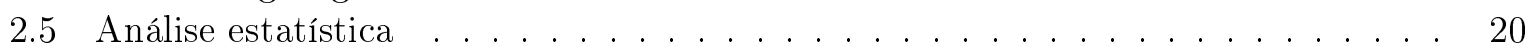

2.6 Construção dos histogramas de verossimilhança . . . . . . . . . . . . . . . . 21

$2.7 \quad$ Estatística-teste para descoberta e discernimento . . . . . . . . . . . . . . 22

$2.8 \quad$ Métricas de significância, $Z_{s b}$ e $Z_{L L R} \ldots \ldots \ldots \ldots \ldots \ldots$

2.8.1 A significância calculada com base na estatística de verossimilhança . . . 25

2.9 Marginalização dos erros sistemáticos $\ldots \ldots \ldots \ldots \ldots \ldots \ldots \ldots$

$\begin{array}{lll}3 & \text { Análise } & 30\end{array}$

3.1 Produção de squarks no LHC $\ldots \ldots \ldots \ldots \ldots \ldots$. . . . . . . . . . . . . 31

3.2 Produção de KK-quarks e KK-glúons no LHC . . . . . . . . . . . . . . . . . . . . 34

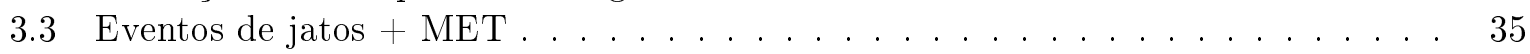

3.4 Produção dos espectros de nova física . . . . . . . . . . . . . . . . . . 35

3.5 Canais para simulação de eventos . . . . . . . . . . . . . . . . . 37

3.5.1 Supersimetria . . . . . . . . . . . . . . . . . 37

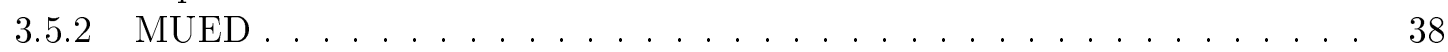

3.5.3 Backgrounds. . . . . . . . . . . . . . . . . . . . . . . . 39

3.6 Simulação de eventos . . . . . . . . . . . . . . . . . . . . . . . 40

3.6.1 Matching de jatos . . . . . . . . . . . . . . . . . . 41

3.6.2 $\quad$ Subtração de gluinos ressonantes . . . . . . . . . . . . . . . . . . 43

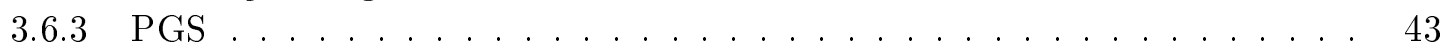

3.7 Seleção de eventos . . . . . . . . . . . . . . . . . . . . . 43

3.8 Observáveis físicos para jatos e MET . . . . . . . . . . . . . . . . . . . . . . . . 45

3.9 Escaneamento de cortes retangulares $\ldots \ldots \ldots \ldots \ldots$

3.10 Correções NLO, Prospino $\ldots \ldots \ldots \ldots \ldots$. . . . . . . . . . . . . . 49

3.11 Identificação de jatos de quarks e glúons (Tagging) . . . . . . . . . . . . . . 51

3.12 Distribuições finais, aplicação de tagging, K-factors e normalização . . . . . . . . . 52

3.13 Incertezas sistemáticas no log-likelihood ratio $\ldots \ldots \ldots \ldots$. . . . . . . . 54 


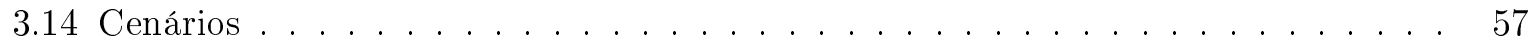

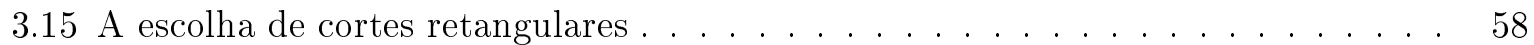

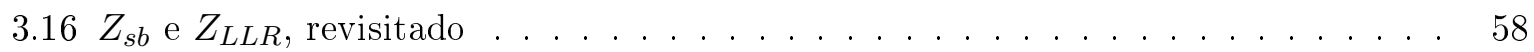

3.17 A escolha do tagging de jatos de quarks e glúons $\ldots \ldots \ldots \ldots$. . . . . . . 61

\begin{tabular}{|ll|l}
\hline 4 & Resultados & 65
\end{tabular}

4.1 Discussão dos resultados no espaço de massas $\ldots \ldots \ldots \ldots$. . . . . . . . . 66

$4.1 .1 \quad$ Descoberta de supersimetria . . . . . . . . . . . . . . . . . . 66

$4.1 .2 \quad$ Discernimento de supersimetria e MUED . . . . . . . . . . . . . . . . 68

$4.1 .3 \quad$ Descoberta e posterior discernimento de nova física . . . . . . . . . . . . . 70

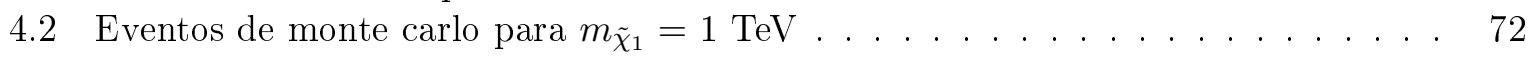

$4.3 \quad$ Formato das distribuições para diferentes $m_{\tilde{\chi}_{1}} \ldots \ldots \ldots \ldots$. . . . . . . 73

\begin{tabular}{lll}
\hline Conclusão & 74
\end{tabular}

\begin{tabular}{|ll}
\hline A Obtenção das Lagrangianas & $\mathbf{7 6}$ \\
\hline
\end{tabular}

A.1 Notação . . . . . . . . . . . . . . . . . . . . . . 76

A.2 Supersimetria . . . . . . . . . . . . . . . . . . . . . . . . . . . 78

A.3 MUED . . . . . . . . . . . . . . . . . . . . . . . . . . . . . . . . . . . 81

A.4 Detalhes de Simulação . . . . . . . . . . . . . . . . . . . . . . . 85 


\section{Capítulo 1}

\section{Introdução}

Dois anos após sua inauguração, os experimentos de colisão próton-próton de altas energias do LHC no CERN alcançaram um dos seus principais objetivos - a descoberta de uma partícula escalar de CP-par, um bóson de Higgs, o último ingrediente necessário para a consistência do Modelo Padrão de Partículas. Há, contudo, fortes argumentos teóricos em favor de uma teoria além do Modelo Padrão, uma vez que este ainda deixa em aberto diversas questões como a própria escala da massa do bóson de Higgs recém descoberto e da existência de matéria escura, por exemplo, entre outras questões. Isso gera uma expectativa de que o LHC possa descobrir alguma forma de nova física em breve.

Existem vários modelos de nova física que abordam muitas destas questões, especialmente a questão da naturalidade do modelo em relação à massa do bóson de Higgs e de candidatos viáveis à matéria escura. Entre estes, sem dúvida, a supersimetria tem o maior apelo teórico por sua abrangência e elegância [1, 2, 3]. Outras sugestões, como a das dimensões extras, propõem solucionar os mesmos problemas de formas diferentes [4, 5, 6, 7]. Contudo, ainda que as soluções sejam essencialmente diferentes, estes dois modelos, especificamente, preveem a existência de espectros de partículas (na escala de até alguns $\mathrm{TeV}$ ) quase que univocamente correspondentes, ainda que seus espectros de massa típicos em cada teoria difiram consideravelmente. Tais partículas podem ter propriedades muito diferentes em relação aos seus números quânticos e acoplamentos também.

Modelos supersimétricos e de dimensões extras universais, equipadas com simetrias apropriadas, preveem a presença de LSPs e LKPs (lightest supersymmetric particles e light Kaluza-Klein particles) no estado final do processo de produção de qualquer partícula nova. Como os detectores não têm capacidade de acusar a presença de uma LSP (LKP), os canais de procura por novas partículas de SUSY e MUED contém alguma combinação de missing energy, jatos e léptons duros. Na classe de modelos que iremos estudar, a dificuldade reside no fato de que as partículas pesadas decaem da mesma forma, levando a assinaturas experimentais, em princípio, parecidas.

A presença de missing energy nos eventos impede a reconstrução do 4-momento das partículas que originaram a cadeia de decaimento, ainda que seja possível inferir a massa delas através de distribuições cinemáticas que exibem limiares [8, 9]. Isso, contudo, demanda uma boa reconstrução destas distribuições e isso só é possível com muitos eventos. Da mesma forma, a informação que se obtém pelo tamanho das seções de choque não constitui mais do que uma evidência em favor de um modelo ou outro. Sem a possibilidade de identificar o modelo de nova física a partir do tamanho das seções de choque e do espectro, seja com eventos de jatos + MET (missing energy), ou outra topologia, só resta o estudo das distribuições cinemáticas disponíveis.

Em cadeias longas, além das distribuições angulares, diferenças ou assimetrias entre distribuições de massas invariantes de conjuntos de partículas do estado final, como jatos e léptons carregados, podem ajudar a discernir entre modelos, veja [10, 11], por exemplo. Com cadeias curtas, como no decaimento direto de sbottoms [12] e de sléptons [13, 14], é possível estudar variáveis angulares correlacionadas com o ângulo de espalhamento do squark, ou slepton. Nesse 
trabalho, escolhemos trabalhar com as distribuições angulares de jatos produzidos no decaimento de squarks e gluinos, aproveitando o número muito maior de eventos esperados em relação a sléptons ou na produção de sbottoms apenas.

Até o momento, no entanto, as análises de 7 e $8 \mathrm{TeV}$ no LHC só puderam excluir regiões dos espaços de parâmetros de teorias além do Modelo Padrão. Em relação à SUSY, squarks e gluinos de até 1.8 e $1.8 \mathrm{TeV}$, aproximadamente, estão excluídos em modelos de quebra soft de supersimetria relativamente simples, como o mSugra, ou assumindo modelos simplificados que não consideram todo o espectro de SUSY mas apenas o setor fortemente interagente, por exemplo, esses limites caem para 1.0 TeV e $1.3 \mathrm{TeV}$ para squarks e gluinos, respectivamente. Isso tem, inclusive, suscitado diversas discussões acerca da naturalidade dos modelos supersimétricos. Vale lembrar contudo, que modelos naturais, ou seja, não finamente ajustados para a massa do Higgs, podem perfeitamente existir em modelos de quebra soft de supersimetria mais complicados.

O nosso trabalho enfoca modelos simplificados de supersimetria e dimensões extras universais. Esses modelos são gerados em um contexto de teoria efetiva de campos, incluindo todos os termos da lagrangiana relevantes para a produção de jatos e missing energy. Nesses modelos é possível estudar a manifestação de supersimetria e dimensões extras universais em um contexto menos restrito. Isso é desejável, pois tratam-se de modelos em bases mais independentes, uma vez que os modelos mais simples de quebra de supersimetria ou com esquemas conhecidos de compactificação de dimensões extras mostram-se cada vez menos prováveis. Dessa forma, não assumimos nenhum modelo de quebra soft específico, nos restringindo a assumir espectros de massa fenomenologicamente interessantes e acessíveis ao LHC $14 \mathrm{TeV}$.

Nossa postura diante do objetivo de discernir SUSY e MUED é maximamente conservadora. Vamos assumir o cenário mais difícil possível de discernimento, onde supomos que as seções de choque de produção e razões de decaimento das partículas de SUSY e MUED são idênticas e nos baseando somente no formato de distribuições cinemáticas para o discernimento. As diferenças encontradas entre as distribuições dos dois modelos são devidas quase exclusivamente ao seus spins, portanto, nosso trabalho pode ser encarado como uma determinação do spin das partículas supersimétricas. A única concessão em favor de supersimetria é que estudamos, na maioria das vezes, espectros típicos desse modelo, apesar de que também investigamos porções do espaço de parâmetros onde as massas são mais degeneradas, caso típico dos espectros de MUED.

Do ponto de vista técnico, estimar o alcance de um experimento de altas energias na procura e discernimento de modelos é uma tarefa que pode ser realizada com vários graus de sofisticação. Análises fenomenológicas mais simples podem ser realizadas, por exemplo, ao nível de partons apenas, dispensando a simulação da hadronização e identificação de jatos e também sem incluir efeitos que diminuem o poder de deteção das partículas. Hoje em dia, contudo, há ferramentas de simulação que permitem gerar eventos que se parecem mais com os eventos reais observados nos detectores. Outro aspecto que vem sendo paulatinamente melhorado nos estudos fenomenológicos encontrados na literatura é o da análise estatística. Ainda que possa dar uma ideia do potencial de descoberta de uma partícula, a métrica de significância normalmente usada em muitos estudos: $S / \sqrt{B}$, onde $S(B)$ denota o número de eventos de sinal (background) observados, pode levar a uma enorme superestimação da significância estatística, por exemplo, no regime onde a distribuição de Poisson, associada a experimentos de contagem de eventos, deve ser usada ao invés de sua aproximação Gaussiana.

Outro aspecto normalmente negligenciado é o de estimar o impacto das incertezas sistemáticas no alcance do experimento. Muitas vezes, isso pode ser feito através de uma simples modificação da métrica de significância: $S / \sqrt{B+\left(\varepsilon_{\text {sys }} B\right)^{2}}$, assumindo uma incerteza sistemática $\varepsilon_{\text {sys }}$ no número de eventos de background. Novamente, ainda que não seja adequada em todas as ocasiões, já impede uma superestimação muito grande do potencial de descoberta.

Delegar sempre a tarefa de estimar (ainda que simplificadamente) o impacto destas incertezas às colaborações experimentais é abdicar de realizar uma análise mais útil tanto para o teórico quanto para o experimental. Levar em conta estas incertezas pode, de imediato, melhorar o 
estudo fenomenológico propondo estratégias mais otimizadas. Por outro lado também trazem novos desafios. Para superar as limitações eventualmente impostas pelos sistemáticos, estatísticas mais poderosas para os testes de hipóteses podem ser usadas, por exemplo, numa análise multivariada.

Neste trabalho, usamos uma estatística baseada no log likelihood ratio, o logaritmo da razão de verossimilhanças: $\Lambda$. Esta estatística combina a informação do formato dos histogramas de diversas distribuições cinemáticas em uma única quantidade, cuja distribuição estatística é obtida de forma simulada, realizando uma grande quantidade de pseudo-experimentos. O lema de Neyman-Pearson [17] garante que um teste de hipóteses simples (onde não há parâmetros a serem ajustados e na ausência de incertezas sistemáticas) baseado em $\Lambda$ tem máximo poder possível (power). A partir, então, das distribuições de probabilidade de $\Lambda$ para eventos de backgrounds e de sinal+backgrounds, são realizados os testes de hipóteses habituais para calcular, por exemplo, a quantidade de dados necessária para uma descoberta. As incertezas sistemáticas, por sua vez, são incorporadas nesta estatística em um esquema híbrido frequentista-Bayesiano, numa espécie de marginalização dos parâmetros relacionados a estas incertezas. Na prática, ainda que o lema de Neyman-Pearson não se aplique devido aos sistemáticos, observa-se que a estatística de log likelihood ratio é uma das melhores para o discernimento de modelos.

Inserimos incertezas sistemáticas no número estimado de eventos de sinal e de background, provenientes, por exemplo, de incertezas na luminosidade integrada e da escolha das escalas de fatorização e renormalização. Em especial, a produção de squarks e gluinos muito pesados envolvem frações grandes de energia e momento dos partons, e que são ainda pobremente estimadas e codificadas nas distribuições de partons disponíveis atualmente. Tais incertezas também foram levadas em conta em nossa simulação. Além disso, incertezas na forma das distribuições, devido a uma baixa eficiência de Monte-Carlo para os backgrounds, após cortes retangulares duros, foram incorporadas também.

A separação entre eventos de sinal e de backgrounds é, em todos os aspectos, uma questão central em uma análise fenomenológica. A forma mais simples, direta e transparente de fazer isso é impor cortes a quantidades observáveis que podem ser construídas com os 4-momentos das partículas, por exemplo, seus momentos transversos, rapidez e massas invariantes. Decidir tais cortes pode ser feito apenas com uma análise visual das distribuições daquelas observáveis. Isso, contudo, não é a abordagem ótima do problema. Escanear o espaço de observáveis de corte à procura de uma região mais rica de sinais é a estratégia mais simples depois da abordagem visual, ainda que muito mais dispendiosa do ponto de vista computacional. Esta foi a abordagem adotada nesta tese e será melhor explicada oportunamente.

Técnicas de subestrutura de jatos têm sido utilizadas recentemente para auxiliar a classificação de eventos de decaimento hadrônico de bósons de gauge, do bóson de Higgs e de quarks top [18, 19, 20]. Isso permite colecionar um número muito maior de eventos ao mesmo tempo que fornece uma maneira de distinguir jatos provenientes dos decaimentos destas partículas e jatos provenientes de radiação de QCD, os quais constituem um enorme background para diversos processos de nova física. Em especial, técnicas de tagging para distinguir entres jatos de quarks e jatos de glúons foram desenvolvidas recentemente [21] e estudadas, inclusive, pelo ATLAS [22].

De forma a auxiliar a remoção de eventos de backgrounds de QCD, utilizamos um tagging de jatos de quarks e glúons de forma parametrizada, a exemplo do que fazemos quando estamos interessados em identificar jatos de quarks bottom. Mostraremos que o uso dessa técnica permite uma separação adicional de eventos de sinal e backgrounds benéfica à descoberta e discernimento entre SUSY e MUED.

Realizar esta análise em um universo de 150 espectros de massa distintos, num total de 900 simulações de eventos de SUSY e MUED, demandou um enorme esforço computacional. Sem uma quase completa automação e sequenciamento de todas as fases de simulação e análise, tal tarefa teria sido praticamente impossível ou, no mínimo, passível de muitos erros. Ao longo da tese apresentaremos aspectos específicos do ponto de vista computacional que foram importantes 
para obter nossos resultados.

A tese está dividida da seguinte forma, no capítulo 2 faremos uma abordagem dos modelos de SUSY e MUED, explicitando especificamente o setor forte da QCD responsável pela produção de jatos + MET. Falaremos sobre os principais avanços experimentais na busca da cada um desses modelos e discutiremos a abrangência dos modelos simplificados que iremos utilizar. Finalizaremos o capítulo com uma discussão sobre a análise estatística empregada neste trabalho, com comparações entre a usual análise de contagem de eventos e a análise multivariada empregada nesta tese.

No capítulo 3 iremos expor o tipo de eventos que analisaremos, os observáveis físicos utilizados em nossa análise multivariada, as simulações para geração de eventos, assim como as técnicas utilizadas para obtenção dos resultados, como: escaneamento de cortes retangulares, tagging de jatos de quarks e glúons e a aplicação de incertezas sistemáticas. Sempre que possível, iremos contrapor a métrica de significância para a simples e amplamente utilizada contagem de eventos $\left(Z_{s b}\right)$ com a métrica da análise multivariada $\left(Z_{L L R}\right)$.

No capítulo 4 iremos expor os resultados obtidos e diversas discussões sobre o comportamento observados das regiões de descoberta. Finalizaremos com a conclusão dada no capítulo 5. 


\section{Capítulo 2}

\section{Revisão Teórica}

Nesse capítulo iremos abordar os pontos fracos do Modelo Padrão de Partículas. Posteriormente iniciaremos a descrição dos modelos de supersimetria e dimensões extras universais mínimas (MUED), com enfoque maior nos termos da lagrangiana responsáveis pela produção de jatos e MET. Finalizaremos o capítulo com a apresentação da estatística de contagem de eventos e log-likelihood ratio, apresentando a forma de obtenção da métrica de significância em cada caso. Logo em seguida introduziremos o conceito de marginalização de likelihoods para análise de incertezas sistemáticas nas taxas e formato das distribuições.

\subsection{Limitações do Modelo Padrão de partículas}

O Modelo Padrão de Partículas, denotaremos como MP daqui em diante, oferece até hoje descrições muito precisas de diversos fenômenos em física de partículas. Porém, conhecemos alguns casos onde a descrição dada pelo Modelo Padrão é incompleta. Nessa seção iremos descrever alguns desses problemas.

\section{Candidato Natural à Matéria Escura}

Baseado em observações astrofísicas [23], notou-se uma discrepância entre a velocidade de rotação de certas galáxias e a massa bariônica nela contida, essa discrepância pode ser resolvida assumindo que existe uma quantidade de massa excedente não interagente e não relativística. Essa quantidade de matéria, que não interage com fótons, foi medida pela colaboração WMAP [28] e supera em cerca de quatro vezes a quantidade de matéria bariônica. Essa matéria é denominada matéria escura. Se, além de interagir gravitacionalmente, a matéria escura interagir fracamente, através de alguma força fraca e de curto alcance, ou por intermédio do bóson de Higgs, por exemplo, é possível explicar a sua abundância nos dias de hoje [25]. O Modelo Padrão não oferece uma partícula que possa representar esse tipo de matéria, ainda que os neutrinos componham uma pequena parte de sua composição. Teorias como supersimetria e dimensões extras, em suas versões mínimas e munidas de uma específica simetria discreta, conseguem fornecer candidatos à matéria escura.

\section{Massa Não-Nula para Neutrinos}

A detecção de oscilação de neutrinos leva a possíveis estados massivos para essas partículas [26, 27]. No Modelo Padrão temos neutrinos de quiralidade Left, porém um neutrino com quiralidade Right teria todos os seus números quânticos de gauge como singletos do grupo $S U(3) \times S U(2)_{L} \times U(1)_{Y}$. Por isso a completa definição de um termo de massa para os neutrinos torna-se complicada no Modelo Padrão. Algumas teorias supersimétricas assumem neutrinos com massa não-nula, isso pode ser feito introduzindo alguns tipos novos de interações [29]. Modelos de dimensões extras também podem acomodar neutrinos de massa não nula [4]. 


\section{Constante Cosmológica}

A constante cosmológica pode ser entendida como uma energia de vácuo que expande nosso universo aceleradamente. O valor estimado da constante cosmológica hoje é bem pequeno $\left(\sim 10^{-30} \mathrm{~g} / \mathrm{cm}^{3}\right)$ e positivo [30], o Modelo Padrão não tem como explicar esse valor para a constante cosmológica. Modelos como supersimetria ou dimensões extras também não conseguem explicar um valor tão pequeno e positivo para a energia de vácuo. A constante cosmológica hoje é um dos maiores desafios para a Física Fundamental.

\section{Assimetria Matéria/Anti-matéria}

O Universo que observamos hoje é constituído basicamente de matéria bariônica. Explicar como essa quantidade de matéria sobreviveu à aniquilação de bárions e anti-bárions em um universo primordial pode ser feita assumindo violação CP no setor de quarks do Modelo

Padrão. Essa violação é observada experimentalmente, porém seu valor é muito pequeno e não consegue reproduzir a quantidade de matéria bariônica observada nos dias de hoje [31]. Alguns modelos supersimétricos conseguem explicar essa assimetria [32].

\section{Problema de Ajuste Fino ou Fine Tuning}

Quando partimos para o domínio da Teoria Quântica de Campos, teorias como o Modelo Padrão possuem divergências ultra-violetas que são removidas por renormalização. Massas, acoplamentos e funções de onda precisam ser redefinidos de modo que seus valores tenham um comportamento não divergente quando analisados no regime de altas energias [33].

O bóson de Higgs é uma partícula escalar de massa $\sim 125 \mathrm{GeV}$ [34]. Assim como qualquer outra partícula do Modelo Padrão, o bóson de Higgs recebe contribuições para sua massa vindas de correções quânticas (1-loop). Se assumirmos a validade do MP até uma escala, digamos $\Lambda_{U V}=10 \mathrm{TeV}$, então deve-se retirar as contribuições vindas do MP acima dessa escala, isso é feito "cortando"os loops do MP para essa energia. As contribuições mais relevantes para a massa do bóson de Higgs são os loops vindos de quark top, bósons de gauge $S U(2) \times U(1)$ e do próprio Higgs, veja Fig. 2.1. As contribuições quadráticas em $\Lambda_{U V}=10 \mathrm{TeV}$ desses loops são:

- Para o loop de quark top,

$$
-\frac{3}{8 \pi^{2}} \lambda_{t}^{2} \Lambda_{U V}^{2} \sim-(2 \mathrm{TeV})^{2}
$$

onde $\lambda_{t}$ é o acoplamento de Yukawa para o quark top.

- Para o loop de bósons gauge,

$$
\frac{g^{2}}{16 \pi^{2}} \Lambda_{U V}^{2} \sim(0.7 \mathrm{TeV})^{2}
$$

onde $g^{2} \sin ^{2} \theta_{W}=e^{2}$, onde $e$ é a carga elétrica e $\sin \theta_{W}=0.23$ é o ângulo de rotação induzido após quebra de simetria eletro-fraca para os campos de gauge $W^{0}$ e $B^{0}$.

- Para o loop do bóson de Higgs,

$$
\frac{\lambda^{2}}{16 \pi^{2}} \Lambda_{U V}^{2} \sim(0.5 \mathrm{TeV})^{2}
$$

$\lambda$ é o acoplamento quártico de Higgs.

Assim a massa total para o bóson de Higgs será dada, aproximadamente, por:

$$
m_{h}^{2}=m_{\text {ărvore }}^{2}-(100-10-5)(200 \mathrm{GeV})^{2} .
$$

Com a massa do Higgs da ordem de $100 \mathrm{GeV}$, um ajuste fino de 1 em 100, entre os acoplamentos a nível de árvore, é necessário. Se aumentarmos para $\Lambda_{U V}=100 \mathrm{TeV}$, o ajuste passa para 1 parte em 10000 . Porém se a escala estiver por volta de $1 \mathrm{TeV}$, nenhum ajuste fino é necessário. 


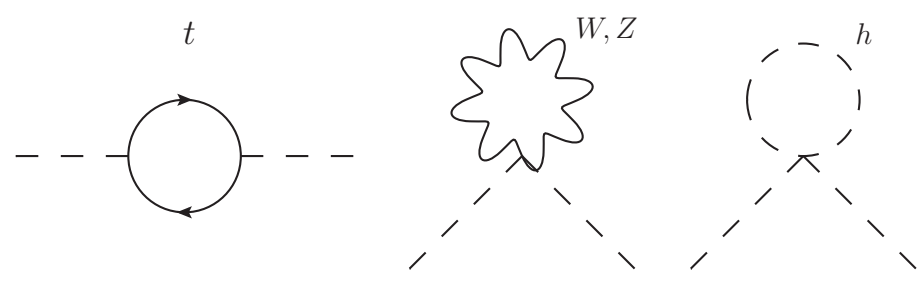

Figura 2.1: Diagramas em 1-loop que contribuem majoritariamente para divergências quadráticas para a massa do bóson de Higgs no Modelo Padrão.

\subsection{Supersimetria}

Supersimetria é uma simetria do espaço-tempo que relaciona bósons e férmions através de um operador fermiônico $Q$,

$$
\begin{aligned}
& Q \mid \text { bóson }>=\mid \text { férmion }>, \\
& Q \mid \text { férmion }>=\mid \text { bóson }>.
\end{aligned}
$$

Em versões mínimas, cada partícula será associada com um parceiro supersimétrico de spin $\pm \frac{1}{2}$ em um supermultipleto. Todas as partículas do supermultipleto terão a mesma massa e número quânticos de gauge, pois o operador $Q$ comuta com todos os geradores do grupo de gauge $\left(S U(3)_{C} \times S U(2)_{L} \times U(1)_{Y}\right)$. Além disso, os supermultipletos possuem o mesmo número de graus de liberdade bosônicos e fermiônicos [1, 3, 2,

Os supermultipletos são definidos em duas grandes classes, os quirais e os vetoriais. Para definirmos o Modelo Padrão supersimétrico teremos que introduzir um conjunto de supermultipletos quirais para definir os quarks e léptons. E um conjunto de supermultipletos vetoriais para os bósons de gauge.

Cada quark e lépton é associado a um supermultipleto quiral juntamente com seu parceiro supersimétrico de spin-0, os squarks e sléptons $(\tilde{q}, \tilde{l})$. Sabemos que as componentes Left e Right dos quarks e léptons possuem números quânticos diferentes no Modelo Padrão. Por isso teremos que associar essas componentes separadamente em supermultipletos diferentes. Por exemplo, teremos um supermultipleto para o quark up Right e outro supermultipleto para os quarks up Left. O que denota que para um quark up teremos dois parceiros supersimétricos de spin-0. O mesmo para os outros quarks e léptons do Modelo Padrão.

Os supermultipletos vetoriais recebem os bósons de gauge $\left(W^{ \pm}, W^{3}, B, g\right)$, novamente para cada um desses bósons teremos um parceiro supersimétrico de spin- $1 / 2$, os gauginos (winos $\left(\tilde{W}^{ \pm}, \tilde{W}^{0}\right)$, binos $\tilde{B}^{0}$ e gluinos $\left.\tilde{g}\right)$.

O bóson de Higgs também será representado por um supermultipleto quiral. Porém supersimetria traz uma dificuldade quanto ao mecanismo de Higgs. No formalismo de supercampos não é possível acoplar um supermultipleto para o bóson de Higgs com os quarks up, charm e top ao mesmo tempo que acopla-se com os quarks down, bottom e strange. Isso ocorre porque em potenciais supersimétricos que dão origem aos acoplamentos de Yukawa não podem conter termos complexos conjugados para o supermultipleto de Higgs, ditos termos não-holomórficos [1, 2]. Assim, pelo menos dois supermultipletos de Higgs devem ser inseridos para a realização do mecanismo de Higgs, $H_{u}$ e $H_{d}$. Com isso teremos dois escalares de Higgs neutros e dois carregados $\left(H_{u}^{0}, H_{d}^{0}, H_{u}^{+}, H_{d}^{-}\right)$, consequência da inserção de dois supermultipletos quirais, e os seus parceiros supersimétricos, chamamos de Higssinos. Após a quebra espontânea de simetria eletro-fraca esses bósons dão origem ao bóson de Higgs (escalar leve com CP-par), dois escalares neutros, e dois carregados.

As partículas listadas até agora não são necessariamente os estados de massa do Modelo Padrão supersimetrizado. Há misturas em setores, como entre os gauginos e higgsinos no setor eletro-fraco, e entre squarks/sléptons. O bino, zino e dois higssinos neutros misturam-se e formam 
4 estados denominados neutralinos $\tilde{\chi}_{1,2,3,4}^{0}$. Já a mistura de winos e higgsinos carregados geram dois charginos $\tilde{\chi}_{1,2}^{ \pm}$. No setor de squarks e sléptons a mistura é proporcional à massa da partícula parceira original do Modelo Padrão. Nesses casos apenas misturas para squarks top, bottom e staus são relevantes. Os stops (sbottoms) Right e Left misturam-se e resultam em dois estados $\tilde{t}_{1}$ e $\tilde{t}_{2}\left(\tilde{b}_{1}\right.$ e $\left.\tilde{b}_{2}\right)$. O mesmo para o stau.

Munidos de alguns ingredientes a mais que discutiremos nas próximas seções, temos o que chamamos de MSSM (minimal supersymmetric standard model ou modelo padrão supersimétrico mínimo). Na Tab. 2.1 e 2.2 temos todas as partículas para o MSSM com seus respectivos números quânticos de gauge.

\begin{tabular}{cccc}
\hline \multicolumn{5}{c}{ Supermultipletos Quirais } \\
\hline & spin-0 & spin-1/2 & $S U(3)_{c}, S U(2)_{L}, U(1)_{Y}$ \\
\hline squarks, quarks & $\tilde{q}_{L}^{u}$ e $\tilde{q}_{L}^{d}$ & $\left(q^{u} q^{d}\right)_{L}$ & $\left(3,2, \frac{1}{3}\right)$ \\
& $\tilde{q}_{R}^{u}, \tilde{q}_{R}^{d}$ & $q_{R}^{u}, q_{R}^{d}$ & $\left(3,0, \frac{4}{3} /-\frac{2}{3}\right)$ \\
sléptons, léptons & $\tilde{\nu}$ e $\tilde{l}_{L}$ & $(\nu l)_{L}$ & $(1,2,-1)$ \\
& $\tilde{l}_{R}$ & $l_{R}$ & $(1,1,-2)$ \\
\hline \multicolumn{5}{c}{ Supermultipletos Vetoriais } \\
gluinos, glúons & spin-1/2 & spin-1 & $S U(3)_{c}, S U(2)_{L}, U(1)_{Y}$ \\
winos e bósons W & $\tilde{W}^{ \pm}, \tilde{W}^{0}$ & $g$ & $(8,1,0)$ \\
binos e bóson B & $\tilde{B}^{0}$ & $W^{ \pm}$ & $(1,3,0)$ \\
\hline
\end{tabular}

Tabela 2.1: Partículas oriundas dos supermultipletos quirais e vetoriais para o MSSM. A hipercarga é dada por $Q=T_{3}+\frac{Y}{2} \cdot q^{u}, q^{d}$ indicam quarks do tipo up e down, respectivamente.

\begin{tabular}{ccc}
\hline \multicolumn{3}{c}{ Estados Diagonalizados de Massas } \\
\hline & não diagonalizados & diagonalizados \\
neutralinos & $\tilde{B}^{0}, \tilde{W}^{0}, \tilde{H}_{u}{ }^{0}, \tilde{H}_{d}{ }^{0}$ & $\tilde{\chi}_{1,2,3,4}$ \\
charginos & $\tilde{W}^{ \pm}, \tilde{H}_{u}^{+}, \tilde{H}_{d}^{-}$ & $\tilde{\chi}_{1}^{ \pm}, \tilde{\chi}_{2}^{ \pm}$ \\
stops, sbottoms & $\left(\tilde{t}_{R}, \tilde{t}_{L}\right),\left(\tilde{b}_{R}, \tilde{b}_{L}\right)$ & $\left(\tilde{t}_{1}, \tilde{t}_{2}\right),\left(\tilde{b}_{1}, \tilde{b}_{2}\right)$ \\
$\quad$ staus & $\left(\tilde{\tau}_{R}, \tilde{\tau}_{L}\right)$ & $\left(\tilde{\tau}_{1}, \tilde{\tau}_{2}\right)$ \\
bósons de Higgs & $H_{u}^{0}, H_{d}^{0}, H_{u}^{+}, H_{d}^{-}$ & $h, H^{0}, A^{0}, H^{ \pm}$ \\
\hline
\end{tabular}

Tabela 2.2: Origem dos estados normalizados para os neutralinos, charginos e stops/sbottoms.

Retomando o problema de ajuste fino, vemos que a introdução dos parceiros supersimétricos traz uma solução para as divergências quadráticas que observamos na seção 2.1. Na Fig. 2.2. colocamos os principais diagramas de supersimetria com contribuição quadrática $\Lambda_{U V}^{2}$ para a massa do bóson de Higgs. Esse diagrama resulta em,

$$
\delta m_{h}=+\frac{3}{8 \pi^{2}} \lambda_{t}^{2} \Lambda_{U V}^{2}-\frac{g^{2}}{16 \pi^{2}} \Lambda_{U V^{2}}-\frac{\lambda^{2}}{16 \pi^{2}} \Lambda_{U V}^{2}+\frac{3}{16 \pi^{2}} m_{\tilde{t}}^{2} \lambda_{t}^{2} \log \frac{\Lambda_{U V}}{m_{\tilde{t}}}+\cdots
$$

Aqui fizemos uma identificação do acoplamento quártico do Higgs do MP e o acoplamento quártico introduzido pelo potencial de quebra soft de supersimetria para os 2 dubletos de Higgs, $H_{u}$ e $H_{d}$.

Comparando com as equações 2.1-2.3), vemos que os diagramas supersimétricos possuem loops com partículas de spin diferentes dos loops do MP, Fig. 2.1, porém supersimetria mantém o vértice que compõe o loop com a mesma dependência de parâmetros do Modelo Padrão, pois partículas de mesmo números quânticos gauge são agrupadas nos mesmos supermultipletos. Isso 
traz o cancelamento exato das divergências quadráticas $\left(\Lambda_{U V}^{2}\right)$ para a massa do bóson de Higgs [35]. Além disso, esse mecanismo de cancelamento garante que todas as outras contribuições, de qualquer férmion do Modelo Padrão, para a massa do bóson de Higgs, também serão canceladas pelos seus parceiros supersimétricos.
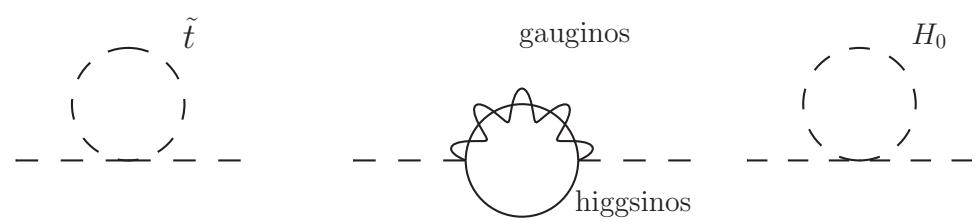

Figura 2.2: Diagramas em 1-loop que cancelam as divergências quadráticas para a massa do bóson de Higgs devido à contribuições mais relevantes vindas do MP. O terceiro diagrama referese ao bóson de Higgs neutro pesado $\left(H_{0}\right)$ introduzido por supersimetria. O segundo e terceiro diagramas dependem do potencial de quebra soft.

Já que supersimetria não é uma simetria observada experimentalmente, ao menos, na escala $\mathrm{TeV}$, temos que introduzir um termos de quebra na lagrangiana supersimétrica. Isso é feito através de um potencial de quebra soft, nele são introduzido termos com acoplamentos de dimensão de massa positiva. São introduzidos termos como massas para gauginos e escalares.

\subsubsection{Paridade-R}

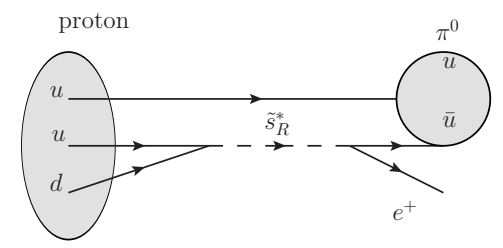

Figura 2.3: Decaimento do próton devido à interações supersimétricas que violam número bariônico e leptônico.

Supersimetria pode introduzir interações que violam número bariônico e leptônico e ainda assim preservar as transformações supersimétricas. Por isso o MSSM necessita de um ingrediente a mais que a supersimetrização somente não fornece. Um mecanismo que proíba ou restrinja fortemente o decaimento do próton [36], através de processos como o ilustrado na Fig. 2.3, onde acoplamentos que violam o número bariônico e leptônico permitem esse fenômeno. Além do processo listado na Fig. 2.3, outros podem acontecer como $p \rightarrow \pi^{0} \mu, \nu \pi^{+}$, etc.

Um modo de contornar esse problema para o MSSM é introduzir uma simetria discreta, a paridade-R. Ela define um número quântico para as partículas do Modelo Padrão igual a +1 e um número quântico para partículas introduzidas por supersimetria igual a -1. Esse número deve ser conservado multiplicativamente em todas as interações do MSSM. A paridade-R proíbe, assim, todas as interações que produzem o decaimento do próton. Vale ressaltar, contudo, que um setor de quebra de paridade-R pode ainda ser acomodado desde que tais acoplamentos sejam muito suprimidos de modo a fazer a vida média do próton tão grande quanto a idade do Universo, pelo menos. Para uma partícula de número bariônico $B$, número leptônico $L$ e spin $s$, a paridade-R será dada por:

$$
P_{R}=(-1)^{(3(B-L)+2 s)} .
$$

Para um colisor do tipo do LHC onde são realizadas colisões entre prótons, temos paridade-R inicial igual a +1 no estado inicial. Isso implica que todas as partículas supersimétricas serão 
produzidas aos pares e no decaimento dessas partículas outra partícula supersimétrica deverá ser produzida. Consequentemente, a partícula mais leve introduzida por supersimetria será estável. Tal partícula massiva, neutra e fracamente interagente (uma LSP, portanto) é uma candidata à matéria escura.

Nos modelos que iremos estudar, o neutralino mais leve $\tilde{\chi}_{1}$ é a LSP, o candidato à matéria escura para o MSSM. No âmbito desse modelo, contudo, ela não é a única candidata viável. Em certos modelos de quebra soft de supersimetria, o gravitino ou sneutrino mais leve podem fazer o papel de matéria escura [2].

\subsubsection{Quebra de supersimetria}

A supersimetria não pode ser uma simetria exata da natureza, pois como vimos no supermultipleto, todas as partículas possuem as mesmas massas. Assim, para o elétron, por exemplo, teríamos o selétrons - escalares de massa $0.5 \mathrm{MeV}$ assim como o elétron. Porém não temos evidências desse tipo de partículas. Isso denota que supersimetria foi quebrada em alguma escala, e por isso, as partículas supersimétricas têm massas muito diferentes de seus parceiros do Modelo Padrão. Chamaremos a escala de quebra de supersimetria de $\Lambda_{\mathrm{SOFT}}$.

Existem diversos mecanismos de quebra de supersimetria que podem ser aplicados ao MSSM, a maioria deles assume que exista um setor em altas energias, puramente supersimétrico, onde a quebra na escala $\mathrm{TeV}$ seja transmitida por alguma interação que tem origem nesse setor. Dentre esses modelos os mais famosos são o mSugra ou CMSSM (minimal supergravity mediation susy breaking) [2, 37] e a mediação gauge GMSSB (gauge mediation susy breaking) [38].

Quando a quebra de supersimetria ocorre, as partículas supersimétricas têm massas muito diferentes dos seus parceiros supersimétricos. Em geral a massa das partículas supersimétricas dependem da escala de quebra $\Lambda_{\text {SOFT }}$. Para o mSugra $\Lambda_{\text {SOFT }}=M_{\text {Planck }}$, para ao GMSSB $\Lambda_{\mathrm{SOFT}}=M_{\text {Mess }}$, onde $M_{\text {Mess }}$ é a massa dos campos de gauge mensageiros.

No mSugra define-se um esquema de quebra de supersimetria e reduz-se os parâmetros livres do MSSM $(\mathcal{O}(100))$ para apenas 5. Os parâmetros livres, no mSugra, são: acoplamento universal trilinear de escalares $\left(A_{0}\right)$, a razão do valor esperado de vácuos dos dois bósons de Higgs $H_{u}$ e $H_{d}(\tan \beta)$, o sinal do parâmetros de massa do Higgs no superpotencial $(\mu)$, a massa universal do escalares $m_{0}$ e a massa universal os gauginos $m_{\frac{1}{2}}$. Nesse modelo, em sua concepção mínima, ou seja, somente com 2 dubletos de bósons de Higgs, resolve o problema de ajuste fino, desde que a escala de quebra soft não seja muito alta. Atualmente, devido à ausência de eventos de supersimetria nos últimos resultados do LHC 7 e $8 \mathrm{TeV}$, as massas das partículas supersimétricas têm limites na faixa de $\sim 1.8 \mathrm{TeV}$, retornando assim com uma certa quantidade de ajuste fino. Conclusões análogas podem ser obtidas para o modelo GMSSB.

Retomando a correção para a massa do bóson de Higgs, em 1-loop, calculada para o Modelo Padrão (2.1) e para supersimetria (2.6), temos que a contribuição total 1-loop será [2]:

$$
\delta m_{h}^{2} \sim \frac{1}{2} m_{\tilde{t}}^{2} \lambda_{t}^{2} \log \frac{\Lambda_{\mathrm{SOFT}}}{m_{\tilde{t}}}+\cdots .
$$

Vemos que a correção total para a massa do bóson de Higgs é livre de divergências quadráticas. Porém para manter a massa do Higgs em $126 \mathrm{GeV}$, com stops muito pesados, introduz-se novamente outro problema de ajuste fino [2].

Existem modelos simplificados que não assumem nenhum esquema de quebra, e logo, não tem seu comportamento em altas escalas definido por equações do grupo de renormalização. Um deles é o pMSSM (phenomenological minimal supersymmetric standard model) [39, 40, 41, onde evidências experimentais como física de sabores (flavor physics) e momento de dipolo para léptons ajudam a restringir os parâmetros introduzidos por supersimetria. Após essas restrições, restam 20 parâmetros livres caso deseje-se fazer o gravitino como LSP, ou 19 se assumirmos que a LSP seja o neutralino [4]. 


\subsubsection{Lagrangiana supersimétrica}

Em nossa análise estamos interessados em processos de produção de squarks e gluinos com posterior decaimento em jatos e MET no colisor próton-próton LHC. Sendo assim, iremos fazer uma descrição somente do setor responsável por essas interações, tornando assim, os modelos analisados nesse trabalho, simplificados. Os termos da lagrangiana relevantes para produção de squarks e gluinos são os da QCD supersimétrica, e o decaimento em jatos e MET é governado por um termo eletro-fraco da lagrangiana supersimétrica [3].

$$
\begin{gathered}
\mathcal{L}_{\mathrm{SUSY}}=\mathcal{L}_{g \tilde{q} \tilde{q}}+\mathcal{L}_{g \tilde{g} \tilde{g}}+\mathcal{L}_{g g \tilde{q} \tilde{q}}+\mathcal{L}_{q \tilde{q} \tilde{g}}+\mathcal{L}_{q \tilde{q} \tilde{\chi}} \tilde{L}_{1}+\text { h.c. } \\
\mathcal{L}_{g \tilde{q} \tilde{q}}=-i g_{s} \sum_{q}\left[\tilde{q}_{L}^{*} \overleftrightarrow{\partial_{\mu}} \tilde{q}_{L}+\tilde{q}_{R}^{*} \overleftrightarrow{\partial_{\mu}} \tilde{q}_{R}\right] T^{a} g^{a \mu}, \\
\mathcal{L}_{g \tilde{g} \tilde{g}}=i \frac{1}{2} g_{s} f^{a b c} \overline{\tilde{g}}^{a} \gamma_{\mu} \tilde{g}^{b} g^{c \mu}, \\
\mathcal{L}_{g g \tilde{q} \tilde{q}}=g_{s} \sum_{q}\left[\tilde{q}_{L \mathbf{i}}^{*} \tilde{q}_{L \mathbf{j}}+\tilde{q}_{R \mathbf{i}}^{*} \tilde{q}_{R \mathbf{j}}\right]\left(T^{a} T^{b}\right)_{\mathbf{i j}} g^{a \mu} g_{\mu}^{b}, \\
\mathcal{L}_{q \tilde{q} \tilde{g}}=-\sqrt{2} g_{s} \sum_{q}\left[\bar{q} P_{R} T^{a} \tilde{g}^{a} \tilde{q}_{L}-\bar{q} P_{L} T^{a} \tilde{g}^{a} \tilde{q}_{R}\right] .
\end{gathered}
$$

Essas lagrangianas são compostas basicamente por squarks Left e Right, os campos $\tilde{q}_{L, R}$, parceiros supersimétricos dos quarks, os campos espinoriais de 4 componentes $q$. Onde $q=(u, d, s, c)$. Temos os glúons, $g^{a}$, que manifestam-se em 8 estados diferentes devido à representação adjunta de $S U(3)_{C}$. Os gluínos são os parceiros supersimétricos dos glúons, denotados pelos espinores de Majorana $\tilde{g}^{a}$. Veja o apêndice A.2

Note que as equações (2.9) não dependem de nenhum parâmetro do modelo supersimétrico e tão pouco do espectro de massas dos squarks e gluinos. Porém o termo $\mathcal{L}_{q \tilde{q} \tilde{\chi}} \tilde{x}_{1}$ depende do modelo de quebra de supersimetria e portanto do potencial de quebra soft [2, 3],

$$
\mathcal{L}_{q \tilde{q} \tilde{\chi}_{1}}=-\frac{g}{\sqrt{2}} \overline{\tilde{\chi}}_{1}^{0}\left[\left(l_{q}^{L} P_{L}+r_{q}^{L} P_{R}\right) \tilde{q}_{L}^{*}+\left(l_{q}^{R} P_{L}+r_{q}^{R} P_{R}\right) \tilde{q}_{R}^{*}\right] q
$$

Aqui as constantes $l$ e $r$ dependem do superpotencial de quebra soft. Portanto são dependentes de modelos. Escolhemos o mSugra pois existem implementações diversas para a produção do espectro supersimétrico como SPheno [42] e SoftSusy [43], adotamos um modelo onde o branching ratio de gluinos em squarks e quarks é de $100 \%$ com squarks Right decaindo em $99 \%$ dos casos em jatos e MET, enquanto que os squarks Left decaem em jatos e MET em 1\% dos casos. Espectros que não têm esse tipo de branching ratio podem levar a estados intermediários de produção de neutralinos pesados e/ou charginos, descaracterizando assim, a produção de eventos de jatos e MET.

A lagrangiana (2.9) deve ser analisada em dois cenário de hierarquia de massas para squarks e gluinos, $m_{\tilde{g}}>m_{\tilde{q}}$ e $m_{\tilde{g}}<m_{\tilde{q}}$. Na região $m_{\tilde{g}}>m_{\tilde{q}}$, os gluinos decaem preferencialmente em squarks e jatos, onde os squarks têm sabores $u, d, s, c$. Para a região do espectro onde $m_{\tilde{g}}<m_{\tilde{q}}$, ainda teremos squarks decaindo em jatos e neutralino, mas os gluinos agora terão seu decaimento em squarks basicamente fora da camada de massa. Isso fará com que os gluinos decaiam em stops e top, pois os stops em geral são os squarks mais leves em modelos com quebra em altas energias [2], assim como no modelo simplificado que utilizamos.

$$
\tilde{g} \rightarrow(\tilde{t})^{*} t \rightarrow\left(\tilde{t}^{*} \rightarrow b \chi_{1}^{ \pm}\right) t,
$$


$\mathrm{Ou}$

$$
\tilde{g} \rightarrow(\tilde{t})^{*} t \rightarrow\left(\tilde{t}^{*} \rightarrow t \chi_{1,2,3}^{0}\right) t
$$

Em geral os charginos decaem em bósons $W$ e os neutralinos pesados em bósons $Z$, sempre acompanhados de partículas supersimétricas mais leves, até produzir o neutralino leve $\left(\tilde{\chi}_{1}\right.$, matéria escura). A produção de quarks top e bottoms está fora dos eventos de jatos e MET, pois pode-se realizar, com uma eficiência razoável, o tagging dos jatos produzidos por essas partículas, e identificar esses jatos. Esses decaimentos não são contabilizados como válidos após seleção de eventos em nossa análise e por isso foram desconsiderados das simulações, com isso, somente a produção de squarks é relevante para a região $m_{\tilde{g}}<m_{\tilde{q}}$.

\subsubsection{Limites para supersimetria}

Experimentos para detecção de supersimetria já são realizados há algum tempo. Dentre os mais conhecidos temos o colisor LEP (Large Electron-Positron) no CERN e o Tevatron, um colisor do tipo próton-antipróton localizado no Fermilab e o LHC large hadron collider localizado no CERN. Além de outros experimentos para detecção indireta e investigações em fenômenos de baixa energia. Em geral esses experimentos são analisados em contextos de modelos simplificados e no CMSSM. A seguir temos uma breve exposição e comentários sobre esses experimentos:

\section{Procura em Colisores}

O colisor Tevatron concentrou-se na procura de supersimetria para eventos com jatos e MET. O experimento CDF e D0 localizados no Tevatron colocaram limites para a massa de squarks e gluinos em $400 \mathrm{GeV}$ para o CMSSM [45, 46]. Já no LEP o experimento DELPHI realizou buscas para gauginos e sléptons também no CMSSM [47], onde obtiveram limites para o neutralino mais leve e charginos de $45.5 \mathrm{GeV}$ e $94 \mathrm{GeV}$, respectivamente. O limite imposto para os sléptons também teve contribuições do experimento ALEPH, com isso alcançou-se restrições para massa de sléptons de até $100 \mathrm{GeV}$ [48]. Nos três anos de funcionamento do LHC muitos limites foram impostos para modelos supersimétricos como o CMSSM. A maioria dos limites foram impostos através de medidas realizadas para procura de stops, sbottoms e jatos + MET. Na Fig. 2.4 vemos os resultados recentes obtidos pelo CMS [49]. Vemos limites para a massa de gluinos e para squarks em função das massas do neutralino, em uma realizada para procura de eventos com jatos e MET para modelos simplificados de supersimetria. Limites da ordem de $1.0 \mathrm{TeV}$ são impostos para squarks e $1.3 \mathrm{TeV}$ para gluinos. Com base nesses dados construímos a nossa análise. Nesses valores de massas análises simplificadas deixam de ser eficientes devido aos grandes backgrounds associados, por isso uma análise multivariada passa a ter um lugar de destaque. Ressaltamos que na Fig. 2.4a limites para massas de neutralinos acima de $500 \mathrm{GeV}$, praticamente não existem.

\section{Procura em Astrofísica}

A medida da densidade de relíquia contribui para adequar o MSSM para um modelo mais realista. Após a expansão do Universo a níveis adequados, a densidade de matéria escura torna-se baixa, devido à sua baixa seção de choque acabamos em um cenário onde essa densidade de matéria escura torna-se praticamente estável. Essa densidade é conhecida como densidade de relíquia, e foi medida pelo WMAP em 2011 [24. O MSSM tem o neutralino como candidato à matéria escura quando introduzimos paridade-R, porém nem todos os modelos de quebra de supersimetria para o MSSM resultam em densidades de relíquia que observamos hoje. Com isso podemos restringir diversos valores de parâmetros livres para o CMSSM.

\section{Procura em Baixas Energias}

Em baixas energias pode-se restringir supersimetria através de medidas indiretas. Uma 


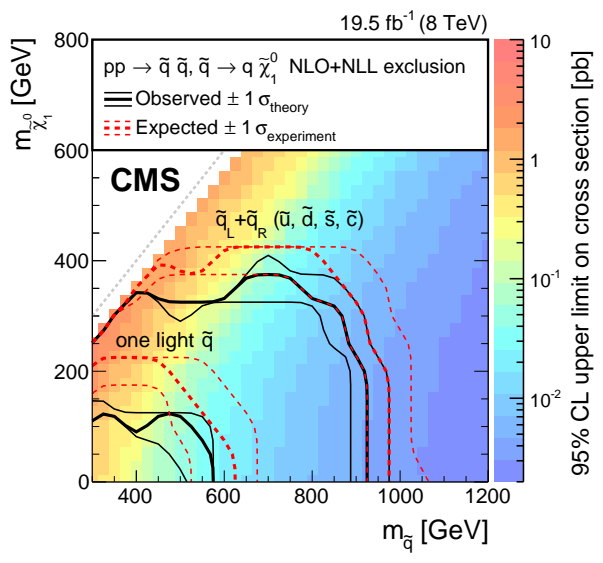

(a)

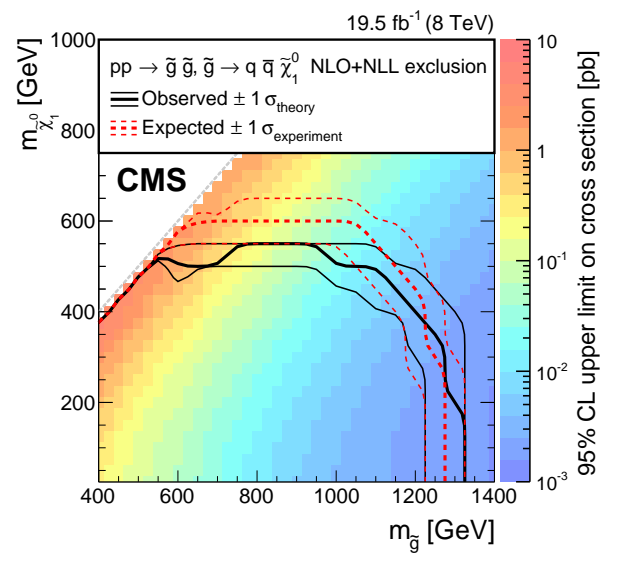

(b)

Figura 2.4: Resultados para o LHC $8 \mathrm{TeV}$ dados pelo CMS. Limites para gluinos de até 1.3 $\mathrm{TeV}$ podem ser alcançados em uma análise com quarks tops e MET no estado final. Já para os squarks o limite é de no máximo $1 \mathrm{TeV}$. Neutralinos com mais de $500 \mathrm{GeV}$ ainda não possuem limites estabelecidos. Análise feita para modelos simplificados de supersimetria. Fonte: [49]

delas é o processo $b \rightarrow \gamma s$. Esse processo envolve diagramas em 1-loop para partículas pesadas supersimétricas. Assim não observar os efeitos desse loop impõe fortes limites para as massas dessas partículas [50]. Também pode-se restringir supersimetria em medições do momento magnético do múon $(g-2)$, novamente devido à contribuições de loops de partículas pesadas [51].

\section{$2.3 \quad$ Massas de squarks e gluinos}

Modelos como o mSugra ou GMSSB possuem um esquema de quebra em altas energias, isso proporciona um pequeno número de parâmetros livres que definem todo o modelo. Esses esquemas de quebra de supersimetria necessitam de diversas condições que têm validade somente no setor de altas energias. Esses setores são conhecidos como hidden sectors ou setores escondidos, assume-se ainda, que esses setores são totalmente supersimétricos. Com isso todos os parâmetros são definidos em altas energias, conhecidas como escalas mediadoras $(M)$. Por exemplo, no mSugra temos $M=M_{\text {Planck }}$ e no modelo de mediação via bósons de gauge GMSSB $M=$ $M_{\text {Mess }}$. Porém para definirmos a teoria na escala TeV precisamos usar as equações do grupo de renormalização e converter esses parâmetros de altas energias para baixas energias.

No MSSM os squarks recebem correções quânticas em suas massas através de, basicamente, gluinos em 1-loop em altas energias.

$$
m_{\tilde{q}}^{2}(Q) \sim m_{\tilde{q}}^{2}(M)+A_{\tilde{q}}(M) m_{\tilde{q}}^{2}+A_{\tilde{g}}(M) m_{\tilde{g}}^{2}
$$

onde $Q \sim \mathcal{O}(T e V)$ e $M \sim \mathcal{O}\left(M_{G U T}=10^{16}\right)$. Acoplamentos de Yukawas foram desconsiderados e $A_{\tilde{g}}$ domina sob $A_{\tilde{q}}$, devido à grande multiplicidade dos gluinos [52].

Já os gluinos não recebem correções em 1-loop vindas de squarks, pois a simetria quiral protege as massas dos gluinos em qualquer ordem de teoria de perturbação, assim como a simetria gauge protege as massas dos bósons de gauge. Portanto para gluinos temos,

$$
m_{\tilde{g}}^{2}(Q) \sim m_{\tilde{g}}^{2}(M)+B_{\tilde{g}}(M) m_{\tilde{g}}^{2} .
$$

Por não haver uma simetria desse tipo para escalares temos o problema de hierarquia. Em suma, a massa do squarks não pode ser feita muito mais leve do que as massas dos gluinos. 


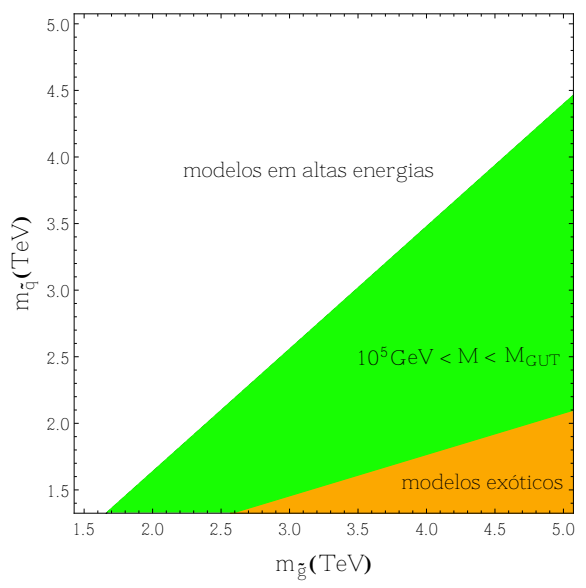

Figura 2.5: Região do plano $\left(m_{\tilde{q}}, m_{\tilde{g}}\right)$ analisada em nosso trabalho. Vemos que algumas regiões são favorecidas por modelos com quebra de supersimetria em altas energias enquanto outras não.

Na Fig. 2.5 temos uma ilustração da relação de massa entre squarks e gluinos em diferentes cenários [52]. A região superior denotada por "modelos em altas energias" delimita os modelo com quebra em altas energias como o mSugra ou GMSSB. A região intermediária, é acessível somente a modelos com quebra em escalas intermediárias. Aqui o mSugra, por exemplo, está excluído. Já a região "modelos exóticos" permite apenas modelos com uma diferença pequena entre a escala de mediação de quebra e a escala eletro-fraca, podem ser modelos com supersimetria estendida. Em nosso estudo, pontos ao longo de todo este espaço de massas foram considerados dentre os 150 espectros simulados.

Nos modelos simplificados a lagrangiana é determinada em teorias efetivas para a escala específica do fenômeno, em nosso caso a escala do colisor LHC, $14 \mathrm{TeV}$. Nesses modelos nenhuma equação do grupo de renormalização são consideradas, portanto as massas e larguras são definidas como parâmetros livres.

\subsection{Dimensões extras universais mínimas}

Modelos com dimensões extras podem ser definidos em contextos onde a gravidade é a única interação que propaga-se pela dimensão extra ou onde todas as partículas do Modelo Padrão e a gravidade propagam-se pela dimensão extra [4], neste último caso, dizemos que ela é universal.

Para modelo com uma dimensão extra compacta, assume-se que além das 4 dimensões que vivemos, existem uma dimensão extra compacta de raio $R$, a dimensão extra pode estar contida em $[0,2 \pi R]$ e seus pontos extremos são identificados. Todos os campos podem propagar-se pela dimensão extra. A lagrangiana para esse modelo é construída em um contexto de teoria de campos em 5 dimensões $(D=5$ ou $5 \mathrm{D})$, e por isso têm acoplamentos 5 -dimensionais com dimensão de massa negativa, e portanto, não renormalizáveis. Como a maioria das teorias nãorenormalizáveis, essa teoria tem validade limitada até um certa escala $\Lambda_{\mathrm{DE}}$.

Como o espaço é expandido para 5 dimensões, deve-se definir momento e posição na quinta dimensão extra compacta:

$$
\begin{aligned}
& x^{M}=\left(x^{\mu}, y\right), \\
& p^{M}=\left(p^{\mu}, p^{5}\right) .
\end{aligned}
$$

A métrica desse espaço é do tipo Minkowski para os índices espaço-tempo $\mu$ e plana para a dimensão extra compacta y. Porém os campos definidos nesse espaço devem obedecer condições periódicas na dimensão extra compacta, levando assim, à quantização do momento na dimensão extra, $p^{5}= \pm \frac{n}{R}$. Onde $n$ é um número inteiro e conhecido como número-KK. O invariante 
multi-dimensional $p_{M} p^{M}=m^{2}$ é igual a massa de uma dada partícula, com isso temos:

$$
p^{2}=p_{\mu} p^{\mu}=m^{2}+\frac{n^{2}}{R^{2}} .
$$

Vemos que em $\mathrm{D}=5$, onde temos 1 dimensão extra compacta, temos uma torre infinita de partículas, cada uma com massa proporcional à massa do modo zero $(n=0)$ e ao raio de compactificação $R$. Esse resultado denota que um campo em $\mathrm{D}=5$, quando expandido em nosso espaço 4-dimensional, leva à uma torre infinita de campos, essa torre é conhecida como torre de Kaluza-Klein. O momento $p^{M}$ é um quantidade conservada na dimensão extras compacta de raio $R$.

O modo zero dos campos $(n=0)$ representam as partículas que observamos no Modelo Padrão. Para $n>0$ começamos a ter outros campos mais pesados, chamamos de estados excitados, esses campos possuem todos os números quânticos idênticos ao respectivo modo zero. Por exemplo, um quark terá estados excitados com mesmo spin, hiper-carga, quiralidades, etc, porém com massas diferentes. Além disso os campos vetoriais terão uma componente extra na quinta dimensão, denotada por uma partícula extra, teremos tantos campos quanto partículas vetoriais no Modelo Padrão.

Teremos que lidar com os seguintes problemas: primeiro, refere-se às projeções de quiralidade para os férmions, sabemos que a interação eletro-fraca distingue quiralidade, portanto deve-se encontrar um meio de escrever férmions com modo zero Righ ou Left. O segundo refere-se às componentes extras da quinta dimensão para os bósons de gauge $\left(\left(A_{5}, A_{\mu}\right)\right)$, elas trazem novas partículas em $\mathrm{D}=4 \mathrm{com}$ modos zero massivos, veja apêndice A.3. Contornando esses problemas poderemos escrever o Modelo Padrão em uma teoria de dimensão extras, em nosso caso, estaremos interessados em uma teoria onde todos os campos podem se propagar livremente pela única dimensão extra compacta, tal modelo é conhecido como dimensão extra universal mínima, ou em inglês, MUED (minimal universal extra dimension). O termo mínimo vem do fato de considerar apenas uma dimensão extra compacta, podemos construir modelos com duas dimensões extras compactas veja [4], esses modelos são conhecidos somente como UED.

Para lidar com esses dois problemas utiliza-se a compactificação no orbifold, Fig. 2.6 [53].

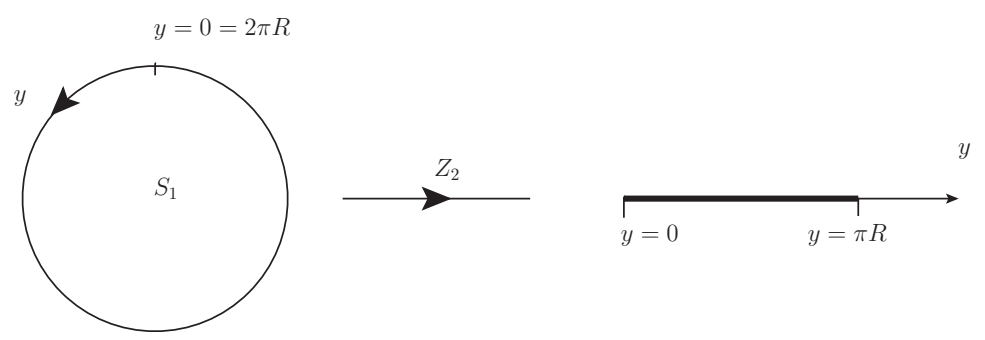

Figura 2.6: Esquema da compactificação no orbifold $\left(S_{1} / Z_{2}\right)$.

Inclui-se uma paridade para a dimensão extra, reduzindo assim seu tamanho. Essa identificação pode ser resumida na operação $y \rightarrow-y$ e chamamos de operação $Z_{2}$. A dimensão extra agora está contida $[0, \pi R]$. A lagrangiana de MUED deve ser invariante sob $Z_{2}$. Os campos assumem um propriedade interessante após essa compactificação no orbifold. Campos que são pares sob $Z_{2}$ têm modo zero, enquanto que campos ímpares sob $Z_{2}$ não têm modo zero. Isso resolve o problema de quiralidade em teoria eletro-fraca para os férmions, utilizando condições de contornos apropriadas podemos definir campos com modo zero Righ ou Left. Além disso as projeções dos campos vetoriais na quinta dimensão passam a ter paridade ímpar sob $Z_{2}$, garantindo que seus modos zero desapareçam, veja apêndice A.3. Ressaltamos que os campos em 5 dimensões não podem misturar estados pares e ímpares sob $Z_{2}$.

Os estados excitados $(n>0)$, após compactificação, terão todos acoplamentos vetoriais. Ou seja, somente os modos zero irão distinguir as interações eletro-fracas. 
Aparentemente as partículas em MUED tendem a ser altamente degeneradas em massa a nível de árvore, veja 2.21, se $R^{-1}>>m$ os estados excitados são praticamente degenerados. Porém correções em 1-loop tendem a dar grandes contribuições para as massas dos estados excitados, quebrando essa degenerescência [5].

\subsubsection{Paridade-KK}

A compactificação no orbifold $Z_{2}$ quebra a simetria de translação na dimensão extra. Assim o momento $p^{5}$ não será mais conservado e consequentemente, o número-KK também deixa de ser conservado.

Quando as interações ocorrem em altas energias $q^{2}>>\left(\frac{1}{R}\right)^{2}$, não há possibilidade de identificar os pontos fixos $y=0$ e $y=\pi R$, retornando aparentemente ao caso onde não existia compactificação no orbifold. Com isso o número-KK volta a ser conservado. Isso denota que a violação do número-KK pode estar concentrada nos pontos fixos. Quando escrevemos a lagrangiana nesses pontos vemos que deve existir uma paridade para cada uma das partículas da lagrangiana de MUED [4, 6]. Essa é a paridade-KK, e é dada por:

$$
P_{K K}=(-1)^{n}
$$

onde $n$ é o número-KK. Na Fig. 2.7 temos quatro exemplos da aplicação da paridade-KK. Observe que no terceiro decaimento o número-KK, e portanto o momento na quinta dimensão, não é conservado (partículas no orbifold), porém a paridade-KK é conservada. Além disso, a paridade-KK é conservada sob correções quânticas. O mesmo não pode ser dito para o númeroKK.
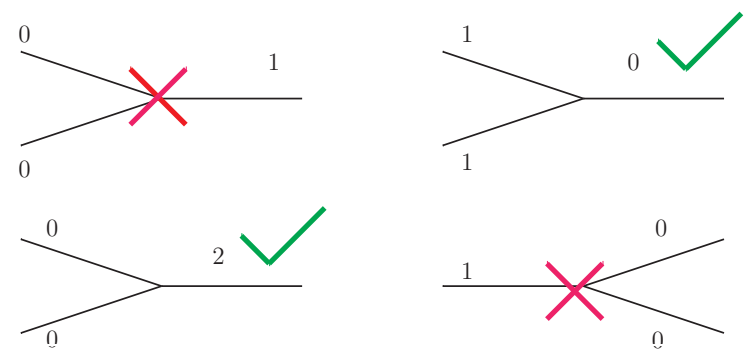

Figura 2.7: Aplicação da paridade-KK. Os números indicam o número-KK de cada uma das partículas dos vértices.

As consequências da paridade-KK são, fenomenologicamente, idênticas à paridade-R em supersimetria. A partícula mais leve dos estados excitados, neutra, massiva e portanto um candidato à matéria escura é o estado correspondente ao quanta do grupo $U(1)_{Y}: B^{(1)}$ - o primeiro modo KK do fóton (fóton pesado).

Os estados com número-kk igual a 1 possuem topologia muito parecida com as de supersimetria após a compactificação no orbifold. Por isso o modelo de dimensões extras universais é tão utilizado em comparações com modelos de supersimetria. Pois ambos podem produzir estados finais com jatos ou léptons e grande quantidade de MET em diversos fenômenos diferentes, como discutimos anteriormente.

\subsubsection{Limites para MUED e massas de KK-quarks e KK-glúons}

Para o modelo de MUED também é possível impor limites para o raio de compactificação através de mediadas de precisão, como o momento magnético anômalo do múon e o branching ratio de alguns decaimentos raros, como o $b \rightarrow s \gamma$ [54].

A densidade de relíquia também pode ser usada para estimar o valor da massa do KK-fóton assim impor limites para o modelo de MUED. Como o espectro de MUED é mais degenerado 
do que o supersimétrico, muitos processos participam da co-aniquilação até a produção da matéria escura. Foi mostrado, veja [60], que introduzir processos de co-aniquilação, em geral, leva ao aumento da densidade de relíquia, consequentemente, diminuindo a massa da partícula de matéria escura. Isso coloca o KK-fóton com limites de massas maiores do que o neutralino de supersimetria.

Em colisores, no Tevatron por exemplo, a busca ocorreu com eventos de dois léptons de mesma carga [61], nesse contexto modelos gerais de dimensões extras eram analisados, no qual o modelo de MUED é um caso particular. Também foram analisados eventos com dois fótons no estado final e MET, nesses modelos uma LKP pode decair através de interação gravitacional em um fóton e um KK-gráviton 62. No LHC o experimento ATLAS [63] realizou uma busca de eventos com léptons de cargas opostas no estado final. É possível que tal assinatura possa vir de um decaimento direto de um KK-fóton ou KK-bóson Z. No caso de MUED, o decaimento do fóton pesado é proibido, porém o decaimento do KK-bóson $\mathrm{Z}$ pode dar origem a esse tipo de assinatura, limites para massa desse bóson estão entre 3.0 e 4.0 TeV. Não há buscas específicas no modelo de MUED no ATLAS e CMS, e os limites para o raio de compactificação são dados basicamente por físicos teóricos. O limite para $R^{-1}$ é de $1.3 \mathrm{TeV}$ com cut-off ultravioleta de 10 $\mathrm{TeV}$ [60, 64, 65]. Esses limites são suficientes para produzir KK-quarks e KK-glúons com massas da ordem que estamos analisando nesse trabalho, porém um KK-fóton muito pesado $\mathcal{O}(\mathrm{TeV})$ não é privilegiado pelas observações de densidade de relíquia, para modelos típicos de MUED.

Sem considerar termos localizados na fronteira do orbifold, o espectro típico de MUED é altamente degenerado. A massa do $n$-ésimo estado excitado de Kaluza-Klein correspondente a uma partícula do MP de massa $m_{0}$ pode ser obtida através de 2.21), será:

$$
m_{n}^{2}=m_{0}^{2}+\frac{n^{2}}{R^{2}} .
$$

Contudo, correções radiativas devida aos termos cinéticos dos campos do MP localizados na fronteira do orbifold quebram essa degenerescência severa [59]. Ainda assim, em comparação aos espectros típicos de SUSY, os de MUED são muito degenerados. Por exemplo, se $R^{-1}=500$ $\mathrm{GeV}$ é a escala $\Lambda$ onde estes termos cinéticos são importantes, tal que $\Lambda R=20$, a diferença de massa entre o primeiro estado excitado do glúon e do fóton pesado é de apenas $150 \mathrm{GeV}$ [59].

\subsubsection{Lagrangiana de MUED}

Iremos escrever a lagrangiana do primeiro estado excitado $(n=1)$, ou seja, os KK-quarks, kk-glúons e KK-fótons. Note que temos dois quarks excitados, os quarks dubletos $Q^{(1)}$ e quarks singletos $q^{(1)}$. No apêndice A.3 temos as ferramentas necessárias para reproduzir a lagrangiana usada nesse trabalho para MUED. Os termos relevantes para a interação do Modelo Padrão e as partículas do primeiro estado KK, para a nossa análise de jatos e MET, são:

$$
\begin{gathered}
\mathcal{L}_{\mathrm{KK}}=\mathcal{L}_{g q^{(1)} q^{(1)}}+\mathcal{L}_{g g^{(1)} g^{(1)}}+\mathcal{L}_{q q^{(1)} g^{(1)}}+\mathcal{L}_{q q^{(1)} B^{(1)}}+\text { h.c. }, \\
\mathcal{L}_{g q^{(1)} q^{(1)}}=-g_{s} \bar{Q}_{R, L}^{(1)} \gamma^{\mu} g_{\mu} Q_{R, L}^{(1)}-g_{s} \bar{q}_{R, L}^{(1)} \gamma^{\mu} g_{\mu} q_{R, L}^{(1)}, \\
\mathcal{L}_{q q^{(1)} g^{(1)}}=-g_{s}\left[\bar{q}_{L} \gamma^{\mu} g_{\mu}^{(1)} Q_{L}^{(1)}+\bar{q}_{R} \gamma^{\mu} g_{\mu}^{(1)} q_{R}^{(1)}\right], \\
\mathcal{L}_{g g^{(1)} g^{(1)}}=-\frac{g}{2} f^{a b c}\left[\left(\partial_{\mu} g_{\nu}^{a}-\partial_{\nu} g_{\mu}^{a}\right) g^{(1), b \mu} g^{(1), c \nu}\right. \\
\left.+\left(\partial_{\mu} g_{\nu}^{(1), a}-\partial_{\nu} g_{\mu}^{(1), a}\right) g^{b \mu} g^{(1), c \nu}-\left(\partial_{\mu} g_{\nu}^{(1), b}-\partial_{\nu} g_{\mu}^{(1), b}\right) g^{a \mu} g^{c \nu}\right] .
\end{gathered}
$$


Tipicamente em MUED $\gamma^{(1)} \approx B^{(1)}$, ou seja, o fóton pesado é praticamente dominado pela componente do bóson $B_{\mu}^{(n)}$. Isso decorre do fato de que misturas desses bósons dependem de uma espécie de ângulo de Weinberg para os KK-bósons de gauge, os termos diagonais dependem de $R^{-1} \sim \mathrm{TeV}$, fazendo com que as misturas desapareçam. Continuaremos denotando o fóton pesado por $B^{(1)}$. Finalmente temos,

$$
\mathcal{L}_{q q^{(1)} B^{(1)}}=\mathcal{L}_{q q^{(1)} B^{(1)}}=-g\left[y \bar{q}_{L} \gamma^{\mu} B_{\mu}^{(1)} Q_{L}^{(1)}+y^{\prime} \bar{q}_{R} \gamma^{\mu} B_{\mu}^{(1)} q_{R}^{(1)}\right]+\text { h.c. }
$$

O modelo de MUED usado em nosso trabalho também é inspirado em um modelo simplificado. Onde KK-glúons $g^{(1)}$, KK-quarks $Q^{(1)}, q^{(1)}$ produzem jatos e $B^{(1)}$ através de interações de QCD e eletro-fracas, respectivamente, em um contexto de teoria efetiva em dimensões extras. Assim como no caso de supersimetria, mantendo as ressalvas feitas na seção anterior, temos uma certa liberdade para definição das massas do modelo de MUED.

\subsection{Análise estatística}

A análise estatística empregada em nosso trabalho baseia-se em testes de hipóteses construídas através de vários observáveis físicos diferentes. Para o caso de descoberta de nova física definimos a hipótese nula $\left(H_{0}\right)$ representando os backgrounds ou Modelo Padrão e a hipótese alternativa $\left(H_{1}\right)$ representando backgrounds e o sinal de nova física, em nosso caso, supersimetria e Modelo Padrão. Para o discernimento de nova física definimos $H_{0}$ como supersimetria e Modelo Padrão e $H_{1}$ como MUED e Modelo Padrão. Todas essas hipóteses são denominadas simples, pois não existe nenhum parâmetro livre, já que definimos previamente todas as massas, larguras e acoplamentos de cada um dos modelos (hipóteses).

No teste de hipóteses é possível cometer dois tipos de erros, tipo-I $(\alpha)$ e tipo-II $(\beta)$. Esses erros são reportados como probabilidades. No caso onde estamos analisando uma possível descoberta de sinal de nova física, o erro do tipo-I é denominado falso positivo. Ocorre quando o teste afirma que os dados são condizentes com eventos de nova física quando na verdade são, de fato, devidos ao Modelo Padrão. O erro do tipo-II é denominado falso negativo, ocorre quando o teste estatístico afirma que os dados não são explicados por um dado modelo de nova física, quando na realidade são eventos de nova física [55, 56, 57, 58].

O valor do erro tipo-I $(\alpha)$, que deseja-se trabalhar ou alcançar é estipulado a priori. Para física de partículas adota-se $\alpha<2.87 \cdot 10^{-7}$ para definir descoberta de nova física. Esse valor é equivalente a uma probabilidade de se obter significância maior ou igual $5 \sigma$ ao se sortear uma variável aleatória distribuída de acordo com uma Normal Padrão. O valor do erro tipo-II $(\beta)$ é tal que, se $\beta<5 \%$, temos a rejeição da hipótese alternativa. Essas duas condições precisam ser cumpridas, pode-se ter uma medida onde $\alpha=10^{-10}$ mas se o $\beta$ dessa medida cair na região de exclusão, $\beta<5 \%$, dizemos que o teste de hipóteses é inconclusivo. Os possíveis erros do teste de hipóteses $(\alpha$ e $\beta$ ) são escolhidos de acordo com as convenções estipuladas para física de partículas. Veja que são tratados de maneira assimétrica, pois é mais aceitável assumir o risco de uma exclusão por engano de um modelo de nova física qualquer, do que assumir um alto risco de uma falsa descoberta.

Fixado o erro do tipo-I, o teste de hipóteses é sistematizado sempre de forma a minimizar o erro do tipo-II. Podemos definir o melhor teste de hipótese como aquele que maximiza $1-\beta$, o poder do teste (menor chance de falsa rejeição de nova física), para um dado valor fixo de chance de falsa descoberta de nova física.

O teorema de Neyman-Pearson (NP) [17] afirma que o teste estatístico para hipóteses simples que possui maior poder do teste, é aquele definido dentro de uma região dada pela razão de likelihoods $\frac{L\left(\text { resultado } \mid H_{1}\right)}{\left.L \text { (resultado } \mid H_{0}\right)}>k_{\alpha}$, qualquer outra região do mesmo tamanho terá menor poder do teste. $k_{\alpha}$ é definido de acordo com o tamanho do erro do tipo-I desejado. Em nosso caso, por exemplo, define-se $k_{\alpha}$ como a região delimitada por um corte para a estatística-teste, veremos isso mais adiante. 
A ideia por trás da razão de likelihoods reside no fato de que, se nossos dados são melhores descritos pela hipótese nula, então $L\left(\right.$ resultado $\mid H_{0}$ ) será maior do que $L$ (resultado| $H_{1}$ ), o que fornecerá uma razão likelihood pequena, e vice-versa. Assim essa razão comporta-se como um teste para discernir entre uma ou outra hipótese.

Quando incertezas sistemáticas são inseridas nos likelihoods através de marginalizações o teorema de NP, em geral, não é mais válido. Porém, como iremos constatar no próximo capítulo, o teste de hipóteses construído com a razão likelihood, ainda é uma opção muito melhor do que a simples contagem de eventos comumente usada em trabalhos de fenomenologia.

Em física de partículas uma distribuição muito usada é a distribuição de Poisson, pois a população de um determinado bin em um dado histograma, quando os dados são coletados em um intervalo fixo de tempo, é considerada uma variável aleatória que segue distribuições de Poisson. Trata-se de uma distribuição de probabilidade discreta que mensura a probabilidade da ocorrência de $r$ eventos independentes em um intervalo de tempo $t$, onde a taxa de eventos é $\mu$ :

$$
\begin{gathered}
P(r ; \lambda)=\frac{e^{-\lambda} \lambda^{r}}{r !}, \\
\lambda=\mu t .
\end{gathered}
$$

$\lambda$ é o número médio de eventos em um intervalo de tempo $t$.

Observe a Fig.[2.8], nela a distribuição de Poisson tende a uma distribuição gaussiana para valores médios grandes. A partir de $\lambda=20$, ou até valores menores, a concordância com gaussianas é muito boa.

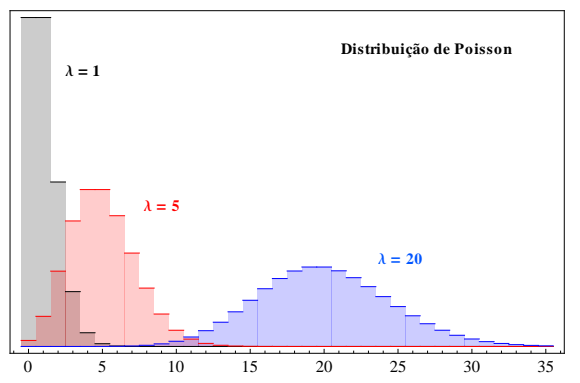

Figura 2.8: Distribuição discreta de Poisson, para valores médios em um dado intervalo fixo de tempo de $\lambda=1, \lambda=5$ e $\lambda=20$.

\subsection{Construção dos histogramas de verossimilhança}

Histogramas de cada uma das distribuições cinemáticas usadas na construção da nossa estatística foram gerados em simulações de Monte Carlo, que oportunamente descreveremos no próximo capítulo. Como vimos na seção anterior, um dado bin $i$, sofre uma flutuação estatística de acordo com uma Poisson de média $\mu_{i}$. O likelihood para uma dada distribuição com $n$ bins de um observável $j$ é calculado como:

$$
L(\vec{N} \mid H)=\prod_{i}^{n} \mathcal{P}\left(N_{i}, \mu_{i}\right),
$$

onde,

$$
\mathcal{P}\left(N_{i} \mid \mu_{i}\right)=\frac{\mu_{i}^{N_{i}} e^{-\mu_{i}}}{N_{i} !} .
$$

$H$ denota algum modelo, em nosso caso pode ser o Modelo Padrão, supersimetria ou MUED. $\overrightarrow{\mu_{j}}=\left(\mu_{1}, \mu_{2}, \ldots, \mu_{n}\right)$ é a população esperada em cada um dos $n$ bins do histograma $j$. Esses 
valores esperados são obtidos através de nossas simulações com geradores de eventos e correspondem às nossa predições teóricas para sinais e backgrounds. Para o histograma $j$, o valor do $i$-ésimo bin é dado por:

$$
\mu_{i}=\sum_{k=1}^{n_{\text {fontes }}} L \sigma_{k} \mathcal{E}_{k i}
$$

$\sigma_{k}$ é a seção de choque total, $L$ a luminosidade integrada e $\mathcal{E}_{k i}$ uma eficiência, que em nosso caso é função do tagging de quarks de jatos, eficiência de cortes e K-factors para correções NLO. $\mathrm{O}$ índice $k$ indica uma soma realizada sobre os processos para um dado modelo (supersimetria, MUED ou Modelo Padrão).

Na equação 2.31, $N_{i}$ é valor flutuado em um dado bin $i$ da distribuição de interesse através da Poisson de parâmetro $\mu_{j i}$. Para cada sorteio de um conjunto de $N_{i}, i=1, \cdots, n$ valores, a partir dos correspondentes $\mu_{j i}$, obtemos um novo histograma. Essa é uma maneira simples de, na verdade, simular o próprio experimento, de criar "dados". É claro que tais "dados" são gerados de acordo com as idiossincrasias de nossas simulações, por isso, os chamamos de pseudoexperimentos.

A partir de agora, iremos denotar a variável $\mu_{i}$ para o Modelo Padrão por $b_{j i}$. Analogamente $\mu_{i}$ será denotada por $s_{j i}\left(u_{j i}\right)$ quando se referir à população do histograma $j$ para o bin $i$ do modelo de supersimetria (MUED). O índice $j$ varia de 1 a 9 , o número de observáveis físicos sensíveis ao spin que escolhemos e que serão apresentadas na próxima seção, e $i$ varia de 1 a 20 , o número de bins de cada histograma. A escolha do número de bins é feita baseada na precisão experimental do ATLAS.

No próximo capítulo iremos abordar a obtenção dos valores de $s_{i j}, u_{i j}$ e $b_{i j}$, que é feita através de geradores de eventos, particularmente o MADGRAPH5.

A probabilidade conjunta, ou verossimilhança, associada ao histograma de uma distribuição cinemática, envolve uma quantidade de informação muito maior do que o simples número total de eventos, ela codifica o próprio formato da distribuição através do vetor $\left(\mu_{1}, \cdots, \mu_{n}\right)$. A ideia pode, agora, ser estendida a todos as distribuições usadas no discernimento. Para $n_{\text {dist }}$ distribuições, cada uma dividida em $n$ bins, o likelihood dos backgrounds é dado por:

$$
L=\prod_{j=1}^{n_{\text {dist }}} \prod_{i=1}^{n} \mathcal{P}\left(N_{j i} \mid b_{j i}\right),
$$

ao passo que o likelihood associado à hipótese de sinal+backgrounds é,

$$
L=\prod_{j=1}^{n_{\text {dist }}} \prod_{i=1}^{n} \mathcal{P}\left(N_{j i} \mid s_{j i}+b_{j i}\right) .
$$

Vamos agora mostrar como combinar estas informações numa única estatística e avaliar sua função de distribuição.

\subsection{Estatística-teste para descoberta e discernimento}

Uma estatística-teste, $\lambda$, é uma função criada a partir de um conjunto de medidas experimentais e de predições teóricas. Construída a estatística-teste e armados de um teste estatístico é possível investigar a concordância de um dado observado com uma determinada hipótese teórica. Suponha que temos um conjunto de dados observados organizados em bins $\vec{o}=\left(o_{1}, \ldots, o_{n}\right)$, ou de pseudo-experimentos simulados de acordo com algum modelo pré-estabelecido.

Nosso objetivo é estimar as PDFs da estatística-teste assumindo, ora a hipótese nula, ora a hipótese alternativa, obtendo $f\left(\lambda(\vec{o}) \mid H_{0}\right)$ e $f\left(\lambda(\vec{o}) \mid H_{1}\right)$, respectivamente. Onde $f$ é uma PDF. 
Escolhemos aqui como estatística-teste a razão dos likelihoods (teorema de NP) associados a cada hipótese, pois testes estatísticos construídos dessa maneira oferecem o melhor resultado para um teste de hipóteses simples, como discutimos anteriormente. Ao invés da razão pura dos likelihoods é comum usar o logaritmo da razão likelihood, pois ele tem a propriedade de converter multiplicações em simples somas e exponenciais em fatores multiplicativos, além do fato de ser uma função monotonamente crescente, assim como a razão likelihood. Isso simplifica computacionalmente os cálculos para construção do teste estatístico. A estatística-teste likelihood ratio torna-se, então, log-likelihood ratio $(\Lambda)$ :

$$
\Lambda=-2 \ln \left(\frac{L\left(N \mid H_{1}\right)}{L\left(N \mid H_{0}\right)}\right)
$$

A primeira análise que vamos fazer é para descoberta de sinal de nova física, ou seja, a hipótese nula será o Modelo Padrão e a alternativa o Modelo Padrão mais supersimetria:

$$
\begin{aligned}
& n_{0 i j}=b_{i j}, \\
& n_{1 i j}=b_{i j}+s_{i j} .
\end{aligned}
$$

Temos condições agora de calcular 2.35 usando 2.34 e 2.31):

$$
\Lambda=\sum_{i=1}^{n_{\text {dist }}} \sum_{j=1}^{n_{b i n s}} 2\left(s_{i j}-r_{i j} \ln \left(1+\frac{s_{i j}}{b_{i j}}\right)\right) .
$$

onde $r_{j i}$ representa o número de eventos observados (ou de um pseudo-experimento) no bin $i$ de uma distribuição $j$. Os valores de $r_{i j}$ simulam os próprios dados do experimento, para isso são sorteados de uma Poisson de média $b_{i j}, s_{i j}+b_{i j}$ ou $u_{i j}+b_{i j}$ dependendo das hipóteses a serem testadas. Estes experimentos fictícios são os pseudo-experimentos pseudo-experimentos.

Após a descoberta de nova física, o próximo passo seria dizer quais modelos explicam melhor os dados vindos de excessos de eventos encontrados. Para a realização desse trabalho definiremos

$$
\begin{aligned}
& n_{0 i j}=b_{i j}+s_{i j}, \\
& n_{1 i j}=b_{i j}+u_{i j} .
\end{aligned}
$$

O variável $u_{i j}$ representa o valor esperado dos bins dos modelos de MUED.

Note que, agora, colocamos o modelo de supersimetria na hipótese nula, isso quer dizer que estamos beneficiando supersimetria, pois o erro de falsa aceitação de supersimetria é fixo (tipo-I). Em bases menos exigentes, teríamos de assumir que MUED poderia estar também na hipótese nula.

Assim 2.35 torna-se

$$
\Lambda=\sum_{i=1}^{n_{\text {dist }}} \sum_{j=1}^{n_{\text {bins }}} 2\left(s_{i j}-u_{i j}-r_{i j} \ln \frac{s_{i j}+b_{i j}}{u_{i j}+b_{i j}}\right) .
$$

\subsection{Métricas de significância, $Z_{s b}$ e $Z_{L L R}$}

A contagem de eventos é a forma mais direta de buscar um sinal de nova física, porém essa contagem, assim como qualquer outro número obtido de um dado experimental, está sujeita a flutuações do tipo Poisson. Assumindo que um dado experimento tenha um background e sinal total, esperados, de $b$ e $s$ eventos, respectivamente, uma métrica de significância usual para o sinal observado $s$ é $Z_{s b}$ :

$$
Z_{s b}=\frac{s}{\sqrt{b}}
$$


Esta fórmula é obtida facilmente no caso em que as PDFs dos números de eventos (Poisson) podem ser aproximadas por distribuições Gaussianas. Suponha que $s+b=b+Z_{s b} \sigma_{b}$, a probabilidade $\alpha$ de que $b$ possa flutuar para valores, pelo menos iguais a $b+N_{\sigma} b$, é a integral:

$$
\alpha=\int_{b+Z_{s b} \sqrt{b}}^{+\infty} \mathcal{G}(b, \sqrt{b} \mid x) d x
$$

justamente o nível de significância do teste, e de onde vem que $Z_{s b}=s / \sigma_{b}=s / \sqrt{b}$. A função $\mathcal{G}(b, \sqrt{b} \mid x)$ é uma gaussiana normalizada a unidade, de média $b$ e desvio padrão $\sqrt{b}$.

Apesar de sua simplicidade, esta métrica tem a desvantagem de superestimar a significância estatística se o número de eventos observados não é muito grande. Além disso, a não ser que $b>s, Z_{s b}$ também superestima a significância estatística em relação ao cálculo exato com uma Poisson, uma vez que a cauda da Poisson é mais pesada do que a da Gaussiana, ou seja, estende-se por um valor de abcissa maior. Veja a Fig. (2.9).

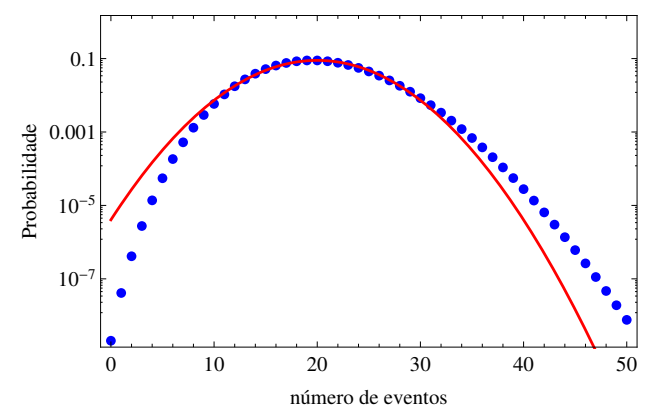

Figura 2.9: Curvas para a distribuição Gaussiana (vermelha-contínua) e distribuição de Poisson (ponto-azul) em escala logarítmica.

Em nosso caso, o número de eventos depende da luminosidade integrada, correções NLO, eficiência de cortes e tagging de jatos de quarks e glúons. Todos esses fatores podem influenciar no valor de $Z_{s b}$.

Quando, além da incerteza estatística, espera-se uma incerteza sistemática na taxa dos eventos, digamos de $\epsilon$, podemos adicionar à incerteza estatística $\sqrt{b}$ uma incerteza sistemática na taxa dos eventos de background $\epsilon b$, na forma de soma em quadratura [15], de modo que a incerteza total será

$$
\sigma_{T}=\sqrt{(\sqrt{b})^{2}+(\epsilon b)^{2}} .
$$

Assim 2.40 modifica-se para,

$$
Z_{s b}^{\text {sist }}=\frac{s}{\sqrt{\left(b+(\epsilon b)^{2}\right.}} .
$$

Para efeito de comparação, vamos calcular também a significância de um sinal $s$ em relação a um background $b$ usando a estatística de Poisson pela fórmula11

$$
\begin{aligned}
\alpha & =\sum_{k=s+b}^{\infty} \mathcal{P}(k \mid b), \\
Z_{P} & =\Phi(1-\alpha) .
\end{aligned}
$$

Marginalizando sobre a incerteza sistemática através de uma distribuição Gaussiana de acordo com:

$$
\begin{aligned}
\alpha & =\sum_{k=s+b}^{\infty} \int_{-5}^{5} \mathcal{P}(k \mid b *(1+\theta \epsilon)) \times \frac{e^{-\theta^{2} / 2}}{\sqrt{2 \pi}} d \theta \\
Z_{P}^{\text {sist }} & =\Phi(1-\alpha)
\end{aligned}
$$

$$
{ }^{1} \Phi(x)=\frac{1}{2 \sqrt{\pi}} \int_{-\infty}^{x} e^{\frac{-x^{2}}{2}} .
$$


Analisamos o comportamento de $Z_{s b}, Z_{s b}^{\text {sist }}, Z_{P}$ e $Z_{P}^{\text {sist }}$ com um erro sistemático $\epsilon$ na taxa dos eventos de background. Na Fig. 2.10 temos uma comparação de $(2.40-2.45$, para seções de choque típicas encontradas nesse trabalho $\left(\sigma_{\text {susy }}=0.34 \mathrm{fb}\right.$ e $\left.\sigma_{b c k g}=0.88 \mathrm{fb}\right)$ e luminosidade de $100 \mathrm{fb}^{-1}$, em função da incerteza sistemática.

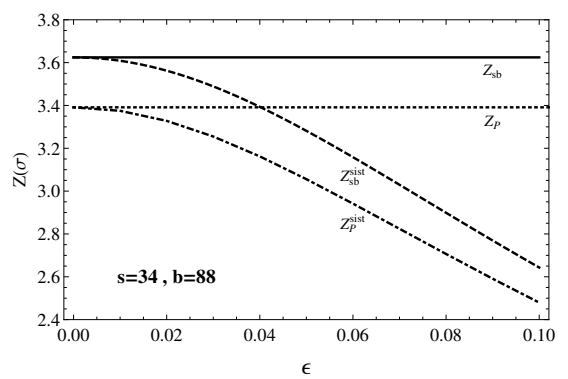

Figura 2.10: Comparação entre o teste $Z_{s b}$ no caso com e sem erro sistemático na taxa dos eventos de background.

Em primeiro lugar, confirmamos que $Z_{s b}$ superestima a significância estatística em qualquer caso. Para uma incerteza sistemática de 10\%, por exemplo, há uma diferença de aproximadamente $1 \sigma$ entre $Z_{s b}$ e $Z_{s b}^{\text {sist }}$, as linhas sólida e tracejada, respectivamente, e que cresce com a incerteza. Em comparação ao cálculo exato com a estatística de Poisson, sem incertezas sistemáticas, a diferença entre $Z_{s b}$ e $Z_{P}$ (a linha pontilhada) é de cerca de $0.2 \sigma$. Nesse caso, as diferenças aumentam se $s$ e $b$ são próximos e pequenos $(<100)$.

Note, finalmente, que a diferença entre as métricas baseadas nas distribuições de Poisson e Gaussiana, na presença de incertezas sistemáticas, $Z_{s b}^{\text {sist }}$ e $Z_{P}^{\text {sist }}$ (linha ponto-traço), respectivamente, é menor do que a correspondente diferença sem incertezas sistemáticas. Isso mostra a importância de se levar em conta tais incertezas no cálculo da significância estatística.

Para contornar as limitações impostas pelos erros sistemáticos, é necessário aumentar a razão sinal sobre background. Podemos manipular a equação 2.42 e determinarmos uma relação de vínculo entre $\frac{s}{b}$, a fração do número de eventos de sinal com relação ao background, e a incerteza sistemática $\epsilon$,

$$
\frac{s}{b}=\frac{2 \epsilon^{2}}{-\frac{1}{s}+\sqrt{\left(\frac{1}{s}\right)^{2}+4 \frac{\epsilon^{2}}{\left(Z_{s b}^{s i s}\right)^{2}}}}
$$

Vemos que a incerteza sistemática na taxa dos eventos coloca uma limitação no poder de descoberta de nova física. Veja a Fig. 2.11, nela temos três casos para 2.46 , com $Z_{s b}^{\text {sist }}=1$, 3 e 5, onde escolhemos uma seção de choque para o sinal de $1 \mathrm{fb}$, e luminosidade integrada de $100 \mathrm{fb}^{-1}$. Observe que, a razão $\frac{s}{b}$ necessária para alcançar determinada significância depende criticamente do nível de erros sistemáticos do experimento. Em um cenário de descoberta de nova física, por exemplo, com uma incerteza sistemática de $10 \%$, teremos significância igual a $5 \sigma$ somente se a razão entre sinal e background for maior que 90\%. Se, eventualmente, a separação de sinal e background não for tão efetiva, então nunca poderemos alcançar descoberta de nova física nesse canal usando a simples contagem de eventos.

\subsubsection{A significância calculada com base na estatística de verossimilhança}

Retomando o log-likelihood ratio para descoberta e discernimento 2.37 e 2.39, vemos que para definir a estatística-teste é necessário determinar $r_{j i}$. Conforme definimos anteriormente, $s_{i j}$ e $b_{i j}$ correspondem às nossas predições teóricas oriundas das simulações de Monte Carlo. Esses valores ficam fixos após uma simulação.

Ao simularmos um grande número de pseudo-experimentos, obtemos uma coleção de valores da estatística-teste, no caso, o log-likelihood ratio. Organizando estes valores em bins de 


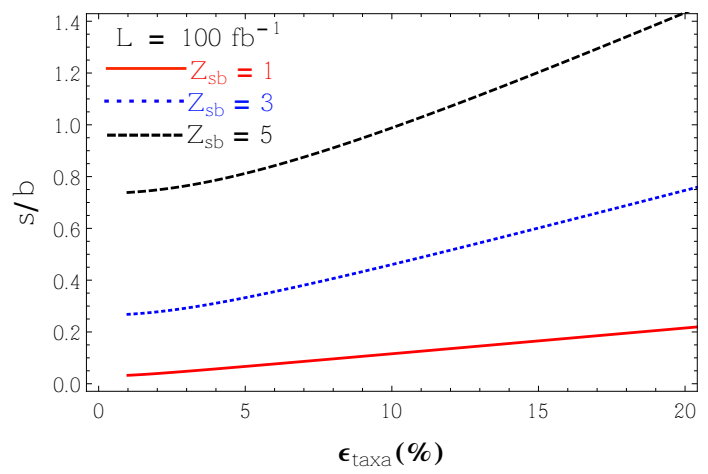

Figura 2.11: Fração de sinal e background necessária para alcançar uma dada $Z_{s b}$, para incerteza sistemática na taxa dos eventos de background $\epsilon_{\text {taxa }}$ e luminosidade de $100 \mathrm{fb}^{-1}$.

um histograma podemos ajustar uma função às entradas deste histograma. Tal função será a nossa estimativa da PDF da estatística: $f(\Lambda)$ para um determinado modelo - seja backgrounds $\left(\mathrm{f}\left(\Lambda \mid H_{0}\right)\right)$ ou sinal+backgrounds $\left(\mathrm{f}\left(\Lambda \mid H_{1}\right)\right)$.

Como os valores de $r_{i j}$ são a manifestação experimental de um dado modelo, neles devem ser inseridos, além da flutuação estatística inerente de todo dado experimental, as incertezas sistemáticas.

Um resultado importante para a análise dos pseudo-experimentos vem do teorema Central do Limite. A distribuição de uma soma de $n$ variáveis aleatórias i.i.d. (identicamente, independentemente distribuídas), cada uma com média $\mu_{i}$ e variância $\sigma_{i}{ }^{2}$, no limite de $n$ infinito, tende a uma distribuição gaussiana com média $\mu=\sum_{i=1}^{n} \mu_{i}$ e variância $\sigma^{2}=\sum_{i=1}^{n} \sigma_{i}{ }^{2}$. Qualquer soma de variáveis aleatórias que estejam definidas por qualquer PDF, em um dado limite de muitos dados, tendem sempre a uma distribuição gaussiana. Pelas equações $2.37 \mathrm{e} 2.39$, vemos que a estatística tem, basicamente, a estrutura $\Lambda=C+\sum_{i} \sum_{j} r_{i j} w_{i j}, C$ uma constante, $r_{i j} \sim \mathcal{P}\left(\mu_{i j}\right)$ e $w_{i j}$ pesos fixos que dependem apenas dos $s_{i j}, u_{i j}, b_{i j}$. Observamos que com 300.000 pseudo-experimentos, a convergência das PDFs da estatística-teste $\Lambda$ para uma gaussiana é muito boa.

É útil identificar a área sob uma PDF em termos do número de desvios padrões de uma Normal padrão $(Z)$. Considere a distribuição normal de média 0 e desvio padrão $1, f(x)=\frac{1}{\sqrt{2 \pi}} e^{-x^{2} / 2}$, a função definida por

$$
\Phi(x)=\int_{-\infty}^{x} f(t) d t
$$

é a distribuição cumulativa da PDF Normal padrão (Cumulative Distribution Function, CDF em inglês).

Tendo calculado a área sob uma PDF, digamos $\alpha$, basta agora resolver a equação

$$
1-\Phi(Z)=\alpha
$$

de modo a obter $Z=\Phi^{-1}(1-\alpha)$. A significância $\alpha$ fica assim associada ao número equivalente de desvios padrões da média da Normal padrão, $Z$.

Na Fig. 2.12 temos a PDF para hipótese nula $\left(H_{0}\right)$, gerada em uma análise de descoberta de supersimetria. Verificamos que o comportamento gaussiano da PDF do log-likelihood ratio se estende consideravelmente em sua cauda. Simulando $10^{7}$ pseudo-experimentos, mostramos a comparação entre $f(\Lambda)$ obtida numericamente e uma gaussiana com média e desvio padrão que melhor se ajusta a esta PDF na Fig. 2.13. Vemos que a gaussiana criada a partir do histograma tem, para um dado $\Lambda_{O B S}$, maior valor de erro do tipo-I $(\alpha)$. Isso garante que a significância medida pela gaussiana teórica é menor do que a obtida através do histograma, novamente estamos sendo conservadores na análise. Tendo confiança de que $\Lambda$ segue uma distribuição Normal, 


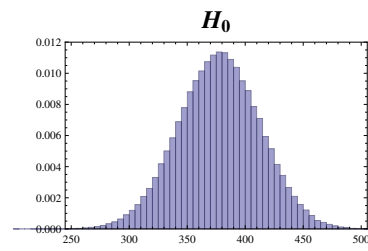

$\Lambda$

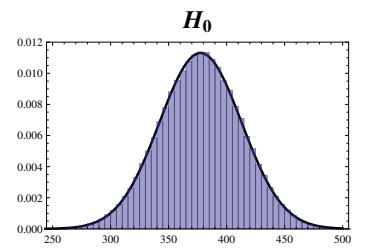

$\Lambda$

Figura 2.12: PDF para hipótese nula (backgrounds). À esquerda somente o histograma, à direita o histograma fitado por uma gaussiana de média e desvio padrão apropriados.

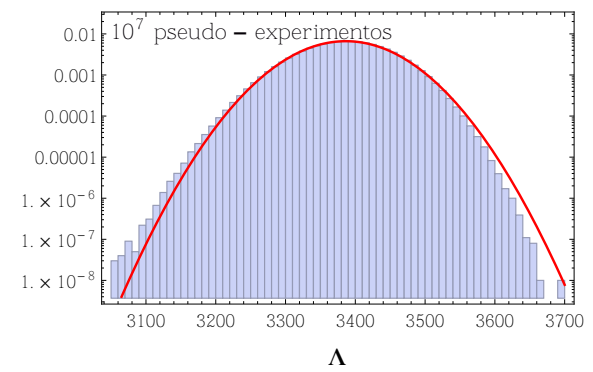

Figura 2.13: Simulação para $10^{7}$ pseudo-experimentos. Na escala logarítmica esboçamos a curva gaussiana e seu histograma associado. Vemos que na região da cauda direita a área dada pela gaussiana tende a ser maior que a contagem de bins do histograma. Isso garante que a significância, para uma dada luminosidade, é menor para a gaussiana.

simulamos para cada espectro estudado 300.000 pseudo-experimentos de modo a estimar a média e a variância de $f(\Lambda)$ e assim calcular a significância de forma mais simples e rápida.

O cálculo da significância da estatística-teste log-likelihood ratio é simplificado quando as PDFs são distribuições gaussianas, e o valor observado da estatística-teste situa-se na média da hipótese alternativa [16]. Na Fig. 2.14 temos esboçadas a hipótese nula (backgrounds) e alternativa (supersimetria + backgrounds) para uma análise de descoberta de supersimetria. $Z_{L L R}$, nesse caso, onde as PDFs das hipóteses são gaussianas é calculada facilmente por

$$
Z_{\mathrm{LLR}}=\frac{\left|<\Lambda_{H_{0}}>-<\Lambda_{H_{1}}>\right|}{\sigma_{\Lambda_{H_{0}}}} .
$$

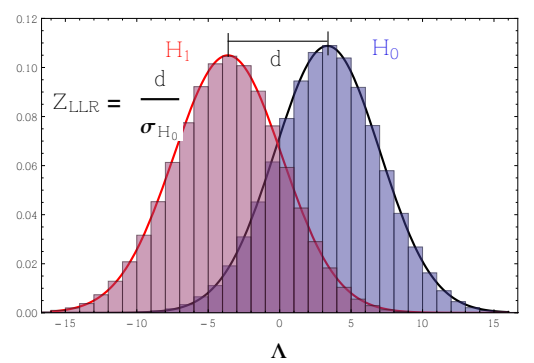

Figura 2.14: A métrica de significância para o teste de hipóteses. Note que as PDF's possuem limites gaussianos e a equação para $Z_{L L R}$ torna-se a simples distância entre as hipóteses em unidades de $\sigma_{H_{0}}$.

Além da distância entre as médias das distribuições de $\Lambda$ sob a hipótese de $H_{0}$ e de $H_{1}$, a significância aumenta de forma inversamente proporcional ao desvio padrão de $f\left(\Lambda \mid H_{0}\right)$. Quando há incertezas sistemáticas nas normalizações, como às que estudamos, a diferença $\mid\left\langle\Lambda_{H_{0}}\right\rangle$ $-<\Lambda_{H_{1}}>\mid$ permanece inalterada em relação ao cálculo sem as incertezas, porém, $\sigma_{H_{0}}$ aumenta 


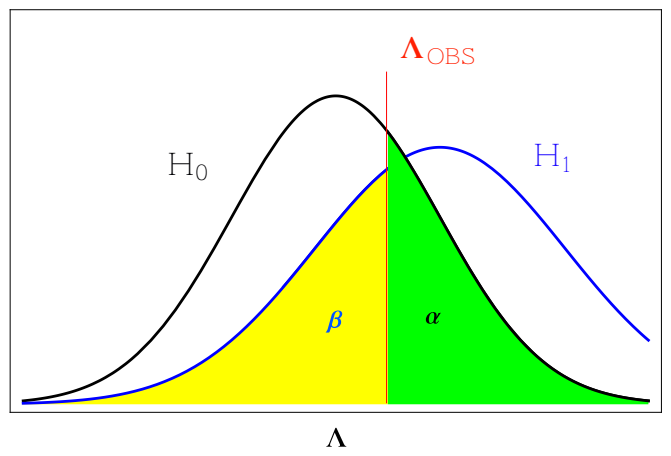

Figura 2.15: Cálculo dos erros do tipo-I e tipo-II, $\Lambda_{\mathrm{CUT}}$ é definido arbitrariamente.

tornando $f\left(\Lambda_{H_{0}}\right)$ mais larga e, por conseguinte, diminuindo a significância do teste. Vamos tratar do efeito das incertezas sistemáticas na próxima seção.

Na Fig. 2.15 vemos como o cálculo dos erros do tipo I e II é feito, usando as PDFs fitadas para cada uma das hipóteses, no caso de um $\Lambda_{O B S}$ qualquer. É comum assumir a convenção de usar o $\Lambda_{O B S}$ como sendo a média da hipótese alternativa. Isso garante um erro do tipo-II de, sempre, $50 \%$.

\subsection{Marginalização dos erros sistemáticos}

O teorema de Bayes afirma que:

$$
p(H \mid \text { resultado })=\frac{L(\text { resultado } \mid H) \pi(H)}{p(\text { resultado })} .
$$

Onde,

$$
p(\text { resultado })=\sum_{i} p\left(\text { resultado } \mid H_{i}\right) p\left(H_{i}\right)
$$

Aqui $L($ resultado $\mid H)$ é o likelihood para observação de um dado resultado sob a hipótese de um modelo $H . \quad \pi(H)$ é o prior para o modelo $H$, quantifica o quanto acreditamos ou inferimos sobre esse modelo antes de qualquer evidência ou informação sobre o modelo. E finalmente $p(H \mid$ resultado $)$ é o posterior, a probabilidade atualizada do modelo $H$ ser o responsável pela medida de resultado após levarmos em conta qualquer informação sobre ele.

Consideram-se incertezas sistemáticas quaisquer incertezas que não tenham origem estatística. Em geral, uma série de experimentos repetidos diminuem uma incerteza do tipo estatística, porém incertezas sistemáticas não seguem essa regra. Essas incertezas podem afetar somente o sinal, somente o backgroud ou ambos ao mesmo tempo. Isso dependerá do tipo de incerteza em questão. Por exemplo, a incerteza na luminosidade integrada influencia o número de eventos de todas as amostras de sinal e background do mesmo modo, essa incerteza é dita correlacionada entre sinal e background.

A inserção de incertezas sistemáticas no likelihood pode ser feita através de marginalização do likelihood ou profiling likelihood. O segundo é mais usado em casos de determinação de parâmetros desconhecidos, e por isso é usado em hipóteses compostas. A marginalização oferece uma forma mais rápida de inserir incertezas em nosso caso, pois baseia-se no teorema de Bayes. O posterior, dado pela equação de Bayes (2.49), pode denotar o efeito de uma incerteza sistemática desde que integremos o likelihood apropriadamente. Em nosso caso assumiremos que as incertezas sistemáticas apresentam-se nos likelihoods na forma do que se chama (nuisance parameters) parâmetros que não são alvos diretos da inferência, mas que devem ser incorporados na análise relativa aos parâmetros de interesse. 
Aplicamos incertezas sistemáticas na normalização (taxa) e na forma das distribuições [66]. Dentre as incertezas que afetam as taxa de nossos eventos consideramos incertezas: na luminosidade integrada, afeta sinal e background de forma simétrica; nas PDFs parton distribution function $2^{2}$, afeta sinal e background de forma assimétrica. Já na forma das distribuições levamos em conta uma incerteza devido à baixa eficiência de Monte Carlo após cortes retangulares duros para o background. Retomaremos essa discussão, apropriadamente, no próximo capítulo, usando os dados que utilizamos para obter nossos resultados.

Assumiremos que as incertezas nas normalizações são distribuídas de forma normal. As incertezas na forma das distribuições serão aplicadas através de funções de Poisson cujos parâmetros serão dados pela previsão teórica de nossas simulações em cada bin. Ressaltamos que incertezas sistemáticas na taxa dos eventos podem alterar também as formas das distribuições, veja [66], esse efeito foi desconsiderado em nossa aplicação de incertezas nas taxas dos eventos.

O posterior 2.49), para o likelihood 2.34), com priors apropriados para cada uma das incertezas sistemáticas, pode ser considerado como o likelihood marginalizado [69], e esse likelihood pode ser usado para inferir $Z_{L L R}$, o $p$-value e o poder de nosso teste, assim como os usados em uma análise frequentista. Esse processo de inserção de incertezas sistemáticas, através de marginalização do likelihood, é conhecido como híbrido Bayesiano-frequentista. O likelihood marginalizado com incertezas na taxa dos eventos será,

$$
L_{m} \equiv \int L(N \mid s(\vec{\theta}), b(\vec{\theta})) \eta(\vec{\theta}) d \vec{\theta} .
$$

$\vec{\theta}$ é um conjunto de incertezas sistemáticas na taxa e as funções $\eta(\vec{\theta})$ são os priors gaussianos, com média 0 e desvio padrão 1. Para a marginalização de incertezas no formato das distribuições, basta alterar a função $\eta(\vec{\theta})$, o prior, para uma distribuição de Poisson [71].

Uma grande vantagem do likelihood é que a aplicação de diversas incertezas sistemáticas pode ser feita de forma conjunta, sempre de maneira multiplicativa. No próximo capítulo, iremos contextualizar os tipos de incertezas necessárias para uma boa descrição de nosso trabalho, assim como a aplicação das incertezas sistemáticas no likelihood.

\footnotetext{
${ }^{2}$ As incertezas nas PDFs também podem afetar a forma das distribuições cinemáticas, estamos considerando o efeito dessas incertezas somente na normalização das distribuições [66].
} 


\section{Capítulo 3}

\section{Análise}

Em colisões próton-próton, como as que ocorrem no LHC, partículas carregadas sob o grupo $S U(3)_{C}$ da QCD são produzidas copiosamente. Em especial, mesmo novas partículas pesadas, como squarks e gluinos, que interagem fortemente com quarks e glúons, devem ter seções de choque de produção grande comparadas com outras partículas produzidas de forma eletro-fraca, como sléptons e gauginos, por exemplo. Isso, é claro, torna a procura por partículas coloridas, um alvo para as colaborações experimentais. Contudo, na maioria dos modelos de nova física, tais partículas são instáveis e decaem rapidamente levando invariavelmente à produção de jatos. No caso de squarks, gluinos ou quarks e glúons excitados de modelos de dimensões extras universais, além dos jatos haverá a produção de duas LSP (LKP), a candidata à matéria escura desses modelos. Isso nos traz ao estudo de um canal clássico de procura, jatos + MET.

Neste capítulo iremos descrever nossas simulações e os aspectos estatísticos relevantes ao nosso trabalho. Descreveremos a escolha do tipo de sinal para procura de nova física e seus canais de produção para o LHC. Passaremos pela simulação e seleção de eventos, assim como a descrição das variáveis usadas para construção dos observáveis físicos. Posteriormente descreveremos a análise estatística assim como a inserção de incertezas sistemáticas.

Como guia geral, o fluxograma da Fig. 3.1 ilustra de forma esquemática todo o transcorrer da análise junto com os programas e rotinas usadas em cada passo. Iniciamos o análise criando os espectros de nova física com o programa SPHENo 42, logo em seguida iniciamos a simulação dos eventos e integração no espaço de fases com o programa MADGRAPH5 [81] para o LHC 14 $\mathrm{TeV}$, usando modelos de supersimetria e MUED criados a partir do programa FeynRules [67]. Após a produção dos eventos a nível partônico, fizemos o parton shower e hadronização com o programa PүтніА 82 versão 6, subsequentemente simulamos efeitos de detecção com o programa PGS [83]. Com os eventos devidamente simulados começamos o escaneamento de cortes retangulares, usando distribuições cinemáticas criadas no programa MadAnalysis [68]. Definido os cortes, passamos para a aplicação de eficiências de detecção, correções NLO (Prospino) [94], a aplicação do tagging de jatos de quarks e glúons [21, 22], e normalização das distribuições de MUED. A partir daqui começamos a gerar os pseudo-experimentos necessários para definição da estatística-teste do teste de hipóteses, juntamente com a inserção de erros sistemáticos na taxa e forma dos observáveis físicos. Finalmente usamos esse conjunto de informações para definir a significância do teste de hipóteses e analisar a descoberta e discernimento de supersimetria e MUED.

A simulação e análise de resultados envolveu uma quantidade muito grande de processamento computacional, programação, automação e concatenação de diversas ferramentas. Isso demandou a geração de milhões de eventos partônicos além de seu pós-processamento visando a simulação de efeitos de hadronização de quarks e glúons, identificação de jatos, e efeitos de detectores. Finalmente, na parte final da análise, um segundo e demorado esforço computacional foi imprescindível para a estimativa do impacto de alguns erros sistemáticos importantes no cálculo da métrica de significância esperada tanto na descoberta da nova física quanto no nosso principal 
objetivo, o discernimento entre os modelos supersimétrico e de dimensões extras universais.

Para melhor ilustração de algumas discussões que iremos realizar no início desse capítulo, faz-se necessário a definição de 3 espectros fixos de supersimetria e MUED,

- Espectro A: $m_{\tilde{q}}=m_{q^{(1)}}=1.4 \mathrm{TeV}, m_{\tilde{g}}=m_{g^{(1)}}=1.5 \mathrm{TeV}$ e $m_{\tilde{\chi}_{1}}=m_{B^{(1)}}=0.3 \mathrm{TeV}$.

- Espectro B: $m_{\tilde{q}}=m_{q^{(1)}}=2.6 \mathrm{TeV}, m_{\tilde{g}}=m_{g^{(1)}}=4.0 \mathrm{TeV}$ e $m_{\tilde{\chi}_{1}}=m_{B^{(1)}}=0.3 \mathrm{TeV}$.

- Espectro C: $m_{\tilde{q}}=m_{q^{(1)}}=1.4 \mathrm{TeV}, m_{\tilde{g}}=m_{g^{(1)}}=1.5 \mathrm{TeV}$ e $m_{\tilde{\chi}_{1}}=m_{B^{(1)}}=1 \mathrm{TeV}$.

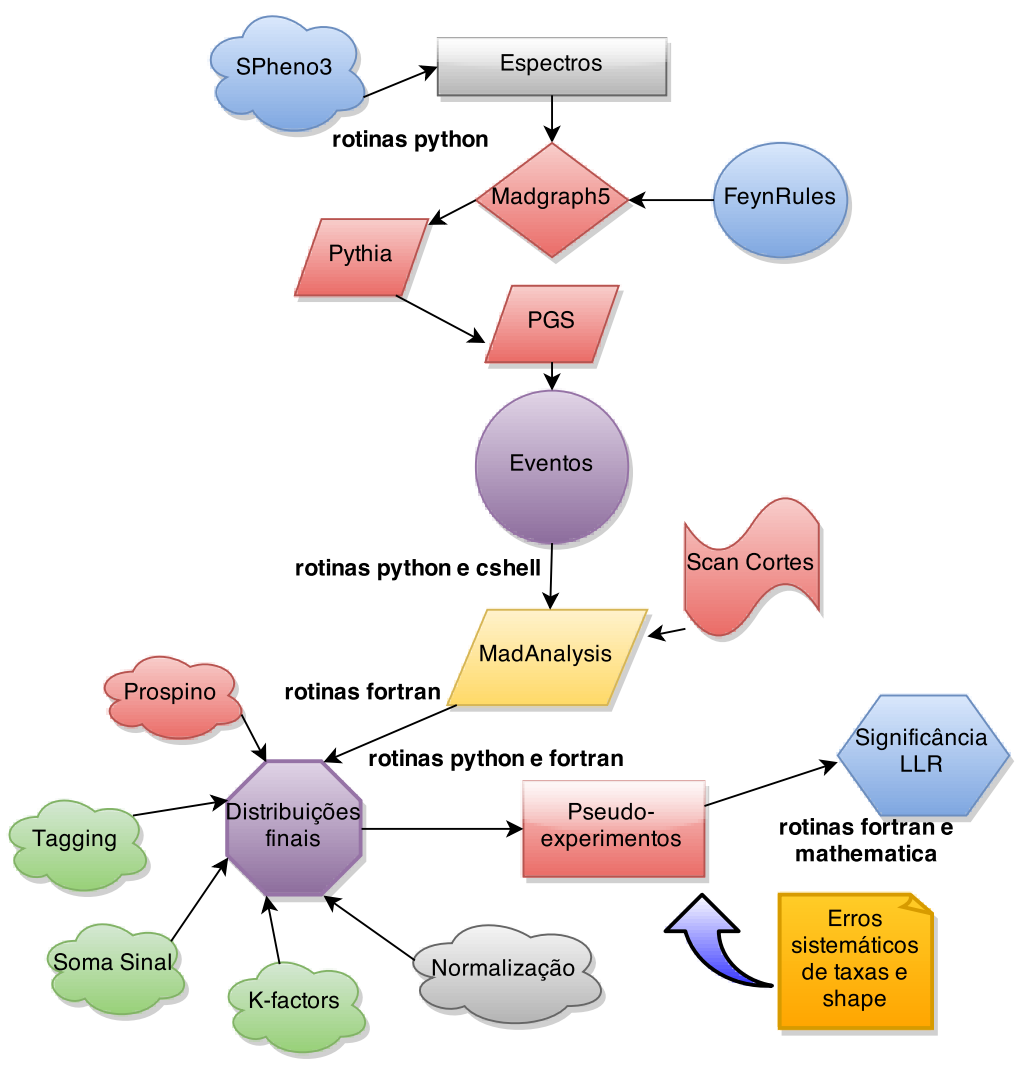

Figura 3.1: Fluxograma esquemático de todo o trabalho realizado.

\subsection{Produção de squarks no LHC}

As produções de squarks e gluinos no LHC ocorrem através de uma série de processos envolvendo quarks e glúons no estado inicial, veja as figuras 3.2 e 3.3 . Na Fig. 3.4 mostramos as seções de choque de produção de $\tilde{q}_{i}^{\prime} \tilde{q}_{j}^{\prime}, \tilde{q}_{i}^{\prime} \tilde{g}, i, j=L, R$, e $\tilde{g} \tilde{g}$ no LHC $14 \mathrm{TeV}$. De uma forma geral, as seções de choque caem rapidamente com o aumento da massa dos squarks e gluinos. Na tabela (3.1) mostramos alguns dos espectros que analisamos nesse trabalho junto com a contribuição relativa da seção de choque de cada canal, comparando entre alguns dos pontos mais leves e mais pesados. Na região onde $m_{\tilde{g}}<m_{\tilde{q}}$ temos um favorecimento à produção de gluinos, a produção associada de squarks e gluinos é um processo sub-dominante nesse cenário e a produção de squarks é pequena. Já na região $m_{\tilde{g}}>m_{\tilde{q}}$ a produção de squarks domina, a produção associada de squarks e gluinos torna-se sub-dominante e a produção de gluinos é pequena. 


\begin{tabular}{cccccc}
\hline$m_{\tilde{q}}(\mathrm{TeV})$ & $m_{\tilde{g}}(\mathrm{TeV})$ & $\frac{\sigma_{\tilde{q} \tilde{q}}}{\sigma}(\%)$ & $\frac{\sigma_{\tilde{q} \tilde{q}^{*}}}{\sigma}(\%)$ & $\frac{\sigma_{\tilde{q} \tilde{g}}}{\sigma}(\%)$ & $\frac{\sigma_{\tilde{g} \tilde{g}}}{\sigma}(\%)$ \\
\hline \hline 1.4 & 1.5 & 38 & 18 & 44 & 0 \\
1.4 & 2.0 & 57 & 20 & 22 & 0 \\
1.4 & 3.0 & 70 & 29 & 1 & 0 \\
1.4 & 4.0 & 66 & 33 & 1 & 0 \\
1.4 & 5.0 & 62 & 38 & 0 & 0 \\
5.0 & 1.5 & 0 & 0 & 0 & 100 \\
5.0 & 2.0 & 0 & 0 & 1 & 99 \\
5.0 & 3.0 & 0 & 0 & 12 & 88 \\
5.0 & 4.0 & 7 & 0 & 54 & 39 \\
5.0 & 5.1 & 78 & 2 & 19 & 0 \\
\hline \hline
\end{tabular}

Tabela 3.1: Contribuições relativas para as seções de choque em NLO de supersimetria, onde $\sigma=\sigma_{\tilde{q} \tilde{q}}+\sigma_{\tilde{q} \tilde{q}^{*}}+\sigma_{\tilde{q} \tilde{g}}+\sigma_{\tilde{g} \tilde{g}}$

O CMS e o ATLAS têm realizado análises de procura de supersimetria em modelos supersimétricos simplificados para produção de partículas coloridas no regime de desacoplamento dos gluinos [91, 92, 93]. Nesse regime, os squarks são produzidos predominantemente pela interação direta com glúons, veja a Fig. 3.2, em especial os diagramas sensíveis ao desacoplamento de gluinos, os $g g \rightarrow \tilde{q} \tilde{q}$. Ainda na Fig. 3.2 temos uma contribuição vinda diretamente dos quarks
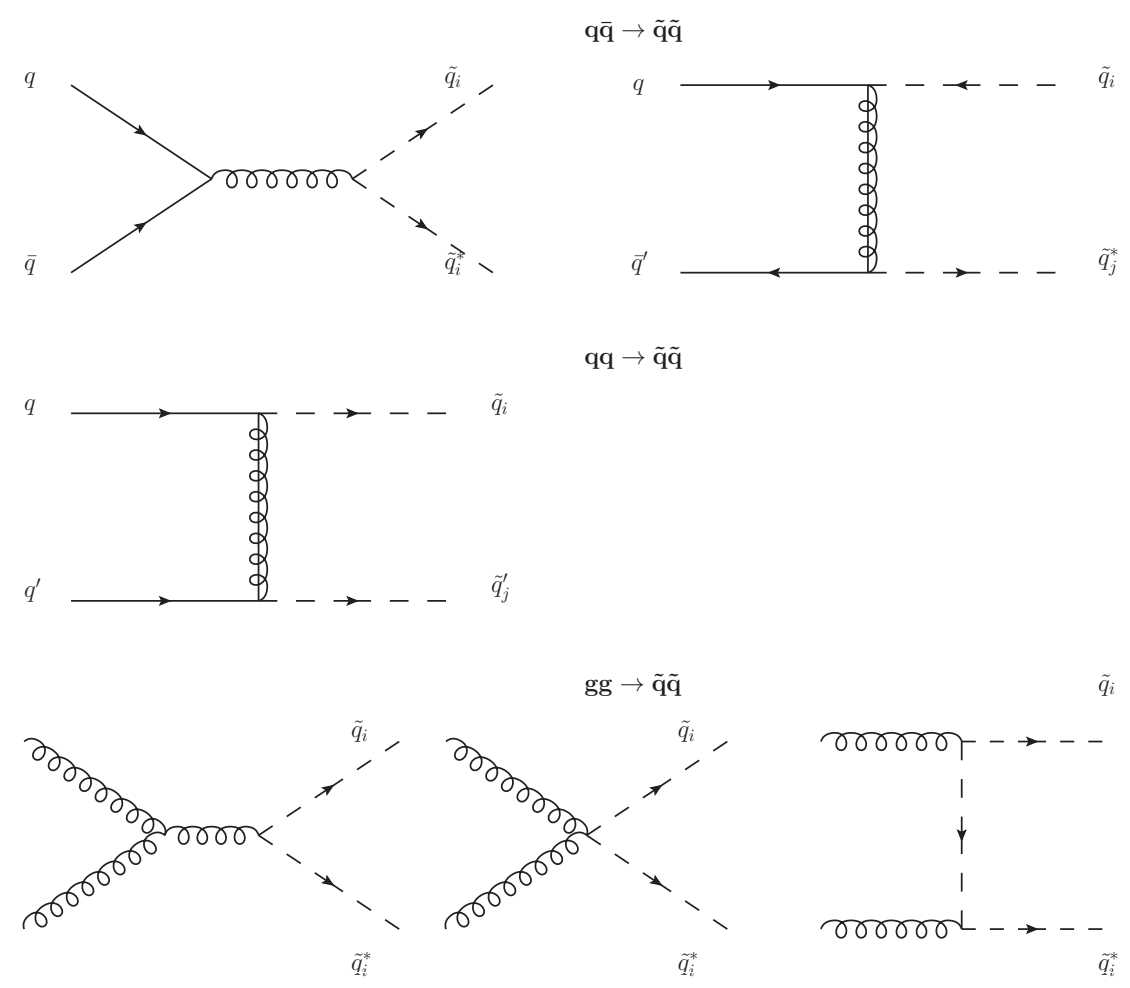

Figura 3.2: Diagramas de Feynman para produção de squarks no LHC. O único diagrama que contribui no canal $q q \rightarrow \tilde{q} \tilde{q}$ é o canal dominante na produção de squarks.

de valência, a $q q^{\prime} \rightarrow \tilde{q} \tilde{q}$ com um gluino no canal-t. Podemos esperar que, enquanto tenha-se gluinos não muito mais pesados que squarks, esses diagramas serão predominantes na produção de squarks para o LHC, além de que, a grande multiplicidade de possíveis estados iniciais envolvendo sabores de quarks distintos, para esse processo, leva também a um aumento da seção de choque final. 
Já na Fig. 3.3, temos os diagramas de Feynman responsáveis pela produção de squarks e gluinos. Vemos que estados iniciais somente com quarks ou glúons não contribuem nesse processo.

Recentemente mostrou-se [90] que o limite de desacoplamento de gluinos possui apenas um acordo moderado com o regime não desacoplado. Uma análise onde o gluino não esteja desacoplado mostra-se mais adequada no regime de massas que estamos analisando, uma vez que estamos interessados em regimes de partículas de ordem de 1 a $5 \mathrm{TeV}$, com hierarquias fortes e fracas entre os squarks, os gluinos e o neutralino mais leve.

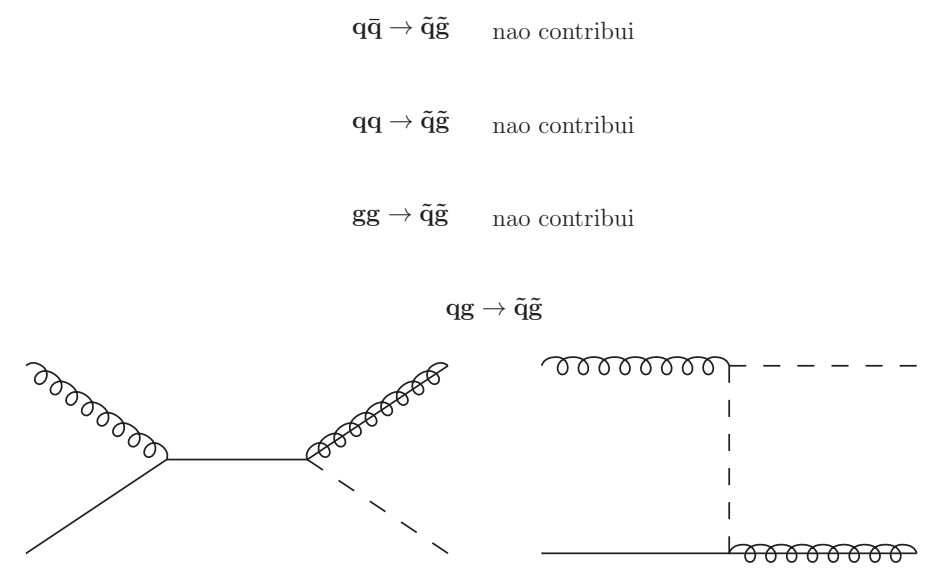

Figura 3.3: Diagramas de Feynman para produção de squarks e gluinos no LHC.
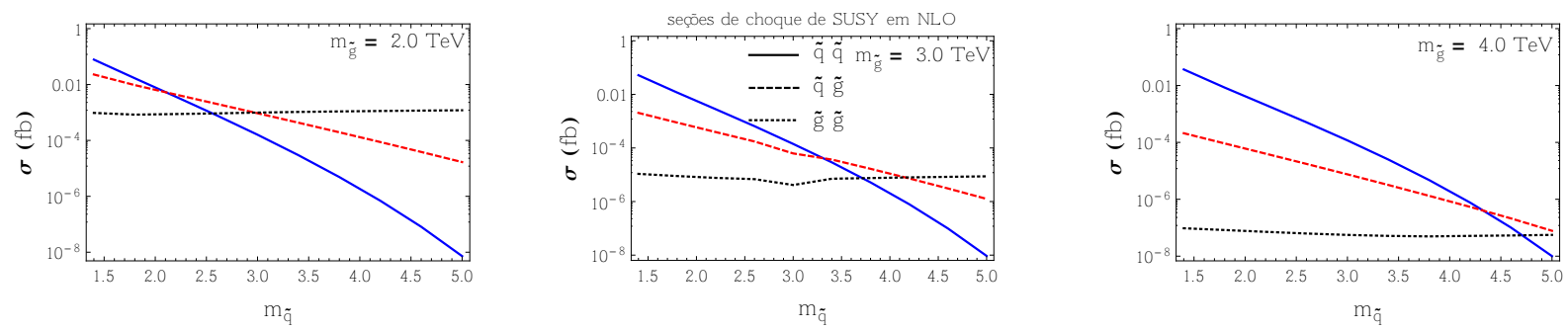

Figura 3.4: Seções de choque em NLO para produção de squarks e gluinos em função da massas dos squarks e gluinos. Valores calculados através do programa Prospino.

Para verificarmos se, de fato, a contribuição de gluinos no canal-t é dominante em nossos observáveis físicos, fizemos uma avaliação da contribuição relativa de gluinos, veja Fig. 3.5, através do cálculo dos processos listados na Fig 3.2 para a produção de squarks no LHC. O primeiro processo é a contribuição advinda somente de glúons no estado inicial, definimos como a curva sem quarks,

$$
g g \underset{\text { intermediárias }}{\stackrel{\text { todas partículas }}{\longrightarrow}} \tilde{q} \tilde{q}^{\prime} .
$$

O segundo com processos iniciados apenas por quarks com contribuição somente do canal-s, esse processo excluí gluinos no canal-t de produção de squarks (denominado curva sem glúons, sem $\tilde{g})$ :

$$
q \bar{q} \underset{\text { somente canal-s }}{\stackrel{\text { sem g e sem } \tilde{q}}{\longrightarrow}} \tilde{q} \tilde{q}^{\prime} .
$$

O terceiro é o processo iniciado somente por quarks e apenas canal-t com gluinos (curva sem glúons + canal-t gluinos):

$$
q q \underset{\text { somente canal-t }}{\stackrel{\operatorname{com} \tilde{g}}{\longrightarrow}} \tilde{q} \tilde{q}^{\prime} .
$$

Note que na Fig. (3.5) as curvas sem glúons no estado inicial e somente com gluinos no canal-t são idênticas à curva da contribuição total todos os canais. Se as distribuições não estivessem 

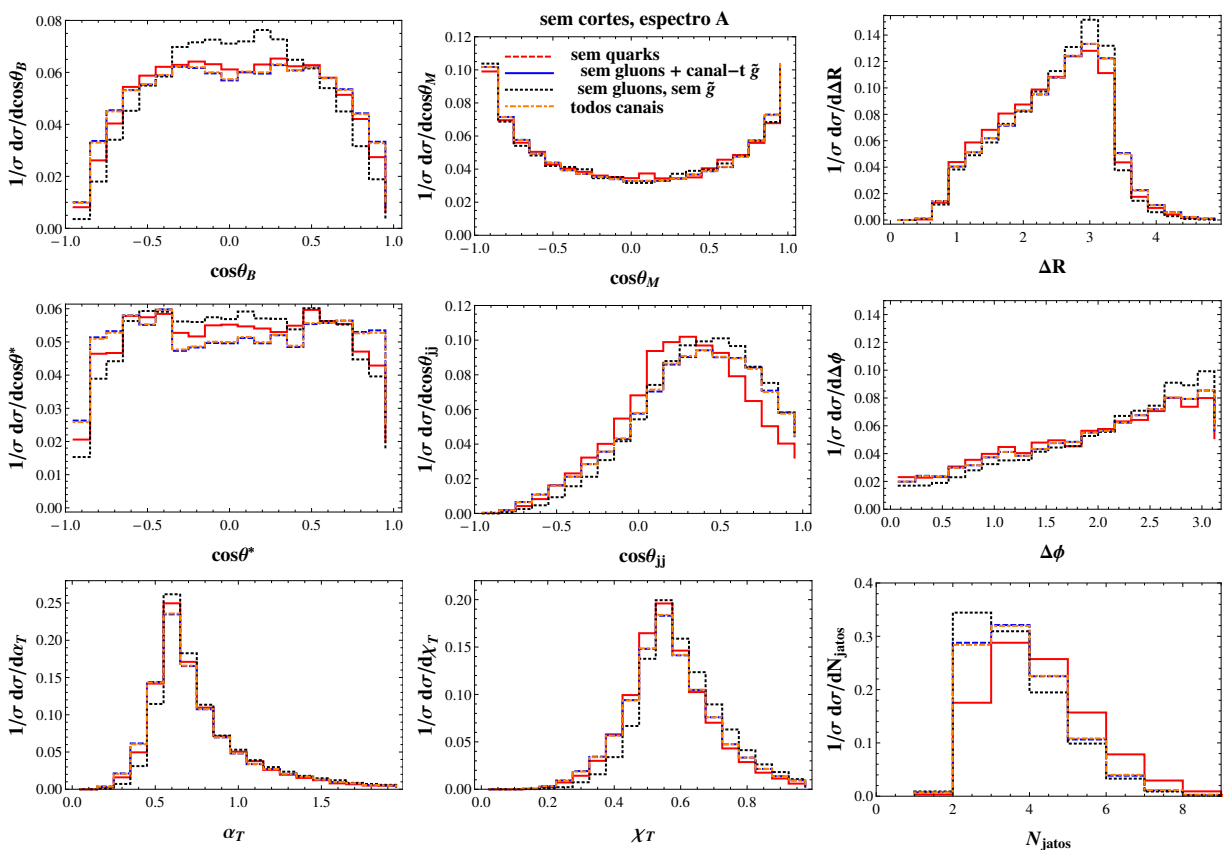

Figura 3.5: Distribuições normalizadas para produção de squarks no LHC. Contribuições do canal-t vindas dos gluinos são as que dominam as seções de choque para produção de squarks no LHC. As distribuições cinemáticas mostradas serão definidas ainda nesse capítulo.

normalizadas, curvas como as sem quarks e sem glúons, sem $\tilde{g}$, mal apareceriam nos gráficos.

Espectros supersimétricos dependem, em princípio, dos particulares modelos de quebra soft de supersimetria. Porém o grande número de parâmetros supersimétricos confere uma grande liberdade na escolha desses espectros. O caso típico, contudo, é de forte hierarquia entre as partículas coloridas e não coloridas, ou seja, squarks e gluinos bem mais pesados do que sléptons, neutralinos, charginos, gauginos eletrofracos e Higgsinos. Ainda assim, dada a falta de evidência de nova física no LHC 7 e 8TeV, espectros de SUSY comprimidos tem sido cada vez mais estudados, uma vez que a degenerescência do espectro dificulta a observação de sinais com jatos e léptons duros.

Em nossos estudos, consideramos espectros com forte hierarquia entre squarks e gluinos, e o neutralino mais leve, e também alguns casos mais degenerados.

\subsection{Produção de KK-quarks e KK-glúons no LHC}

No modelo UED, a produção de partículas coloridas ocorre através de canais muito parecidos com os de supersimetria. Os squarks $(\tilde{q})$ de spin-0 são substituídos por KK-quarks $\left(q^{(1)}\right)$ de spin$1 / 2$, gluínos $(\tilde{g})$ de spin-1/2 por KK-glúons $\left(g^{(1)}\right)$ de spin-1 e o neutralino $\left(\tilde{\chi}_{1}\right)$ de spin-1/2 pelo primeiro estado de fóton excitado $\left(B^{(1)}\right)$ de spin-1. No caso de UED os KK-quarks apresentam-se como singletos $q^{(1)}$ S ou dubletos $q^{(1)}{ }_{D}$ do grupo gauge $S U(2)_{L}$.

Os espectros típicos de MUED são muito mais comprimidos do que os de supersimetria, variando em apenas algumas centenas de $\mathrm{GeV}$ desde o estado excitado mais leve, o fóton excitado, por exemplo, até o mais pesado, o glúon excitado [59], para uma dada escala de compactificação $1 / R$.

Ainda na produção de partículas oriundas de MUED, como dissemos anteriormente, numa realização típica desse modelo, os jatos terão menor momento transverso, assim como menos MET, devido à maior degenerescência do espectro, o que torna mais difícil separar estes eventos dos eventos do MP. Isso, naturalmente leva a uma impossibilidade de identificação do modelo correto baseado apenas no excesso de número de eventos. 


\subsection{Eventos de jatos + MET}

Entre outras características interessantes, como um mecanismo para manter a massa do bóson de Higgs em torno da escala eletrofraca, modelos supersimétricos e de dimensões extras universais ainda possuem candidatas naturais a matéria escura fria.

Como vimos anteriormente, os modelos de SUSY e MUED acomodam um candidato à matéria escura. Assim se a produção de partículas desses modelos ocorrer, como os squarks (KK-quarks) e gluinos (KK-glúons), teremos cadeias de decaimentos onde os produtos finais serão partículas de matéria escura e outras partículas estáveis do Modelo Padrão. Em nosso trabalho, consideramos apenas cadeias curtas de decaimento, onde gluinos decaem em squarks e quarks, e os squarks decaem diretamente em quarks e no neutralino (KK-fóton) dando origem a eventos de jatos e missing energy.

A presença de LSPs no estado final impede a reconstrução do 4-momento das partículas que originaram a cadeia de decaimento, mas é possível inferir a massa delas através de distribuições cinemáticas que exibem limiares [8, 9]. Isso, contudo, demanda uma boa reconstrução destas distribuições e isso só é possível com muitos eventos. Mais uma vez, isso adiciona evidência a um modelo ou outro, mas ainda não significa uma identificação positiva do modelo. Sem a possibilidade de identificar o modelo de nova física a partir do tamanho das seções de choque e do espectro, seja com eventos de jatos + MET, ou outra topologia, só resta o estudo das distribuições cinemáticas disponíveis.

Em cadeias longas, além das distribuições angulares, diferenças ou assimetrias entre distribuições de massas invariantes de conjuntos de partículas do estado final, como jatos e léptons carregados, podem ajudar a discernir entre modelos, veja [10, 11], por exemplo. Com cadeias curtas, como no decaimento direto de sbottoms [12] e de sleptons [13, 14], é possível estudar variáveis angulares correlacionadas com o ângulo de espalhamento do squark, ou slepton. Nesse trabalho, escolhemos trabalhar com as distribuições angulares de jatos produzidos no decaimento de squarks e gluinos aproveitando o número muito maior de eventos esperados em relação a sleptons ou na produção de sbottoms apenas.

\subsection{Produção dos espectros de nova física}

O espectro supersimétrico do MSSM necessita de escolhas arbitrárias de parâmetros, vide capítulo 1. Para qualquer modelo de supersimetria que assume um esquema de quebra em altas energias esses parâmetros podem ter sua evolução calculada através das equações do grupo de renormalização. No mSugra a evolução dos parâmetros é feita da escala Planck até a escala TeV. Já para o modelo GMSSB, a evolução é feita da escala de massa dos campos de gauge mensageiros até a escala $\mathrm{TeV}$.

Também temos modelos onde nenhum esquema de quebra supersimétrica é assumido, como o pMSSM, como vimos no capítulo 2, nesse modelo não há análise de parâmetros em altas energias. Porém, em qualquer caso de modelos supersimétricos, seja os modelos com quebra em altas escalas de energia ou no pMSSM [44], ainda existe uma liberdade de escolha dos parâmetros que definem o espectro de cada modelo.

Os modelos supersimétricos com quebra de supersimetria em altas energias têm fortes limites impostos pelo LHC hoje. Por exemplo, o modelo Gauge-Mediated para o MSSM possui bósons de gauge que comunicam a quebra de supersimetria para a escala $\mathrm{TeV}$, nesse modelo tem-se grandes dificuldades para conciliar um bóson de Higgs de $\sim 125 \mathrm{GeV}$ [72, 73], em sua versão mais simples, com massas das partículas supersimétricas abaixo de $2 \mathrm{TeV}$. Já o mSugra está muito desgastado pelos resultados recentes do LHC, os limites das seções de choque estão cada vez melhores, obrigando o mSugra a ter massas de partículas supersimétricas cada vez maiores, porém ainda é possível obter um escalar neutro de CP-par dentro desse modelo com massa de $125 \mathrm{GeV}$, é claro, assumindo uma certa quantidade de fine-tuning [74]. 
O nosso trabalho não é sensível a mecanismos particulares de quebra supersimétrica, o importante é a assinatura fenomenológica de um modelo supersimétrico na escala $\mathrm{TeV}$ com espectros ainda não excluídos pelo LHC. A relevância reside nos acoplamentos, topologia e spin das partículas envolvidas nos processos da QCD supersimétrica. Por isso implementamos um modelo simplificado em supersimetria para produção de squarks e gluinos. Esses modelos já são amplamente utilizados em análises fenomenológicas e experimentais [75, 90].

Os valores de massas para squarks e gluinos foram escolhidos apropriadamente para respeitar os limites impostos pelos mais recentes resultados do LHC de $8 \mathrm{TeV}$, com luminosidade integrada de $19.5 \mathrm{fb}^{-1}$ [49, 76], para modelos simplificados de supersimetria. Massas de squarks de sabores $\mathrm{u}, \mathrm{d}$, s e c têm valores não excluídos acima de $1 \mathrm{TeV}$ e massas de gluinos valores não excluídos acima de $1.3 \mathrm{TeV}$.

Em nosso modelo simplificado de supersimetria iremos estudar espectros, que em alguns casos, serão parecidos com os espectros típicos de mSugra. Aproveitando-se desse fato, usamos o programa SPheno. Esse programa tem implementado as equações do grupo de renormalização para o mSugra e definem o espectro na escala $\mathrm{TeV}$ a partir dos parâmetros de altas energias dados. O SPheno oferece rotinas que calculam automaticamente as larguras das partículas supersimétricas. Usando massas de squarks degenerados de $1.4 \mathrm{TeV}$, massa de gluino de $1.5 \mathrm{TeV}$ e massa de neutralino de $300 \mathrm{GeV}$ definimos as larguras através do SPHeno como as listadas na tabela 3.2 .

\begin{tabular}{|c|c|c|}
\hline & $\Gamma(\mathrm{GeV})$ & $\mathrm{m}(\mathrm{TeV})$ \\
\hline$\tilde{u}_{R}$ & 2.91 & 1.4 \\
$\tilde{u}_{L}$ & 13.21 & \\
\hline$\tilde{d}_{R}$ & 0.72 & \\
$\tilde{d}_{L}$ & 13.19 & 1.4 \\
\hline$\tilde{c}_{R}$ & 2.91 & \multirow{2}{*}{1.4} \\
$\tilde{c}_{L}$ & 13.21 & \\
\hline$\tilde{s}_{R}$ & 0.92 & \multirow{2}{*}{1.4} \\
$\tilde{s}_{L}$ & 13.19 & \\
\hline$\tilde{g}$ & 21.0 & 1.5 \\
\hline$\tilde{\chi}_{1}$ & - & 0.3 \\
\hline
\end{tabular}

Tabela 3.2: Um exemplo dentre os 150 espectros analisados em nosso trabalho, inspirado no modelo de mSugra. Os valores de massas listados ainda não foram excluídos pelo LHC, no contexto de modelos simplificados de supersimetria.

Para MUED necessita-se de 2 parâmetros para determinar todo seu espectro. O raio da dimensão extra $(R)$ e o cutt-off ultravioleta $\left(\Lambda_{U V}\right)$. Esses valores também são estudados baseados na descoberta do bóson de Higgs no LHC [64], temos limites impostos de $1 / R>1100 \mathrm{GeV}$ $\Lambda_{U V} R>20$ para os dados do LHC $8 \mathrm{TeV}$. Um raio de compactificação da ordem de TeV já alcança as massas de nosso espectro de referência para supersimetria.

O cenário mais difícil de discernimento entre supersimetria e dimensões extras universais é aquele em que as massas, as larguras e seções de choque são iguais em valores numéricos. Apesar de fictício, pois o modelo de MUED possui um espectro mais degenerado quando comparado ao dos modelos supersimétricos típicos, nesse regime o discernimento entre supersimetria e MUED é o mais complicado. Assim, assumimos uma postura maximamente conservadora quanto ao alcance do discernimento de modelos, a situação real deverá ser mais simples, então nosso limite estimado do alcance do LHC realmente testa o potencial do experimento de forma muito exigente. Sendo assim, apenas mudanças mais profundas entre os modelos, spin por exemplo, traria alguma pista sobre qual modelo apresenta melhor descrição dos dados experimentais.

A construção dos diversos espectros analisados em nosso estudo pode ser feita através das 
massas e larguras dos decaimento envolvidos na produção de jatos e MET. As larguras parciais de decaimento, a menos de acoplamentos, têm a seguinte dependência na massa dos squarks, gluinos e neutralinos [77, 78]:

- $\Gamma_{0}\left(\tilde{q} \rightarrow\right.$ jatos $\left.+\tilde{\chi}_{1}\right) \propto m_{\tilde{q}}\left(1-\left(\frac{m_{\tilde{\chi}_{1}}}{m_{\tilde{q}}}\right)^{2}\right)^{2}$.

- $\Gamma_{0}(\tilde{g} \rightarrow \tilde{q}+$ jatos $) \propto \frac{\left(m_{\tilde{g}}^{2}-m_{\tilde{q}}^{2}\right)^{2}}{m_{\tilde{g}}^{3}}$, se $m_{\tilde{g}}>m_{\tilde{q}}$.

- $\Gamma_{0}(\tilde{q} \rightarrow \tilde{g}+$ jatos $) \propto \frac{\left(m_{\tilde{q}}^{2}-m_{\tilde{g}}^{2}\right)^{2}}{m_{\tilde{q}}^{3}}$, se $m_{\tilde{g}}<m_{\tilde{q}}$.

As massas de quarks foram desprezadas. Em geral, decaimentos eletro-fracos de squarks em jatos e MET ocorrem através de duas partículas diferentes $\tilde{q}_{R}$ e $\tilde{q}_{L}$, a dependência das larguras de decaimento com as massas são idênticas para essas duas partículas, porém existe uma dependência da matriz de mistura dos neutralinos.

Construímos um espaço de massas em um plano $\left(m_{\tilde{q}}, m_{\tilde{g}}\right)$ que consiste em 150 pontos. Variamos a massa dos squarks de $1.4 \mathrm{TeV}$ até $5 \mathrm{TeV}$ em passos de $0.4 \mathrm{TeV}$, a massa dos gluínos de $2.0 \mathrm{TeV}$ a $5 \mathrm{TeV}$ em passos de $1 \mathrm{TeV}$, com a inclusão do ponto $1.5 \mathrm{TeV}$, além dos quatro já citados para os gluinos. E ainda três diferentes massas de neutralinos, $50 \mathrm{GeV}, 300 \mathrm{GeV}$ e $1 \mathrm{TeV}$. Pontos que possuem massas de squarks e gluínos idênticas, por exemplo $(3.0,3.0)$ e $(5.0,5.0)$, sofreram um aumento na massa dos gluínos de modo a evitar que sejam exatamente iguais, esses pontos se transformaram em $(3.0,3.1)$ e $(5.0,5.1)$.

O nosso espaço de massas contempla desde espectros mais comprimidos [44], com $\left|m_{\tilde{q}}-m_{\tilde{g}}\right| \geq$ $100 \mathrm{GeV}$ e $\left|m_{\tilde{q}}-\tilde{\chi}_{1}\right| \geq 400 \mathrm{GeV}$, até espectros fortemente hierarquizados típicos de SUSY, onde partículas coloridas são muito mais pesadas do que as não coloridas.

Definido o conjunto de massas e de larguras criam-se os arquivos necessários para que os programas MadGraph5, Pythia e PGS gerem os eventos para o LHC. Esses arquivos entram nos programas pelo nome de param_card.dat, são definidos no formato slha [79] para os eventos de supersimetria e slha2 [80] para os eventos de dimensões extras universais 1 .

Contabilizando larguras e massas temos 14 parâmetros a serem alterados para gerar 150 param_card.dat diferentes no formato slha e slha2. Isso totaliza de mais de 4200 mudanças em linhas de arquivos de texto. Trabalho tedioso, mas que pode ser minimizado a 6 segundos de cálculos de um computador modesto se usarmos algum programa para automação. Em nosso caso produzimos scripts escritos em PYTHON para realização desse trabalho.

A linguagem PYтноN oferece excelentes rotinas para manipulação de arquivos com uma biblioteca e desempenho poderosos, basicamente toda a manipulação de dados, testes, validações e integração de programas nesse trabalho foram realizadas em PYTHON.

\subsection{Canais para simulação de eventos}

\subsubsection{Supersimetria}

A produção de squarks (KK-quarks) e de gluinos (KK-glúons) e seu posterior decaimento em jatos e MET é um processo que depende da hierarquia de massas, como comentamos na seção 3.1 . Temos dois cenários: o primeiro $m_{\tilde{g}}>m_{\tilde{q}}$, aqui assumimos que o decaimento de gluinos (KKglúons) em squarks (kk-quarks) e quarks $(\tilde{g} \rightarrow \tilde{q} q)$ e o decaimento de squarks (kk-quarks) em jatos e neutralino (fóton pesado) ocorre com um branching ratio de 100\%. A contribuição vinda do canal de produção de pares de gluinos (KK-glúons) é muito pequena e pode ser desprezada,

\footnotetext{
${ }^{1}$ Supersymmetry Les Houches Accord.
} 
veja tabela (3.1). Simulamos, então, eventos para os seguintes canais:

$$
\begin{aligned}
& p p \rightarrow \tilde{q}_{i} \tilde{q}_{j}+(0,1) j \rightarrow j j+(0,1) j+\tilde{\chi}_{1}^{0} \tilde{\chi}_{1}^{0}, \\
& p p \rightarrow \tilde{q}_{i} \tilde{q}_{j}^{*}+(0,1) j \rightarrow j j+(0,1) j+\tilde{\chi}_{1}^{0} \tilde{\chi}_{1}^{0}, \\
& p p \rightarrow \tilde{q}_{i}^{*} \tilde{q}_{j}^{*}+(0,1) j \rightarrow j j+(0,1) j+\tilde{\chi}_{1}^{0} \tilde{\chi}_{1}^{0}, \\
& p p \rightarrow \tilde{q}_{i} \tilde{g} \rightarrow j j j+\tilde{\chi}_{1}^{0} \tilde{\chi}_{1}^{0} .
\end{aligned}
$$

Onde $q=(u, d, s, c)$ e $\{i, j\}=\{L, R\}$. No segundo cenário, na região $m_{\tilde{g}}<m_{\tilde{q}}$, canais com gluinos devem ser excluídos, portanto o sinal de supersimetria reduz-se a:

$$
\begin{aligned}
& p p \rightarrow \tilde{q}_{i} \tilde{q}_{j}+(0,1) j \rightarrow j j+(0,1) j+\tilde{\chi}_{1}^{0} \tilde{\chi}_{1}^{0}, \\
& p p \rightarrow \tilde{q}_{i} \tilde{q}_{j}^{*}+(0,1) j \rightarrow j j+(0,1) j+\tilde{\chi}_{1}^{0} \tilde{\chi}_{1}^{0}, \\
& p p \rightarrow \tilde{q}_{i}^{*} \tilde{q}_{j}^{*}+(0,1) j \rightarrow j j+(0,1) j+\tilde{\chi}_{1}^{0} \tilde{\chi}_{1}^{0} .
\end{aligned}
$$

Essa região tem contribuição em número de canais reduzida, mas mesmo assim, poderemos fazer alguma inferência sobre descoberta e discernimento de modelos de nova física, como veremos no capítulo Resultados.

Em 3.4 e 3.5), $j$ são jatos de todos os sabores de quarks leves e glúons. Analisamos somente quarks leves, porque o tagging de jatos de quarks e glúons é mais eficiente nessas partículas [21]. Além de que, a contribuição de maior relevância na seção de choque de produção de squarks vêm de gluinos no canal-t Fig. 3.2 e 3.5, na qual os quarks bottom pouco contribuem para colisões iniciais do tipo próton-próton. Novamente, retirar bottoms da simulação, torna nossa análise conservadora.

Incluímos a produção de jatos extras de radiação porque em processos de altas energia envolvendo QCD a produção desses jatos é abundante. Para isso ser feito devemos realizar a inclusão de processos independentes de produção de jatos, no final da simulação obtém-se dois conjuntos de simulação independentes, para que haja convergência no espaço de fase dessas duas simulações é necessário um processo denominado matching de jatos. Esse processo é feito automaticamente pelo MadGraph5 e Pythia, com os devidos ajustes que descreveremos ainda nesse capítulo.

\subsubsection{MUED}

Os eventos de MUED são produzidos a partir de canais de topologia idênticas aos de supersimetria. Para $m_{q^{(1)}}<m_{g^{(1)}}$ os canais que contribuem para o sinal de MUED são:

$$
\begin{aligned}
& p p \rightarrow q_{i}^{(1)} q_{j}^{(1)}+(0,1) j \rightarrow j j+(0,1) j+B^{(1)} B^{(1)}, \\
& p p \rightarrow q_{i}^{(1)} \bar{q}_{j}^{(1)}+(0,1) j \rightarrow j j+(0,1) j+B^{(1)} B^{(1)}, \\
& p p \rightarrow q_{i}^{(1)} g^{(1)} \rightarrow j j j+B^{(1)} B^{(1)} .
\end{aligned}
$$

Onde $q=(u, d, s, c)$. E os índices $i$ e $j$ agora referem-se a singletos (S) e dubletos (D).

E para $m_{q^{(1)}}>m_{g^{(1)}}$,

$$
\begin{aligned}
& p p \rightarrow q_{i}^{(1)} q_{j}^{(1)}+(0,1) j \rightarrow j j+(0,1) j+B^{(1)} B^{(1)}, \\
& p p \rightarrow q_{i}^{(1)} \bar{q}_{j}^{(1)}+(0,1) j \rightarrow j j+(0,1) j+B^{(1)} B^{(1)} .
\end{aligned}
$$

Algumas topologias de supersimetria não encontram equivalentes em MUED, como ilustrado na Fig. 3.6. O mesmo pode ser dito para supersimetria, pois vértices do tipo $g g \tilde{g} \tilde{g}$ não são permitidos, enquanto que para MUED $g g g^{(1)} g^{(1)}$ são permitidos.

Para MUED, assim como em supersimetria, processos com primeiro estado excitado de KKglúons no canal-t dominam a seção de choque de produção de KK-quarks. Por isso o discernimento entre supersimetria e MUED torna-se importante, usando informações de diferentes 

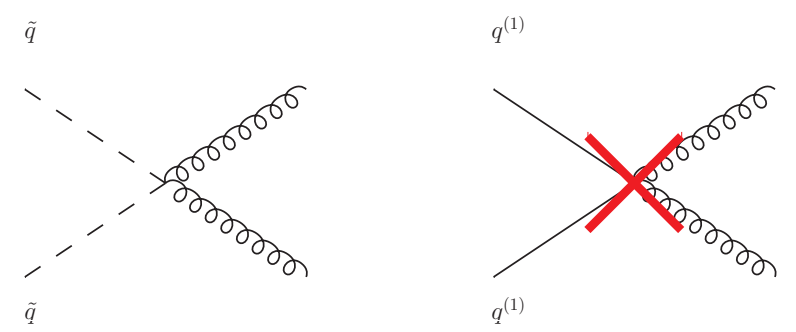

Figura 3.6: Vértices que não possuem topologia equivalente em supersimetria e MUED.

números quânticos presentes em cada hipótese, que por sua vez estão contidas em observáveis físicos apropriados, é possível dizer se um modelo é favorecido com relação a outro. Embora a topologia para produção de jatos e MET seja, efetivamente, idêntica nas duas hipóteses.

\subsubsection{Backgrounds}

Os backgrounds para jatos e MET são definidos em duas grandes categorias, redutíveis e irredutíveis. Os backgrounds irredutíveis são aqueles que contribuem diretamente para imitar o sinal de jatos e MET, esses eventos possuem o mesmo número de partículas no estado final, após cortes, aceitação e seleção de eventos. Já os background redutíveis, são os eventos que não possuem a mesma topologia de estado final, mas eventualmente, por alguma característica de detecção, acabam sendo confundidos como sinal.

Para produção de jatos e MET, os backgrounds irredutíveis são,

$$
\begin{aligned}
& p p \rightarrow q q+\{Z \rightarrow \nu \bar{\nu}\}+(0,1) j \rightarrow j j+(0,1) j+\nu \bar{\nu}, \\
& p p \rightarrow q g+\{Z \rightarrow \nu \bar{\nu}\}+(0,1) j \rightarrow j j+(0,1) j+\nu \bar{\nu}, \\
& p p \rightarrow g g+\{Z \rightarrow \nu \bar{\nu}\}+(0,1) j \rightarrow j j+(0,1) j+\nu \bar{\nu} .
\end{aligned}
$$

Esses processos têm a maior contribuição em seção de choque de todos os processos referentes aos backgrounds. Nesses canais também simulamos produção de jatos extras de QCD.

Para os backgrounds redutíveis para jatos e MET temos:

$$
\begin{aligned}
& p p \rightarrow q q+\left\{W^{ \pm} \rightarrow l^{ \pm} \nu_{l}\right\}+(0,1) j \rightarrow j j+(0,1) j+\nu l^{ \pm}, \\
& p p \rightarrow q g+\left\{W^{ \pm} \rightarrow l^{ \pm} \nu_{l}\right\}+(0,1) j \rightarrow j j+(0,1) j+\nu l^{ \pm}, \\
& p p \rightarrow g g+\left\{W^{ \pm} \rightarrow l^{ \pm} \nu_{l}\right\}+(0,1) j \rightarrow j j+(0,1) j+\nu l^{ \pm} .
\end{aligned}
$$

O lépton adicional no estado final torna o processo completamente diferente de dois jatos e neutrinos somente, em princípio. Porém, como a seção de choque de produção de $W+$ jatos é grande, a fração de eventos em que estes léptons não sejam detectados ou por serem muito moles (baixo momento transverso) ou por atingirem regiões periféricas do detector (grande rapidez), ou ainda por não estarem suficientemente isolados de jatos, por exemplo, ainda constitui um background não desprezível. A boa notícia é que esses eventos não sobrevivem a cortes de $H_{T}$, $\mathbb{F}_{T}$ e $p_{T}$ acima de $500 \mathrm{GeV}$. Como veremos, cortes bem mais duros do que estes serão aplicados para separar os eventos de sinal.

Também podemos ter a produção pura de jatos vindos de QCD como um background redutível. Os processos responsáveis por produção pura de QCD são:

$$
\begin{aligned}
& p p \rightarrow j j, \\
& p p \rightarrow j j j, \\
& p p \rightarrow j j j j .
\end{aligned}
$$

No processo de hadronização, alguns jatos vindos de quarks têm baixa energia e isso torna a identificação dos hádrons que o compõe mais difícil. Assim eventos de 2 ou mais jatos são 
confundidos com eventos de menos jatos, levando a um desbalanço de MET. Já quando o jato é muito energético, a produção de hádrons é mais colimada e a identificação é mais eficiente, diminuindo significativamente o MET associado. Cortes acima de $200 \mathrm{GeV}$ em MET retiram eventos vindos desse background.

Ainda para os backgrounds redutíveis temos a produção de quarks tops,

$$
p p \rightarrow t \bar{t} \rightarrow b+\{W \rightarrow j j\}+\bar{b}+\{W \rightarrow l \nu\} .
$$

Aqui a perda de um lépton pode levar a eventos com jatos + MET no estado final. Verificamos também que esse processo não passa nos cortes retangulares usados no resultado final.

\subsection{Simulação de eventos}

O programa usado para geração dos eventos de nosso trabalho é o MADGraPh5 [81, versão 2.2.3. Trata-se de um programa para produção dos diagramas de Feynman relacionados a um dado processo para um dado modelo de física de partículas em altas energias, além de realizar a integração, automática, do espaço de fase necessário para a geração dos eventos. É um programa baseado em integrações através de processos de Monte Carlo.

O MadGraph5 acompanha por padrão uma vasta variedade de modelos. Dentre eles o MSSM, modelo usado nessa análise para simular os eventos de supersimetria com os devidos ajustes. Infelizmente a versão MADGraph5 2.2.3 ainda não acompanha o modelo de MUED. Mas o addon para Mathematica chamado FeynRules traz ferramentas para criação e alguns modelos prontos para uso no MadGraph5 2 E com o FeynRules criamos o modelo efetivo de MUED usado nesse trabalho.

O programa MadGraph5 será associado com os programas Pythia [82] e PGS [83], responsáveis pela hadronização e parton shower e eficiência de deteç̧ão, respectivamente. O MADGRAPH5 tem a maioria dos seus parâmetros ajustados por um arquivo chamado run_card.dat, nele escolhemos o tipo de acelerador da simulação, a energia das colisões, o número de eventos, escalas de fatorização, renormalização, matching dentre outros.

O número de eventos gerados para nossa análise foi 45.000 para cada canal de supersimetria e MUED. Esse valor foi escolhido baseado na prescrição que os desenvolvedores do MADGRAPH5 recomendam para uma melhor integração com o programa PyтнiA.

Os próximos parâmetros que ajustamos foram a escala de fatorização e renormalização. Aqui cada canal tanto de sinal e background precisam ter escolhas diferentes. Pois a nível de árvore as seções de choque tendem a ter uma dependência grande desses fatores, por isso precisam ser escolhidos apropriadamente. Para o sinal utilizamos a convenção dada em [84], onde a escala é escolhida de acordo com a média das massas das partículas produzidas no estado intermediário. Para produção de squarks a escala de fatorização e renormalização é $\frac{m_{\tilde{q}_{1}}+m_{\tilde{q}_{2}}}{2}$, para produção de squarks e gluinos a escala é $\frac{m_{\tilde{q}}+m_{\tilde{g}}}{2}$, a mesma convenção é adotada para os canais de produção de kk-quarks e kk-glúons.

Para os backgrounds fizemos uma escolha de escala dinâmica. O valor da escala de fatorização é escolhido de acordo com as configurações de cada evento. Isso pode ser feito no MadGraph5. É possível escolher uma escala $\left(\mu^{2}\right)$ que dependa da soma de momentos transversos e massa invariante para cada partícula em um dado evento,

$$
\mu^{2}=\sum_{i}\left(p_{T_{i}}^{2}+m_{i}^{2}\right)
$$

onde a soma $i$ é realizada sobre o número de eventos, e $m_{i}^{2}=\vec{p}_{i} \cdot \vec{p}_{i}$. Fizemos essa escolha para os backgrounds pois não temos uma grande criação de partículas intermediárias, já que seus jatos

\footnotetext{
${ }^{2}$ https://cp3.irmp.ucl.ac.be/projects/madgraph/wiki/Models [17/05/2015].
} 
vêm basicamente de radiação, por isso convenciona-se uma escolha alternativa para fixar o valor das escalas.

A geração de eventos para o MAdGRAPH5, com produção extra de partons, ainda requer um corte em $p_{T}$ de $20 \mathrm{GeV}$, para retirada de partons que eventualmente podem ter energias muito baixas e a convergência dos diagramas de Feynman ficam prejudicadas, a nível de árvore. Falaremos mais sobre isso na próxima seção. A geração de eventos também possui um corte máximo para rapidez dos jatos, $|\eta|<5$.

\subsubsection{Matching de jatos}

A produção inclusiva de eventos, realizada em nossa análise, exige a geração de jatos vindos de radiação de QCD. O programa PyTHIA será o responsável pelo parton shower (PS) e hadronização dos eventos. Porém, em nosso caso, poderemos produzir jatos muito energéticos e bem separados no detector, um regime onde o Pyтнia não possui boa convergência. Assim precisamos ajustar alguns parâmetros para o correto tratamento da região de transição entre os regimes soft e hard de emissão de partons extras [85]. Seja qual for o gerador de eventos utilizado, combinar as regiões de emissão soft dos Parton Showers e emissão hard com elementos de matriz (ME) exatos é uma tarefa imprescindível para obter resultados consistentes.

A integração no ME + PS dá origem a um problema de superposição de espaço de fases quando uma análise com inclusão de partons extras é realizada. Por exemplo, em uma análise inclusiva como a nossa onde amostras de 0 e 1 parton extra são adicionadas em processos diferentes, pode ocorrer que após o PS, uma amostra de 0 parton já tenha sido considerada pelo ME nos eventos gerados com 1 parton extra. Isso é a chamada superposição de espaço de fases. Para evitar isso o matching de jatos é necessário.

O processo ocorre da seguinte maneira para o esquema $k_{T}$ MLM [86]. Partons no estado final dos eventos gerados pelo MADGraph5 são agrupados segundo um algoritmo $k_{T}$, somente agrupamentos que possuem uma correspondência com o que foi gerado pelo ME são mantidos na simulação. O esquema $k_{T}$ necessita de um parâmetro para agrupamento dos jatos, no MADGraph5 é chamado de xqcut. Logo após o Pythia inicia o PS, antes da hadronização os novos partons finais serão agrupados em jatos usando o mesmo esquema $k_{T}$, a diferença está na escala de agrupamento, que será agora qcut. Esses novos jatos serão comparados com os originais do ME, um jato terá correspondência com o parton inicial se $k_{t}$ (parton, jato) < qcut. Se isso ocorrer o evento é mantido na simulação, caso contrário é excluído. Na maioria das vezes, os eventos que não conseguem ter uma correspondência são aqueles que os partons estão próximos, de modo que são confundidos como sendo apenas 1 jato. Ou quando os partons tem energia baixa e não conseguem gerar seu próprio jato.

A eficiência de matching de jatos, em nosso caso, está por volta de $50 \%$, para sinal e background. Espectros mais pesados de supersimetria e MUED tendem a ter eficiências melhores, justamente por produzirem partons mais separados e mais energéticos.

Temos na Fig: 3.7 um exemplo prático da correspondência de jatos em nossa análise. Foi esboçado somente uma parte de um dos diagramas que contribuem para a produção de sinal de supersimetria. A produção de squarks com 1 jato extra é feita no Matrix Element, quando o Pyтнia começa o PS, acaba ocorrendo a geração de eventos que já foram incluídos na simulação. O matching exclui eventos que não possuem uma correspondência partônica com o ME, garantindo assim que o PS não inclua eventos que já foram contabilizados pelo ME (double counting). Vale ressaltar que uma má escolha dessas escalas leva a uma contagem de jatos errada.

As escalas de matching (xqcut e qcut), para os jatos, necessitam de uma análise individual para cada canal. Não existe uma regra geral para qual valor usar, é um processo de tentativa e erro. A sugestão é que xqcut tenha uma valor entre $1 / 6$ a $1 / 2$ da escala de fatorizaçãorenormalização. Felizmente o MADGraph5 tem um teste para verificar se a escolha de escala foi apropriada, são as figuras para diferential jet rate (DJR). Se essas distribuições estiverem contínuas então as escolhas de matching foram adequadas. A escolha de escala soft que usamos 


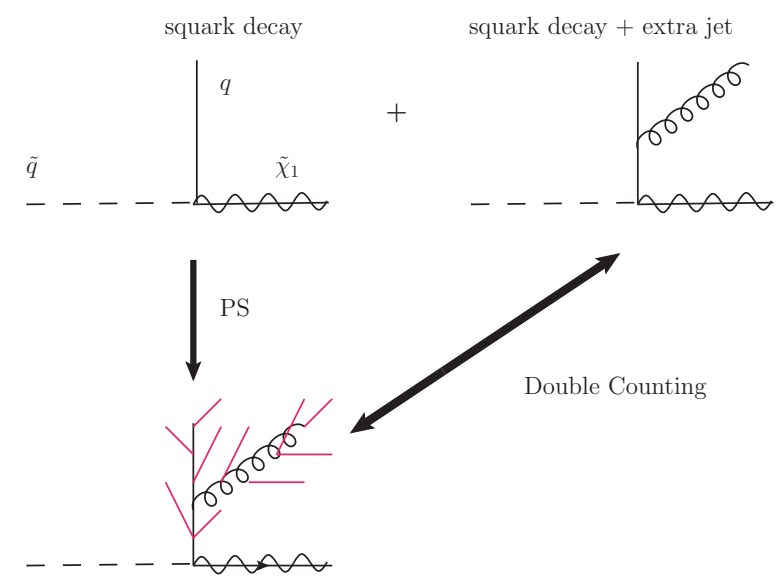

Figura 3.7: Exemplo de como o Pутніл insere processos (parte inferior da figura) que já foram calculados pelo MADGRAPH5 na geração de eventos com inclusão de jato extra (parte superior da figura).

foi a descrita na tabela 3.3 .

\begin{tabular}{|c|c|c|}
\hline & xqcut $(\mathrm{GeV})$ & qcut $(\mathrm{GeV})$ \\
\hline susy & 100 & 120 \\
mued & 100 & 120 \\
bckg & 10 & 15 \\
\hline
\end{tabular}

Tabela 3.3: Valores da escala de correspondência para MadGraph5 e Pythia.

Na Fig. 3.8 temos um exemplo da distribuição DJR1 para uma simulação de supersimetria. Essa distribuição não possui um significado físico, pois ela depende de escalas de correspondência inseridas apenas para evitar sobreposição de dois espaços de fases. DJR1 denota a distância (escala) de transição de uma amostra de 1 jato para uma de 0 jato. Se essa distribuição não for contínua aconteceu algum problema no matching de jatos. Aliás, quando essa distribuição não é contínua, a distribuição de $p_{T}$ dos jatos no estado final também apresenta descontinuidades, denotando que algumas regiões do espaço de fase acabaram com menos eventos de jatos do que outras. Em nosso trabalho temos 900 canais de simulação e verificamos para todos eles que essas distribuições são contínuas.

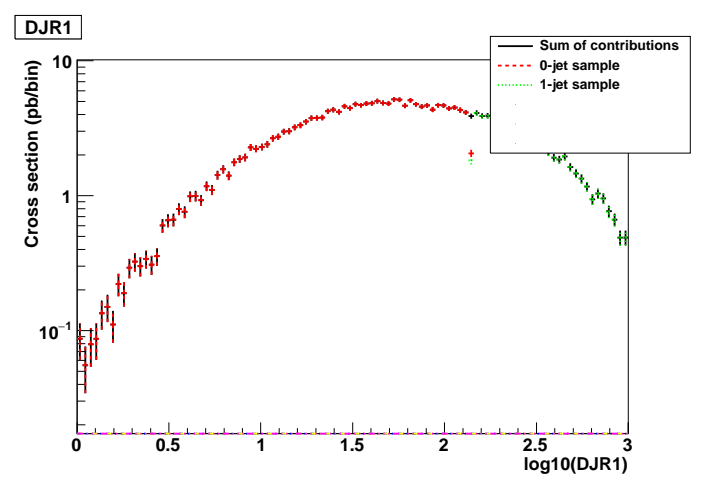

Figura 3.8: Distribuição para avaliação da escolha de escala para correspondência de jatos.

Alguns ajustes ainda são necessários para a realização do matching. A variável,

$$
T=\text { auto_ptj_mjj }
$$


garante uma escolha automática para cortes em $p_{T}$ e massas invariantes dos jatos no mesmo valor de xqcut, fundamental para o uso do esquema $k_{T}$ MLM. Deve-se também estar atento aos cortes em drjj, versões mais novas do MADGRAPH5 ajustam esse corte automaticamente para zero quando uma simulação com matching é realizada.

\subsubsection{Subtração de gluinos ressonantes}

A inclusão de jatos extras em uma análise inclusiva necessita ainda da subtração de ressonâncias de gluinos no canal de produção de squarks. No processo $p p \rightarrow \tilde{q} \tilde{q}+j$, o MADGraph5 inclui diagramas que também são contabilizados no processo $p p \rightarrow \tilde{q} \tilde{g}$, gerando um outro double-counting, veja a Fig. 3.9. Esse problema aparece no momento de produzir jatos extras na produção de squarks, pois o MADGRAPH5 produz alguns dos processos calculados em $p p \rightarrow\left(\tilde{q} \rightarrow j \tilde{\chi}_{1}\right)+\left(\tilde{g} \rightarrow j j \tilde{\chi}_{1}\right)$ para o canal $p p \rightarrow \tilde{q} \tilde{q}+j$. Como a nossa análise é inclusiva e leva em consideração esses dois canais, isso é um problema. A solução desse problema é subtrair as ressonâncias de gluinos dos diagramas de produção de squarks com jatos extras. Pode-se realizar isso tanto a nível de elementos de matriz (MADGraPh5), quanto à nível de parton shower (Pythia). Escolhemos a implementação através do Pythia, que é realizada através do comando EXCRESS.
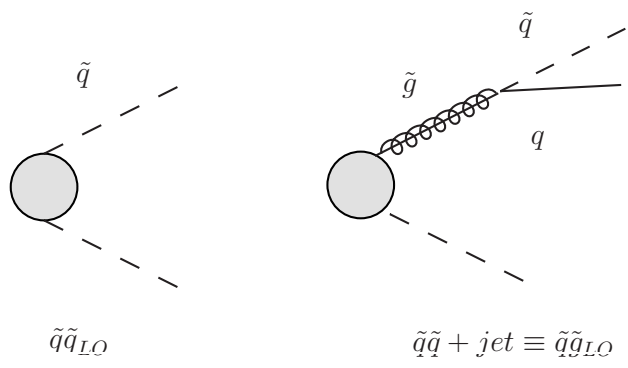

Figura 3.9: Exemplo de como o MADGraph5 introduz diagramas repetidos em processos que possuem ressonâncias de partículas nos estados intermediários, para a simulação de eventos com jatos e MET através da produção dos canais $\tilde{q} \tilde{q}$ e $\tilde{q} \tilde{g}$.

\subsubsection{PGS}

O programa PGS [83] é um simulador rápido de efeitos de detectores para processos de física de altas energias. Esse programa incorpora um conjunto de rotinas para eficiências de detecção parametrizando a resposta do detector em função das propriedades dos jatos, léptons, fótons e missing energy. É possível escolher diversos detectores pré-configurados. Em nosso caso escolhemos o ATLAS. Não deve-se esperar grandes diferenças em relação ao CMS de qualquer forma.

\subsection{Seleção de eventos}

As variáveis usadas na seleção de eventos do nosso trabalho são as mais exploradas em buscas de supersimetria no LHC, para eventos com jatos e missing energy $-H_{T}, \mathbb{H}_{T}$ e $p_{T}$ de jatos.

A Fig. 3.10c mostra que o $p_{T}$ de jatos é uma boa variável para separação entre sinal e background em eventos de produção de jatos e MET. Selecionaremos eventos com dois jatos com alto $p_{T}$, isso porque a topologia de produção de squarks (kk-quarks) privilegia a produção de pares de jatos provenientes de decaimento direto de partículas pesadas. 
A variável $H_{T}$ é definida como:

$$
H_{T}=\sum_{i}^{N} p_{T i},
$$

onde $N$ é o número total de partículas no estado final de cada evento. Aqui usamos novamente o $p_{T}$, mas levando em conta o momento transverso de todas as partículas do estado final. Isso vai garantir uma separação de sinal e background ainda maior, pois serão poucos os eventos de backgrounds que terão um momento transverso grande para 1,2 ou 3 jatos. Com $H_{T}$, garantimos que muitos jatos com alto $p_{T}$ serão selecionados. Diminuindo ainda mais os eventos para os backgrounds.

Como podemos ver na Fig. 3.10, os backgrounds possuem valores médios, para cada uma das distribuições apresentadas, abaixo dos valores médios de supersimetria. Justamente por isso essas variáveis são interessantes na seleção de eventos de nova física para jatos e MET no estado final.

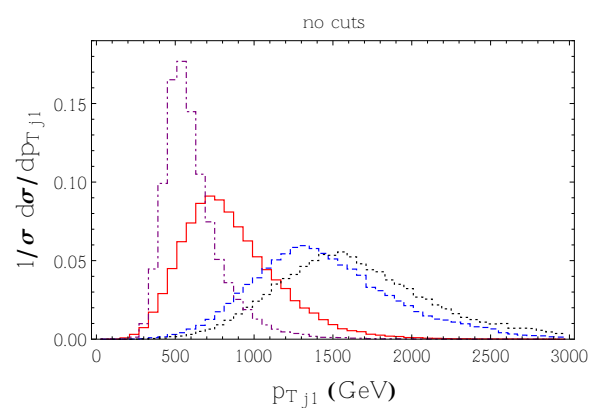

(a)

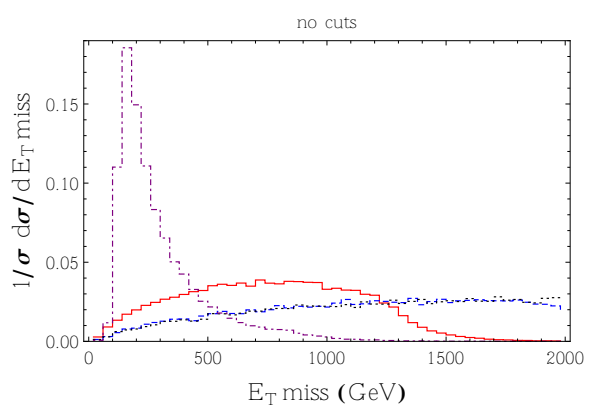

(b)

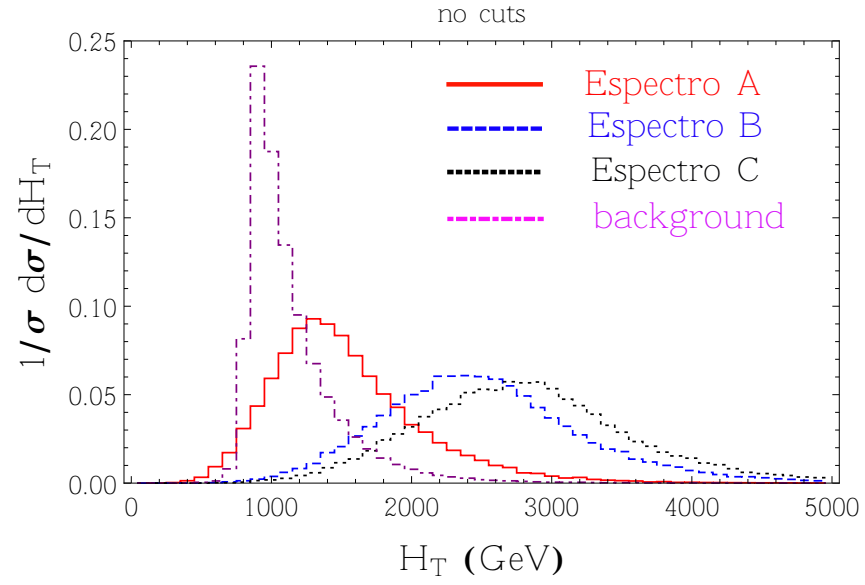

(c)

Figura 3.10: Distribuições normalizadas para as variáveis usadas na separação de eventos de sinal total e background total.

Os cortes em $p_{T}$ serão aplicados para os dois jatos mais duros de cada evento. Os jatos vindos do decaimento direto de squarks serão aqueles com maior $p_{T}$, jatos de radiação de QCD não conseguem ser tão energéticos quanto eles.

As variáveis $\mathbb{H}_{T}$ e $H_{T}$ têm valores médios baixos para os backgrounds. Mas a seção de choque para esses eventos chega a ser milhões de vezes maior do que as de supersimetria. Portanto, os cortes serão maiores que $1 \mathrm{TeV}$ para removermos razoavelmente os backgrounds. E por essa razão, o número de eventos para os backgrounds têm uma grande diminuição com cortes em $H_{T}$, $\mathscr{H}_{T}$ e $p_{T}$ altos. A fração de eventos que passam nesses cortes são, em média, $10 \%$ para $\not_{T}$ e $H_{T}>1 \mathrm{TeV} \operatorname{com} p_{T}>400 \mathrm{GeV}$. 
Selecionamos eventos também com a variável pseudo-rapidez $(\eta)$,

$$
\left|\eta_{j}\right|<2.5
$$

Para os dois jatos mais duros de cada evento. O valor de 2.5 é escolhido pois os jatos potencialmente de nova física serão os de decaimento de squarks pesados, altamente energéticos e portando com rapidez nesse intervalo.

\subsection{Observáveis físicos para jatos e MET}

O likelihood binado permite construir PDFs conjuntas que podem conter informações de diversos observáveis físicos diferentes. Inserir mais observáveis é um processo que tende a melhorar um teste estatístico construído com uma função likelihood, desde que o observável escolhido seja capaz de ter algum poder de discernimento entre os modelos analisados. Escolhemos um conjunto de 9 variáveis (observáveis ou distribuições) sensíveis, em algum nível, ao spin das partículas intermediárias dos modelos de supersimetria e MUED. Como discutido anteriormente, os dois jatos mais energéticos serão os jatos que potencialmente terão informações de nova física, definiremos observáveis que são funções dos dois jatos mais duros de cada evento, denotaremos por $\left(j_{1}, j_{2}\right)$, $p_{T_{1}}>p_{T_{2}}$.

As distribuições de nossa análise dependem de $p_{T}$, massas invariantes e rapidez dos jatos mais energéticos de cada evento.

A rapidez é definida como:

$$
y=\frac{1}{2} \log \left(\frac{E+p_{z}}{E-p_{z}}\right)
$$

A diferença de rapidez é uma medida invariante sobre boosts ao longo do eixo $z$ de colisão dos hádrons, o que a torna interessante em aceleradores do tipo do LHC, pois a natureza composta dos prótons torna difícil determinar o referencial onde a colisão de cada parton ocorreu. Para definir a rapidez (3.16) são necessárias informações como o momento ao longo eixo $z$, medida complicada no LHC pois o feixe de colisão atrapalha uma medida precisa do momento nessa direção, e a energia da partícula. A saída é usar a pseudo-rapidez, $\eta=-\log \tan \frac{\theta}{2}$. Ela é definida a partir da rapidez $y$ no regime de altas energias. Diferenças de pseudo-rapidez também são invariantes sobre boosts no eixo $z$, e a medida de $\eta$ depende somente do ângulo da partícula com relação ao feixe do acelerador, $\theta$.

As distribuições escolhidas mostraram-se boas para aumentar a capacidade de descoberta e discernimento. Elas oferecem a vantagem de ser adimensionais. Pode-se mostrar que variáveis adimensionais, em um decaimento de uma partícula visível e outra invisível, independem da massa da partícula invisível, desde que os observáveis construídos dependam apenas dos momentos da partícula visível [14]. E notamos que elas oferecem um ganho no poder de descoberta e discernimento de nova física. Além disso essas distribuições, em geral, estão relacionadas com momentos transversos de jatos muito energéticos e massas transversas, garantindo também que são invariantes sobre boosts ao longo do eixo z. As distribuições cinemáticas usadas foram:

$\cos \theta_{\mathbf{B}}$

Analisando decaimentos altamente energéticos de partículas de nova física em jatos ou léptons e MET, mostrou-se [13, 14] que é possível diferenciar modelos com partículas $Y$ de diferentes estatísticas ou spin, em um regime de altas energias. A partícula $Y$ pode ser um slépton-squarks (spin-0) ou KK-lépton-KK-quarks (spin-1/2). Como exemplo veja a Fig. 3.11 .

A seção de choque de produção para as partículas $Y$ e $\bar{Y}$ com massas $\mathcal{O}(T e V)$, vindas de uma contribuição do tipo canal-s, em função do ângulo entre um parton inicial e a partícula 


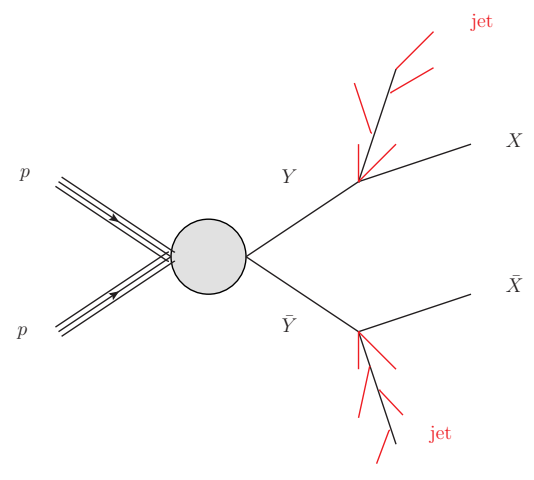

Figura 3.11: Possível topologia para $p p \rightarrow Y \bar{Y} \rightarrow j X+j \bar{X}$.

$Y\left(\theta^{\prime}\right)$, para o referencial do centro de massa de $Y \bar{Y}$ é dada para supersimetria da seguinte forma:

$$
\frac{d \sigma}{d \cos \theta^{\prime}} \propto 1-\cos ^{2} \theta^{\prime}
$$

E para MUED,

$$
\frac{d \sigma}{d \cos \theta^{\prime}} \propto 1+\frac{E_{Y}^{2}-m_{Y}^{2}}{E_{Y}^{2}+m_{Y}^{2}} \cos ^{2} \theta^{\prime}
$$

onde $E_{Y}$ e $m_{Y}$ são a energia e massa do KK-quark, no referencial de centro de massa.

A produção de partículas $Y$ e $\bar{Y}$ será feita com ângulos diferentes para cada modelo, isso é um mecanismo de determinação do spin dessas partículas nessa topologia de produção via canal-s. Quando os sléptons/squarks (KK-léptons/KK-quarks) decaírem, devido à sua grande massa, iremos observar que léptons/quarks altamente energéticos, e portanto com altos boosts no referencial de laboratório ${ }^{3}$ Para supersimetria teremos léptons/quarks, em média, menos espalhados em $\theta^{\prime}$ do que os léptons/quarks de MUED.

Mostrou-se [13] que o ângulo polar $\theta^{\prime}$ possui uma correlação no regime de altas energias com a diferença de pseudo-rapidez dos léptons do estado final no referencial de centro de massa dos próprios sléptons e kk-léptons produzidos. E que esse ângulo no decaimento dos sléptons em léptons através da produção de matéria escura independe da massa da matéria escura.

Nosso trabalho faz uso de jatos ao invés de léptons, e isso traz um desafio a mais. Ainda é possível definir um ângulo polar para jatos com analogia ao que foi feito para os léptons [12. Esse ângulo polar estará correlacionado também com a diferença de pseudo-rapidez dos jatos no estado final no regime de altas energias. Porém para supersimetria e MUED a topologia dominante na produção de jatos e $\mathbb{F}_{T}$ é o canal-t com gluinos (kk-glúons). Além do que fatores como parton shower, hadronização, efeitos de detector e cortes retangulares influenciam de maneira relevante no poder de correlação para o caso, de por exemplo, $Y$ sendo sléptons (kk-léptons).

A variável que possui uma correlação de spin em regimes de altas energias, independe da massa das partículas envolvidas e possui invariância sob boosts ao longo do eixo-z é:

$$
\cos \theta_{B}=\tanh \left(\frac{\Delta \eta_{i j}}{2}\right)
$$

\footnotetext{
${ }^{3}$ Ressaltamos que realizações típicas de MUED não produzem jatos duros em seus decaimentos, pois seu espectro é mais comprimido do que os típicos de supersimetria. Porém, para espectros de MUED normalizados aos de supersimetria, teremos um favorecimento à produção de jatos duros em ambos os modelos.
} 
onde $\eta_{i}$ é a pseudo-rapidez de uma dado jato medida no referencial do laboratório.

Eventos com produção de MET no LHC não fornecem a possibilidade de reconstrução do ângulo de espalhamento dos squarks ou kk-quarks. A variável (3.19) vem suprir essa necessidade. Na Fig. 3.12 temos a distribuição cinemática de (3.19) para ilustração. Aqui é um caso onde não foi incluído parton shower, hadronização e eficiência de deteç̧ão.

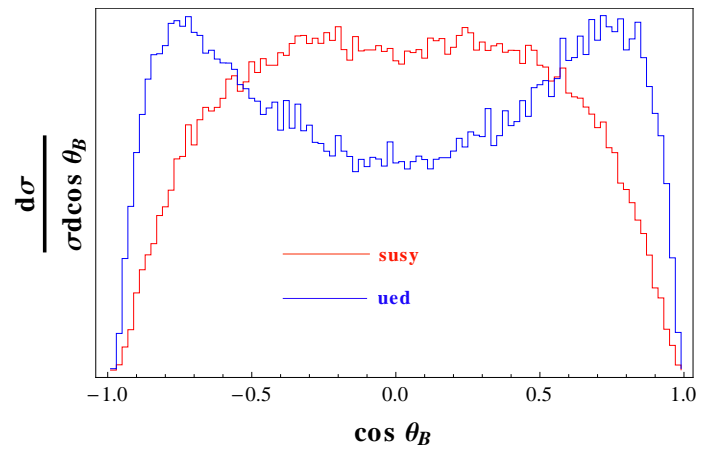

Figura 3.12: Um exemplo de distribuição cinemática usada em nosso trabalho, perceba que para cada um dos modelos temos distribuições com formas diferentes. O modelo de supersimetria tem produção de quarks menos separados do que o modelo de MUED, no referencial do laboratório.

$\cos \theta_{\mathbf{M}}$

Essa variável é definida de maneira análoga ao $\cos \theta_{B}$, com a vantagem de ser mais sensível ao spin de partículas intermediárias quando comparada $\operatorname{com} \cos \theta_{B}$ segundo [14]. $\cos \theta_{M}$ será o cosseno do ângulo de espalhamento dos jatos e o feixe do acelerador no referencial do centro de massa dos jatos visíveis. Basicamente temos que somar os momentos dos dois jatos mais duros $\left(P=p_{i}+p_{j}\right)$, calcular sua massa invariante $\left(m_{i j}=\sqrt{P^{2}}\right)$ e fazer um boost do momento dos jatos para o referencial onde $Q=\left(m_{i j}, 0,0,0\right)$, o ângulo $\theta_{M}$ é definido nesse referencial. $\cos \theta_{B}$ e $\cos \theta_{M}$ coincidem em regime de altas energias no caso onde $p_{i}=-p_{j}$.

A variável $\cos \theta_{M}$ não depende da pseudo-rapidez, em contraste à variável $\cos \theta_{B}$. Com isso $\cos \theta_{M}$ não apresenta um efeito de smearing devido à eficiência de deteç̧ão com relação à pseudo-rapidez.

$\alpha_{\mathbf{T}}$

Essa variável adimensional foi proposta inicialmente em [87] como uma variável sensível a diferentes modelos de nova física. Ela é definida como:

$$
\alpha_{R}=\frac{p_{T j_{1}}}{m_{j_{1} j_{2}}}
$$

onde $p_{T i}$ é o momento transverso do jato mais energético e $m_{j_{1} j_{2}}$ é a massa invariante dos dois jatos mais energéticos de um dado evento.

$\cos \theta^{*}$

Variável responsável pela medida do ângulo que o jato mais energético faz com o eixo $z$ positivo.

$\cos \theta_{\mathbf{i j}}$

Defini-se primeiramente os dois jatos mais duros de momento $\vec{p}_{1}$ e $\vec{p}_{2}$, onde $p_{1}^{\mu}$ é o mais duro. Então devemos fazer um boost do momento $p_{1}$ para o referencial de repouso de $p_{1}+p_{2}$. Assim $\theta_{i j}$ será o ângulo entre $\overrightarrow{p_{1}}$ boosted e o vetor $\overrightarrow{p_{1}}+\overrightarrow{p_{2}}$ no referencial de laboratório. 
$\Delta \mathrm{R}_{\mathrm{ij}}$

Com essa variável medimos a distância em um plano cartesiano de coordenadas $(\eta, \phi)$ entre os dois jatos mais duros.

$$
\Delta R=\sqrt{\delta \eta^{2}+\delta \phi^{2}}
$$

$\eta$ é a pseudo-rapidez e $\phi$ é o ângulo entre o $p_{T}$ da partícula e o eixo $z$.

$\Delta \phi_{\mathbf{i j}}$

Separação angular $(\phi)$ entre os dois jatos mais duros.

$\chi_{\mathbf{T}}$

Essa variável é definida em analogia com o $\alpha_{R}$, mas agora ao invés de usar a massa invariante $m_{j_{1} j_{2}}$ usa-se a massa $M_{T 2}$ dada por [88, 8]:

$$
\begin{gathered}
\chi_{T}=\frac{p_{T j_{1}}}{M_{T 2}} . \\
M_{T 2}=\min _{q_{1}+q_{2}=p_{T}} \max \left\{m_{T}\left(p_{1}, q_{1}, \chi\right), m_{T}\left(p_{1}, q_{1}, \chi\right)\right\},
\end{gathered}
$$

onde $m_{T} \equiv$ função de massa tranversa é dada por:

$$
m_{T}(p, q, \chi)=m_{p}^{2}+m_{\chi}^{2}+2\left(E_{T}^{p} E_{T}^{q}-\vec{p} \cdot \vec{q}\right)
$$

Aqui $p_{1}$ e $p_{2}$ são os momentos das partículas visíveis e $p_{T}$ o momento transverso faltante. É necessário fazer uma suposição sobre o valor de massa da matéria escura $m_{\chi}$, mas essa escolha não influencia fortemente o resultado final [89].

$\mathbf{N}_{\text {jets }}$

Trata-se do número de jatos gerados no estado final de cada evento.

Na Fig. 3.13 temos nove figuras para as distribuições dos três modelos estudados (supersimetria, MUED e o Modelo Padrão). Os cortes aplicados a essas figuras são somente os relacionados à geração dos eventos, veja a seção 3.7. As mudanças na forma das distribuições é o que a análise multivariada leva em conta, quanto maior as diferenças entre os formatos das distribuições, melhor é o poder de discernimento de modelos na análise multivariada. As seções de choque de produção de backgrounds, para o Espectro A, superam a seção de choque de produção de supersimetria em um fator de 15 nesse caso. Quando aplicarmos os cortes retangulares para remover os backgrounds e aumentar o poder de descoberta a seção de choque e o formato de todas distribuições mudam, tornando o formato das distribuições mais parecidos.

Na Fig. 3.14, temos novamente as nove distribuições normalizadas. Agora variamos os espectros de massa, mantendo $m_{\tilde{\chi}_{1}}=m_{B^{(1)}}=300 \mathrm{GeV}$, com massas de squarks e gluinos $\left(m_{\tilde{q}}, m_{\tilde{g}}\right)$ de $(1.4,1.5),(2.6,3.0)$ e $(3.0,4.0) \mathrm{TeV}$. As distribuições com curvas sólidas (supersimetria) tendem a ser parecidas na forma com outras curvas sólidas, a mesma observação pode ser feita para as distribuições pontilhadas (MUED). Corroborando o que discutimos, no início dessa seção, sobre distribuições adimensionais em decaimentos de cadeias curtas de partículas pesadas. As distribuições não são totalmente idênticas porque efeitos como PS, hadronização e cortes na geração dos eventos acabam atrapalhando a invariância com o espectro. Note que a distribuição $N_{\text {jet }}$ apresenta poucas diferenças também entre os dois modelos normalizados. Essa variável depende basicamente do matching de jatos, pois se esse processo não for realizado apropriadamente, temos uma influência direta na contagem de jatos após PS e hadronização. Em um cenário onde $m_{\tilde{\chi}_{1}}$ e $m_{B^{(1)}}$ são fixos, as diferenças são poucas quando não há cortes retangulares aplicados.

Ressaltamos que, neste trabalho, estamos desconsiderando qualquer correlação estatística entre observáveis. 

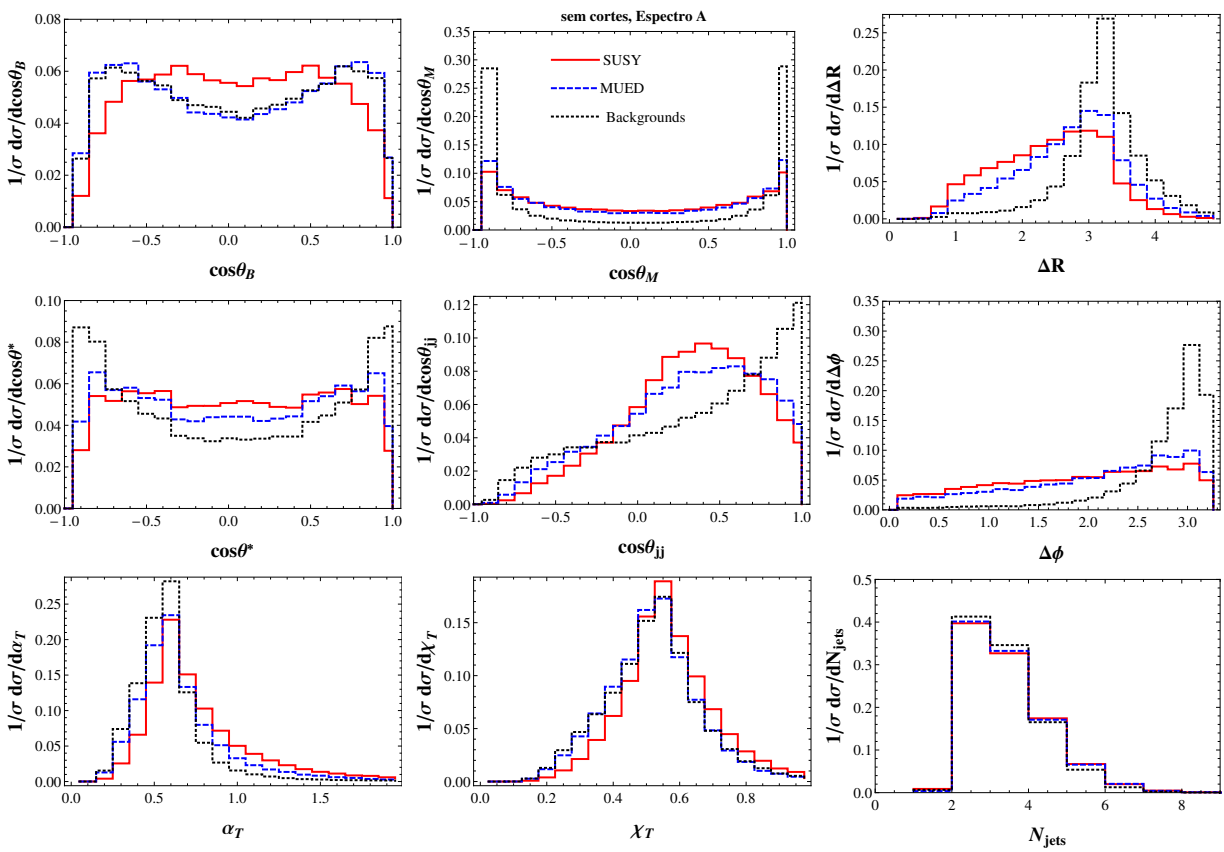

Figura 3.13: Diferenças nos formato das distribuições para os diferentes modelos. Pequenas diferenças nas formas da distribuições são levadas em conta em nossa análise multivariada. Após cortes retangulares esses formatos tendem a ficar parecidos, dificultando o discernimento de modelos. Por isso a necessidade de levar em conta uma estatística poderosa para a análise de descoberta e discernimento de modelos de nova física.

\subsection{Escaneamento de cortes retangulares}

Para os cortes escolhemos três variáveis para análise simultânea, $H_{T}, \mathscr{H}_{T}$ e $p_{T}$. Esses três observáveis são amplamente utilizados em análise de descoberta de nova física [91, 92, 93]. Escolhemos o espectro base, o mais leve, para aplicar a análise de cortes. Foi escolhido um intervalo de $600 \mathrm{GeV}$ a $2000 \mathrm{GeV}$ de passo $100 \mathrm{GeV}$ para $H_{T}$ e $\not_{T}$, e um valor fixo para o momento transverso dos dois jatos mais duros de cada evento de $400 \mathrm{GeV}\left(p_{T}\right)$. Além dessas variáveis também escolhemos um corte na pseudo-rapidez máxima dos dois jatos mais duros, $\eta_{\max }<2.5$. Estamos interessados em jatos mais centrais, pois estes serão os de origem mais promissora para descoberta de nova física. Fixamos também um espectro para esta análise, o Espectro B.

Para realizar o escaneamento integramos programas em PYTHON e CSHELL com o MADANALYSIS (versão 1.1.3). O MADANALYsis precisa ser configurado através do arquivo kin_func.f de modo a incluir as nove distribuições que descrevemos no início desse capítulo.

Realizamos o escaneamento em 225 conjunto de cortes diferentes, sendo que cada conjunto continha 6 canais de supersimetria e backgrounds e cada canal contem um conjunto de 40000 eventos, em média. O que totaliza por volta de 54 milhões de eventos analisados de sinal e backgrounds. Esse é um processo que demanda um tempo grande de análise. Ao término do escaneamento escolhe-se o corte que maximiza a significância de descoberta de supersimetria com a estatística de log-likelihood ratio.

\subsection{Correções NLO, Prospino}

O programa Prospino [94] baseado em Fortran possui rotinas para cálculos em NLO de processos para QCD supersimétrica. As correções de NLO para QCD supersimétrica aumentam as seções de choque de produção de pares de squarks, gluinos e de produção associada de squarkgluino em comparação com as seções de choque de LO, além de diminuírem a dependência de 

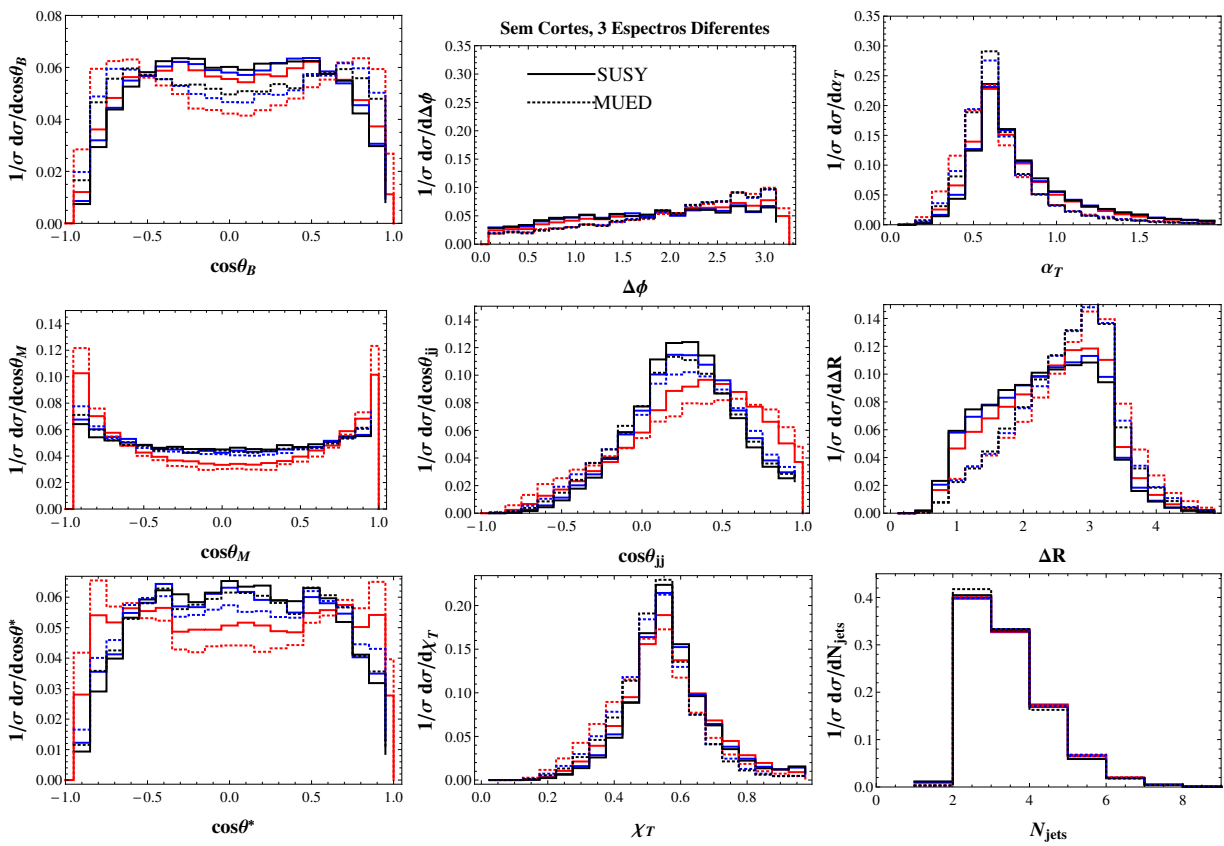

Figura 3.14: Diferenças no formato das distribuições para 3 dos 150 espectros estudados em nosso trabalho. Todas as curvas com $m_{\tilde{\chi}_{1}}=0.3 \mathrm{TeV}$ e $m_{\tilde{q}}=1.4 \mathrm{TeV}, m_{\tilde{g}}=1.5 \mathrm{TeV} ; m_{\tilde{q}}=2.6$ $\mathrm{TeV}, m_{\tilde{g}}=3.0 \mathrm{TeV} ; m_{\tilde{q}}=3.0 \mathrm{TeV}, m_{\tilde{g}}=4.0 \mathrm{TeV}$. Mesmo para diferentes massas, em cada uma dos respectivos modelos o formato da distribuição mantém-se aproximadamente o mesmo.

escalas de fatorização e renormalização. Correções eletrofracas também já foram calculadas para a produção de pares de squarks [95], mas não têm a mesma relevância quanto às de QCD e, portanto, não foram levadas em conta em nosso trabalho.

A determinação de incerteza na escala de fatorização, chamaremos de $\epsilon_{\text {fatoriz }}$, em alguns casos, não é um processo direto. É comum usar um processo de propagação de erros [71], devido à sua rápida implementação. Nesse método escolhe-se o valor da incerteza variando a escala de fatorização manualmente. A escala é variada em $2 \mu^{2}$ e $\frac{1}{2} \mu^{2}$, coleta-se a variação da seção de choque e compara-se com a seção de choque na escala de fatorização original $\mu^{2}$. A estimativa para o impacto dessa incerteza é:

$$
\epsilon_{\text {fatoriz }}=\frac{\sigma^{+}-\sigma^{-}}{\sigma^{0}} .
$$

Onde $\sigma^{ \pm}$indica a variação superior (inferior) da seção de choque com a escala, e $\sigma^{0}$, o valor da seção de choque na escala original de fatorização. A escala de fatorização $\left(\mu^{2}\right)$ é determinada de acordo com o que foi discutido na seção 3.6 .

Para os backgrounds, a correção NLO também é possível e aplicamos em nosso trabalho. Porém, infelizmente, o Prospino não calcula essas correções. Em todo caso é possível encontrar correções NLO para vários fenômenos do Modelo Padrão, veja [96, 97].

Fizemos um programa em PYTHON que integra-se ao Prospino, com isso foi possível obter 150 seções de choque para os 3 canais de supersimetria e avaliar o impacto da incerteza na escala de fatorização para essa seção de choque. Todos esses runs consomem um tempo razoável, apesar de pequeno quando comparado a outros procedimentos de nosso trabalho. Afinal, cada canal deve ser avaliado três vezes para um total de 150 espectros diferentes. Isso resulta em um total de 450 runs para encontrar seções de choque de produção e os erros sistemáticos devido à escala de fatorização. Quando isso é feito o programa prepara três runs seguidos alterando somente a escala de fatorização das PDF (particle data functions). Isso fornece três valores para um dado canal. Somando a contribuição dos 3 canais de supersimetria temos que a incerteza devido à 
escala de fatorização dada por 3.25 , torna-se:

$$
\epsilon_{4}=\frac{\left(\sigma_{\text {canal1 }}^{+}+\sigma_{\text {canal2 }}^{+}+\sigma_{\text {canal3 }}^{+}\right)-\left(\sigma_{\text {canal1 }}^{-}+\sigma_{\text {canal2 }}^{-}+\sigma_{\text {canal3 }}^{-}\right)}{\left(\sigma_{\text {canal1 }}^{0}+\sigma_{\text {canal2 }}^{0}+\sigma_{\text {canal3 }}^{0}\right)},
$$

onde, no caso de supersimetria para um dado espectro: canal1 $=\tilde{q} \tilde{q}$, canal2 $=\tilde{q} \tilde{q}^{*}$ e canal3 $=$ $\tilde{q} \tilde{g}$. Lembrando que as seções de choque de MUED estarão normalizadas pelas seções de choque de supersimetria, por isso levaremos em conta somente canais supersimétricos para a incerteza na escala de fatorização e o resultado se estende para MUED, para os backgrounds uma análise semelhante deve ser feita. Para os backgrounds, somente os canais de produção do bóson $Z$ e jatos são relevantes 3.5 .3 , com isso 3.25 torna-se:

$$
\epsilon_{3}=\frac{\left(\sigma_{\mathrm{zqq}}^{+}+\sigma_{\mathrm{zqg}}^{+}+\sigma_{\mathrm{zgg}}^{+}\right)-\left(\sigma_{\mathrm{zqq}}^{-}+\sigma_{\mathrm{zqg}}^{-}+\sigma_{\mathrm{zgg}}^{-}\right)}{\left(\sigma_{\mathrm{zqq}}^{0}+\sigma_{\mathrm{zqg}}^{0}+\sigma_{\mathrm{zgg}}^{0}\right)} .
$$

Em nosso caso, para o LHC $14 \mathrm{TeV}, \epsilon_{3}=5 \%$.

Na tabela 3.4 mostramos somente alguns valores da incerteza sistemática na taxa dos eventos de supersimetria devido à escala de fatorização. Note que, embora estejamos usando uma correção em NLO, a escala de fatorização introduz uma incerteza grande quando variada. Isso porque a convergência das funções de densidade partônica não é boa para altas energias, vide [98].

\begin{tabular}{|c|c|c|}
\hline$m_{\tilde{q}}(\mathrm{TeV})$ & $m_{\tilde{g}}(\mathrm{TeV})$ & $\epsilon_{4}(\%)$ \\
\hline 1.4 & 1.5 & 10.3 \\
2.2 & 2.0 & 12.7 \\
3.0 & 4.0 & 13.3 \\
5.0 & 5.0 & 22.1 \\
\hline
\end{tabular}

Tabela 3.4: Erro sistemático na seção de choque devido à variação da escala de fatorização em NLO para supersimetria. Tabela parcial de resultados.

\subsection{Identificação de jatos de quarks e glúons (Tagging)}

Squarks e gluinos decaem invariavelmente em jatos de quarks. Por outro lado, o background dominante para jatos + MET, $Z+$ jatos, possui radiação de QCD proveniente da emissão de quarks e glúons. Se for possível identificar os jatos de quarks e glúons, podemos vetar os identificados com a emissão de glúons e assim suprimir ainda mais os eventos de MP, ainda que a emissão de glúons também ocorra nos eventos de sinal. Mostraremos que o esforço de identificar jatos de quarks e glúons (tagging) é, de fato, vantajoso para o objetivo de aumentar a significância do sinal.

A identificação de jatos é realizada através de análises da estrutura interna de jatos. Quando possível, algumas informações extras podem ser usadas como o branching ratio de um determinado processo. Conhecendo a topologia exata envolvida no processo é possível identificar a origem de um dado jato, infelizmente quando o número de processos envolvidos é grande tornase complicado determinar qual topologia foi usada no processo de produção de um determinado jato. Em geral a produção de jatos de glúons é favorecida em processos de QCD, pois os fatores de cor (Casimir) para quarks e glúons diferem consideravelmente, $\frac{C_{A}}{C_{F}}=2.25$. Isso leva a uma produção de jatos de glúons em média 2 vezes maior que a produção de jatos de quarks. Além do que, jatos de sabores pesados tendem a ser semelhantes aos jatos de glúons, ou seja, jatos menos colimados. Todas essas informações juntamente com a estrutura dos jatos podem indicar se um quark leve ou glúons os produziu. 
A análise conduzida em [21] propõe uma técnica para a realização do tagging de jatos de quarks e glúons. Nesse trabalho mostrou-se que observáveis como o número de traços reconstruídos dentro de um jato e uma espécie de largura (distância) entre esses traços podem ser usados para a realização do tagging. Jatos duros e centrais $|\eta|<3$ são os que possuem melhores resultados para o tagging de jatos.
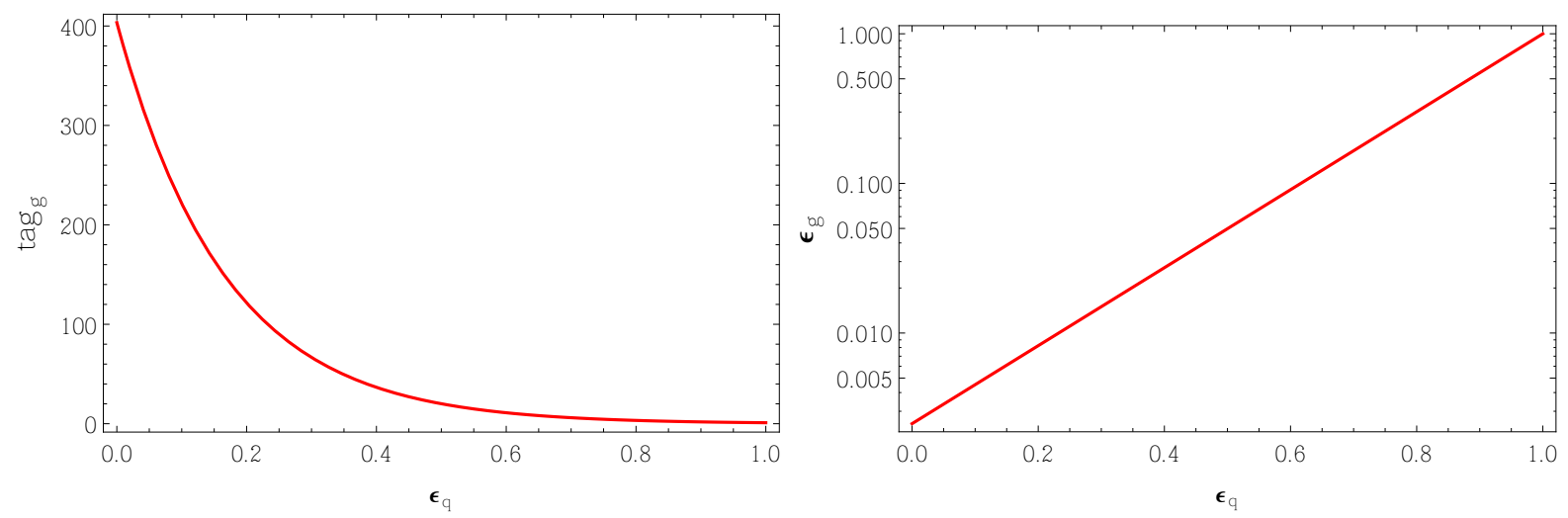

Figura 3.15: Curvas para o tagging de jatos de quarks e glúons, uma para $\operatorname{tag}_{g}$ e outra para o recíproco $\epsilon_{g}$, a primeira retirada de [21]. Essa curva tem maior validade para jatos de $p_{T}>200$ $\mathrm{GeV}$ e centrais.

É possível inferir a função que relaciona a aceitação de quarks $\epsilon_{q}$ com a exclusão de glúons $\operatorname{tag}_{g}$

$$
\operatorname{tag}_{g}=e^{6\left(1-\epsilon_{q}\right)}
$$

onde $\epsilon_{g}=\frac{1}{\operatorname{tag}_{g}}$, veja Fig 3.15. Essa relação foi obtida através de resultados de [21] e a região de maior eficiência para esse tagging é com jatos de $p_{T}>200 \mathrm{GeV}$.

Em nosso trabalho usamos o tagging como um fator global na taxa de produção de jatos e MET. Como simulamos nossos eventos em uma análise onde cada canal é conhecido completamente, e sabemos qual jato teve sua origem em quarks ou glúons, podemos aplicar esse fator de diluição na seção de choque de produção de cada canal. Por exemplo, se o fator de seleção de jatos de quarks for de $\epsilon_{q}=60 \%$ então teremos que somente $\epsilon_{g}=\frac{1}{11}$ dos glúons passarão pelo tagging de jatos (3.28). Podemos esperar que canais que contribuem com 2 jatos de quarks terão uma fator de diluição na seção de choque total de produção de jatos vindos de quarks de $36 \%$ e um fator de diluição na seção de choque de produção para glúons de $1 \%$.

O ATLAS já faz uso da técnica de tagging de jatos de quarks e glúons em suas análises para busca de nova física [22].

\subsection{Distribuições finais, aplicação de tagging, K-factors e nor- malização}

Nesta seção dedicaremos espaço para explicitar como contabilizamos as: contribuições de sinal e backgrounds, aplicação dos fatores de tagging de quarks e glúons, correções em NLO, eficiências de cortes retangulares e normalização para seções de choque de MUED.

A hierarquia de massa é relevante aqui. Para a região $m_{\tilde{g}}>m_{\tilde{q}}$ temos três canais contribuindo para o nosso sinal. Já na região $m_{\tilde{g}}<m_{\tilde{q}}$ os canais com gluínos deixam de contribuir para um estado final do tipo jatos + MET, e portanto eles devem ser excluídos da análise nessa região.

Para realizar todas essas tarefas criamos um programa ForTRAn. As entradas desse programa são todos os bins das nove distribuições escolhidas para a análise. 
- $\sigma_{\text {lhco }}^{i}$ a seção de choque dada pelo MAdGRAPH5 logo após a geração dos eventos para o canal $i$.

- $\sigma_{\text {cut }}^{i}$, a seçaõ de choque dada pelo MADANALYsis logo após aplicação de cortes para o canal $i$.

- $\sigma_{\mathrm{NLO}}^{i}$, seção de choque em NLO para o canal $i$ supersimétrico.

- $\epsilon_{q}$, tagging de jatos de quarks. Trata-se de um parâmetro livre, valores possíveis estão entre 0 e 1.0 .

- $\epsilon_{g}=\frac{1}{\operatorname{tag}_{g}}$, tagging de jatos de glúons 3.28.

Para ilustração vamos analisar o canal de produção de squarks, denominaremos sqsq. O bin $I$ da distribuição $J$ fica alterado da seguinte maneira:

$$
s q s q_{\text {new }}(J, I)=s q s q_{\mathrm{old}}(J, I) \cdot \frac{\sigma_{\mathrm{NLO}}^{s q s q}}{\sigma_{\text {lhco }}^{s q s q}} \cdot \epsilon_{q}^{2} .
$$

O mesmo procedimento é feito para os canais squark/anti-squark e squark/gluino (quando a hierarquia de massas permitir). O fator de tagging é o mesmo para todos os canais que contribuem para o sinal, pois em todos os canais os jatos mais duros são de quarks provenientes do decaimento direto de partículas supersimétricas (ou de dimensões extras) pesadas. Lembrando que nossos histogramas estão normalizados pela respectiva seção de choque então a normalização de $s q s q_{\text {new }}$ para um dado histograma é $\sigma_{\text {cut }}^{s q s q} \cdot \frac{\sigma_{N \text { NLO }}^{s q s q}}{\sigma_{\text {lhco }}^{\text {squ }}}$. O que denota que para uma distribuição sem cortes a correção NLO é $\sigma_{\mathrm{NLO}}^{\text {sqsq }}$, em consequência, um histograma que passou por cortes retangulares tem a correção NLO fracionada $\rrbracket^{4}$ Assim não estaremos superestimando o sinal.

Para os eventos de MUED, como ilustração usaremos o canal de produção de KK-quarks, denominaremos $q 1 q 1$. A normalização desse canal é,

$$
q 1 q 1_{\text {new }}(1, I)=q 1 q 1_{\text {old }}(1, I) \cdot \frac{\sum_{I} s q s q_{\mathrm{new}}(1, I)}{\sum_{I} q 1 q 1_{\mathrm{old}}(1, I)} .
$$

Note que fizemos como ilustração uma normalização da distribuição cinemática $1(J=1)$ para o canal de produção de KK-quarks, mas isso pode ser feito sobre qualquer distribuição. Esse processo garante que todos os eventos de MUED terão a mesma seção de choque. Observe que $s q s q_{\text {new }}$ já possui um fator de tagging na seção de choque, garantindo que MUED também já tenha um tagging de jatos de quarks aplicado.

Depois de somado todas as contribuições de cada canal, levando em conta a hierarquia de massa para cada espectro, temos a contribuição de supersimetria e dimensões extras universais definidas.

Para os backgrounds os K-factors de produção de $\mathrm{Z}+$ jatos é $K_{\mathrm{Z}}+$ jets $=1.10$ [96, 97]. A diferença agora é que deveremos aplicar fatores de tagging de jatos de forma diferente, pois nossos backgrounds têm jatos vindos de glúons.

- Para o canal $Z+q q$, onde $q$ é um jato de quark o fator de tagging é: $\epsilon_{q}^{2}$.

- Para o canal $Z+q g$, onde $q$ é um jato de quark e $g$ um jato de glúon o fator de tagging é: $\epsilon_{q} \cdot \epsilon_{g}$.

- Para o canal $Z+g g$, o fator de tagging é: $\epsilon_{g}^{2}$.

Note que, tanto sinal e backgrounds, levam contribuições diferentes de fator de tagging. Isso garante um expressivo aumento no poder de discernimento.

\footnotetext{
${ }^{4} \mathrm{~A}$ correção NLO para a seção de choque não considera cortes de qualquer natureza. A seção de choque calculada após a geração de nossos eventos possui cortes, porém são cortes pequenos.
} 


\subsection{Incertezas sistemáticas no log-likelihood ratio}

Todas as análises do nosso trabalho levam em conta erros sistemáticos. A escolha de cortes, melhor tag de jatos, a estatística-teste, todos serão analisados com erros sistemáticos na taxa e formato dos eventos.

Retomando a equação do likelihood que apresentamos em (2.34), e os resultados discutidos na seção 2.9, temos que a aplicação de incertezas nas taxas dos eventos pode ser realizada através da marginalização do likelihood original,

$$
\mathcal{L}=\prod_{i=1}^{N} \mathcal{P}\left(n_{i} \mid \mu_{i}\right) \mathcal{G}\left(L \mid \tilde{L}, \sigma_{L}\right) .
$$

A função $\mathcal{G}\left(L \mid \tilde{L}, \sigma_{L}\right)$ é uma gaussiana normalizada a unidade, de média $\tilde{L}$ e desvio padrão $\sigma_{L}$. Em nosso caso, analisamos cenários de $\tilde{L}=100,500$ e $3000 \mathrm{fb}^{-1}$ e $\sigma_{L}$ assumirá valores correspondentes a diferentes tipos de erros sistemáticos. O único parâmetro livre para a marginalização é $\sigma_{L}$, cada tipo de incerteza sistemática terá associada um desvio padrão gaussiana apropriado. Denotaremos esses desvios por $\epsilon_{i}$.

A determinação dos $\epsilon_{i}$, em alguns casos, não é um processo direto. Por exemplo, no caso da incerteza na escala de fatorização, vimos na seção 3.10, que variações da escala fornecem uma estimativa desses erros. Em outros casos, medidas auxiliares podem ser usadas para estimar os erros em um determinado aparato, como as medidas realizadas pelo ATLAS para análise da incerteza sistemática na luminosidade integrada [99].

Aplicamos quatro tipos de incertezas sistemáticas nas taxas dos nossos eventos. São,

- Incerteza na escala de fatorização para os eventos de background. Em nosso caso esse valor foi calculado explicitamente, $\epsilon_{3}=5 \%$.

- Incerteza na escala de fatorização do sinal. Já apresentamos essa incerteza na Tab. 3.4 A terceira coluna da tabela será o valor dessa incerteza, definimos como $\epsilon_{4}$. O valor dessa incerteza varia de $10 \%$ para espectros leves a $22 \%$ para espectros pesados, vide seção.

- Incerteza na luminosidade integrada $\left(\epsilon_{5}\right)$. Baseados em resultados da colaboração ATLAS, o valor dessa incerteza está definida hoje em $3.5 \%$.

- Uma incerteza sistemática que pode estar relacionada a erros sistemáticos de diversas fontes. Como por exemplo, convergência da série infinita de QCD, eficiência de geradores de Monte Carlo, eficiência de tagging de jatos de quarks e glúons, eficiência do programa Pүтнia no parton shower e na hadronização, dentre outras [66]. Chamaremos essa incerteza de Variadas, será dada por $\epsilon_{\text {taxa }}$. O valor associado à essa incerteza é variado livremente, escolhemos cenários de 0,10 e $20 \%$. Procuras de supersimetria estimam que essas incertezas variam de 20 a $100 \%$ no LHC [100], é claro que esses valores dependem do controle dos erros sistemáticos, com o passar do tempo as incertezas sistemáticas tendem a diminuir conforme o conhecimento sobre o aparato experimental melhora.

Com isso formamos o conjunto de incertezas sistemáticas nas taxas dos eventos de nossa análise, denotadas pelo conjunto $\left\{\epsilon_{\text {taxa }}, \epsilon_{3}, \epsilon_{4}, \epsilon_{5}\right\}$.

Nossa aplicação de incertezas sistemáticas seguiu o critério de que somente bins ocupados foram usados na análise. Eventualmente bins com ocupação zero tanto para os backgrounds quanto para o sinal foram excluídos do cálculo da significância do teste estatístico.

As incertezas no formato das figuras têm implementações diferentes das incertezas nas taxas, elas podem ter origens na incerteza da escala de energia para os jatos, esse efeito influencia todos os jatos de um dado evento na mesma direção. Ou pode ter origem em incertezas estatísticas nas amostras de Monte Carlo, esse tipo de incerteza é interessante em nosso trabalho, pois na prática, as distribuições de nossa análise têm estatística limitada, afinal foram geradas por um 
número finito de eventos através de geradores de Monte Carlo. É possível levar esse tipo de limitação como uma incerteza sistemática [71]. A prescrição é verificar a condição,

$$
N_{\mathrm{MC}}<10 N_{\text {dados }} .
$$

$N_{\mathrm{MC}}$ é o número de eventos de MC que passaram nos cortes, esse número é dado pelo MADANALYSIS. Depende, em nosso caso, da eficiência de matching de jatos e principalmente de cortes retangulares, como discutidos nas sub-seção 3.6.1 e seção 3.7. E $N_{\text {dados }}$ é o número de eventos dado pela seção de choque multiplicada pela luminosidade integrada, incluindo eficiência de corte, tagging de jatos e correções NLO. Quando a condição 3.32 é verdadeira, estamos estimando, relativamente, muitos eventos de dados de uma amostra de MC insuficiente para essa afirmação, isso denota um problema. A prescrição para contornar essa limitação é inserir um erro sistemático bin a bin em cada distribuição. O número de eventos esperados em cada bin será flutuado de acordo com uma distribuição de Poisson, isso é uma espécie de erro sistemático no formato das distribuições. Esse erro é tratado através de marginalização, assim como as incertezas sistemáticas na taxa dos eventos.

Fizemos a avaliação espectro a espectro dos pontos que necessitavam desse tipo de incerteza. Para o sinal nossos $N_{\mathrm{MC}}$ são números grandes quando comparados com o background, basicamente porque os eventos de sinal passam mais nos cortes. E ainda, o aumento das massas de squarks e gluinos contribui para que mais eventos passem pelos cortes. Já as seções de choque, diminuem gradativamente enquanto o espectro aumenta, fazendo com que a condição (3.32) seja verdadeira em apenas $10 \%$ dos casos para uma análise sem tagging de jatos. Esse número cai para menos de $5 \%$ para uma análise com tagging de $\epsilon_{q}=0.5$. Já para o background temos o comportamento oposto, $N_{\mathrm{MC}}$ não passa de 500 eventos. Porém a seção de choque é sempre alta, pois são processos da QCD pura. Em todos os cenários possíveis de tagging $\left(0.1<\epsilon_{q}<1.0\right)$, com correção NLO, por exemplo, a relação 3.32 é satisfeita, portanto, é primordial incluir esse efeito no background e secundário no sinal.

O likelihood (2.34), com a inserção de incerteza sistemática na forma das distribuições, fica alterado da seguinte maneira,

$$
\mathcal{L}=\prod_{i=1}^{N} \mathcal{P}\left(n_{i} \mid \mu_{i}\right) \mathcal{P}\left(\tilde{b}_{i} \mid b_{i}\right) .
$$

Ressaltamos que retiramos da análise bins que não possuem eventos, tanto de sinal quanto de backgrounds.

Na Fig. 3.16 temos uma ilustração do que pode acontecer em um bin da distribuição $\chi_{T}$ de nossa análise levando em conta a incerteza no formato. Veja que a distribuição $\chi_{T}$, para os backgrounds, após cortes retangulares, tem seu formato prejudicado. A aplicação de uma flutuação em cada bin, de acordo com uma Poisson (setas vermelhas), é um método rápido para levar em conta o efeito de baixa estatística após cortes duros. Na tabela 3.5 temos uma visão geral das incertezas que incluiremos nesse trabalho.

\begin{tabular}{lccl}
\hline \hline Fonte da Incerteza & taxa (\%) & formato & Processos Afetados \\
\hline PDF & 5 & - & background \\
PDF & $10-22$ & - & sinal \\
Luminosidade & 3.5 & - & sinal e background \\
Variadas & $0-20$ & - & background \\
Número finito eventos MC & - & $\checkmark$ & background \\
\hline
\end{tabular}

Tabela 3.5: Natureza e módulo das incertezas sistemáticas aplicadas neste trabalho.

O trabalho computacional necessário para inserção de incertezas sistemáticas no likelihood construído através de histogramas, refere-se ao sorteio de cinco gaussianas diferentes, sorteios de 


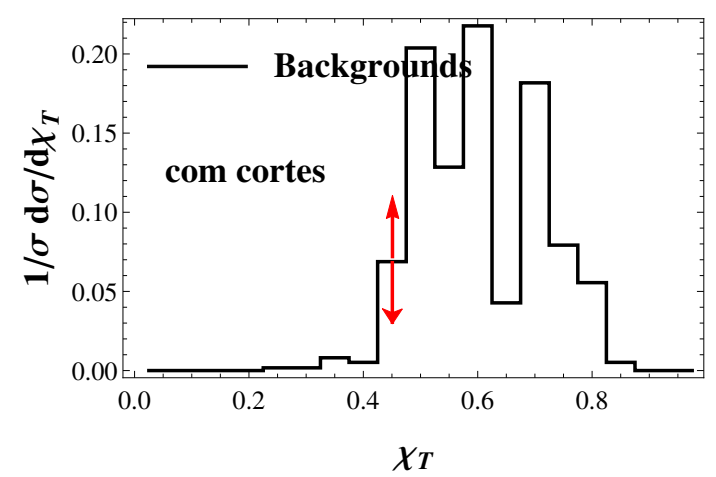

Figura 3.16: Ilustração do possível efeito de um erro sistemático no formato da distribuição cinemática $\cos \theta_{B}$.

Poisson bin-a-bin, da concomitante realização dos pseudo-experimentos e construção das PDF's conjuntas para a estatística-teste. Em média há um aumento de tempo de cálculos, para o nosso poder de processamento, da ordem de $50 \%$ quando são inseridos os erros sistemáticos. O processo de construção das PDFs, para a hipótese nula e alternativa, para todos os espectros, para todos os cenários analisados e para descoberta e discernimento de nova física ficou em torno de noventa horas. Esse é um tempo de processamento que consideramos médio para nossa capacidade computacional, em comparação com outros processos de nosso trabalho.

A aplicação do erro sistemático impacta principalmente no desvio padrão das PDFs das hipóteses nula e alternativa. O aumento dos erros sistemáticos faz com que as distribuições se alarguem, enquanto que seus valores médios permanecem praticamente intactos, como podemos ver na Fig. 3.17. Isso ocorre porque as incertezas são inseridas como sorteios gaussianos de média zero e desvio padrão 1 (3.31), para cada pseudo-experimento. Veremos no capítulo Resultados, que a incerteza sistemática limita completamente os nossos resultados. Com certeza, a implementação desses efeitos é trabalho obrigatório para análises que pretendem explicar a manifestação de dados experimentais além do Modelo Padrão no LHC.

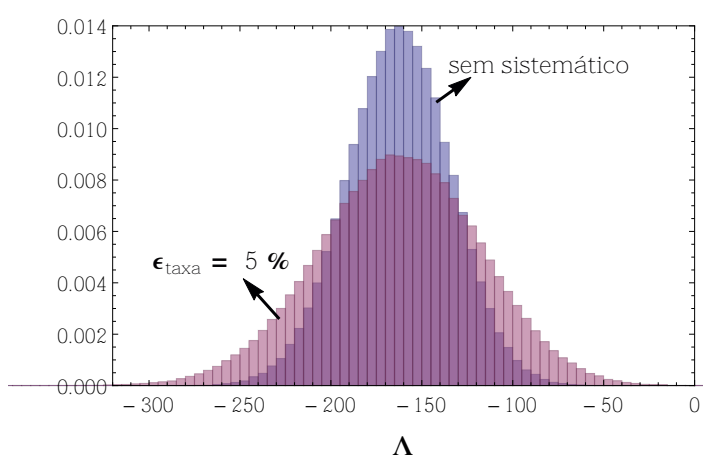

Figura 3.17: Manifestação de um erro sistemático na taxa dos eventos dos backgrounds na PDF da estatística-teste $\Lambda$, para um dado teste de hipóteses. O alargamento das gaussianas é decorrente da inserção de incerteza sistemática na taxa dos eventos.

O conjunto de incertezas sistemáticas usados em nosso trabalho é mínimo quando comparado ao que é feito pelas colaborações do CERN, embora seja um conjunto expressivo quando comparado com outros trabalhos de fenomenologia.

Antes de inciarmos o capítulo Resultados, iremos expor, na próxima seção, a terminologia que usaremos como forma de sintetizar a exposição dos resultados de nossa análise. 


\subsection{Cenários}

Consideramos 150 espectros diferentes, porém a exposição de alguns resultados parciais e verificações devem ser feita em pontos específicos do espaço de massas. Para isso definiremos os seguintes espectros:

- Espectro A: $m_{\tilde{q}}=m_{q^{(1)}}=1.4 \mathrm{TeV}, m_{\tilde{g}}=m_{g^{(1)}}=1.5 \mathrm{TeV}$ e $m_{\tilde{\chi}_{1}}=m_{B^{(1)}}=0.3 \mathrm{TeV}$.

- Espectro B: $m_{\tilde{q}}=m_{q^{(1)}}=2.6 \mathrm{TeV}, m_{\tilde{g}}=m_{g^{(1)}}=4.0 \mathrm{TeV}$ e $m_{\tilde{\chi}_{1}}=m_{B^{(1)}}=0.3 \mathrm{TeV}$.

- Espectro C: $m_{\tilde{q}}=m_{q^{(1)}}=1.4 \mathrm{TeV}, m_{\tilde{g}}=m_{g^{(1)}}=1.5 \mathrm{TeV}$ e $m_{\tilde{\chi}_{1}}=m_{B^{(1)}}=1 \mathrm{TeV}$.

Para as incertezas sistemáticas teremos os seguintes cenários:

- Sem Sistemáticos, aqui não temos a inserção de nenhum tipo de incerteza sistemática. Ainda haverá casos em que denotaremos $\epsilon_{\text {taxa }}=0 \%$, e novamente, todos os erros sistemáticos na taxa e formato serão desconsiderados.

- Sistemático no Background, nesse caso somente incertezas sistemáticas que afetam exclusivamente as taxas dos eventos de background são consideradas. Tratam-se de incertezas sistemáticas devido à escala de fatorização, luminosidade e à fontes diversas moduladas por $\epsilon_{\text {taxa }}$, vide seção 3.13 .

- Todos Sistemáticos, inclusão de todas incertezas sistemáticas listadas na tabela [3.5.

Para os resultados, a incerteza sistemática no formato das distribuições foi levada em conta somente quando $\epsilon_{\text {taxa }}>0$.

Para os cortes retangulares também é possível alternarmos em dois cenários:

- Sem Cortes, trata-se do caso onde não aplicam-se cortes em $H_{T}, \mathbb{H}_{T}$ e $p_{T}$.

- Cortes Ótimos, refere-se aos cortes que maximizam a descoberta de supersimetria para luminosidade integrada $(L)$ de $100 \mathrm{fb}^{-1}$, sem tagging de jatos aplicados, com inserção de todas as incertezas sistemáticas consideradas na análise (Todos Sistemáticos) e para o Espectro B. Trata-se do conjunto $H_{T}=1500 \mathrm{GeV}, \mathbb{H}_{T}=1250$ e $p_{T}=400 \mathrm{GeV}$. Note que toda a análise para os cortes foi realizada para um espectro específico.

O cenário de Cortes Ótimos foi analisado somente para $L=100 \mathrm{fb}^{-1}$, esse é um cenário de menor luminosidade em nosso trabalho, portanto, será o cenário de maior dificuldade para descoberta de supersimetria.

O tagging de jatos de quarks e glúons, que maximiza a descoberta de supersimetria, depende da luminosidade integrada e eficiências. Por isso valores específicos de luminosidade terão um tagging fixo $\left(\epsilon_{q}\right)$. Veremos mais adiante como determinamos esses valores. Por enquanto, iremos nos ater a definir os possíveis cenários para o tagging de jatos de quarks e glúons:

- $L=100 \mathrm{fb}^{-1}$, nesse caso $\epsilon_{q}=0.53$ e $\epsilon_{g}=\frac{1}{11}$.

- $L=500 \mathrm{fb}^{-1}$, nesse caso $\epsilon_{q}=0.38$ e $\epsilon_{g}=\frac{1}{41}$.

- $L=3000 \mathrm{fb}^{-1}$, nesse caso $\epsilon_{q}=0.32$ e $\epsilon_{g}=\frac{1}{59}$.

Eventualmente, teremos casos onde desconsideramos o tagging de jatos, chamaremos de sem tagging. Nesse caso $\epsilon_{q}=\epsilon_{g}=1$. 


\subsection{A escolha de cortes retangulares}

Os cortes retangulares foram definidos de modo a maximizar a descoberta de supersimetria para o teste estatístico $Z_{L L R}$, para um cenário onde $L=100 \mathrm{fb}^{-1}$, o conjunto Todos Sistemáticos $\operatorname{com} \epsilon_{\text {taxa }}=20 \%$, Espectro B, sem a inclusão de tagging de jatos de quarks e glúons e com nove observáveis físicos. Com exceção do espectro, o cenário listado é o pior para descoberta de supersimetria, por isso o definimos como referência para o escaneamento de cortes.

Como estamos avaliando três variáveis diferentes para os cortes, que possuem um grande potencial de separação de sinal e backgrounds, veja Fig. 3.10a a 3.10c, decidimos selecionar duas delas, onde a sensibilidade de $Z_{L L R}$ é maior, e então aplicar um escaneamento bi-dimensional. Notamos que $Z_{L L R}$ era menos sensível à variável $p_{T}$. Então escolhemos um valor para fixar essa variável, obtivemos bons valores para $Z_{L L R}$ em um escaneamento com $p_{T}=400 \mathrm{GeV}$, e então fizemos um escaneamento em $H_{T}$ e $\mathscr{H}_{T}$. Na Fig. 3.18 temos o resultado desse escaneamento. Podemos perceber que quanto mais duro for o corte em $\mathbb{F}_{T}$, temos significâncias cada vez maiores. Porém regiões com $\mathbb{E}_{T}>1600 \mathrm{GeV}$ oferecem um número pequeno de eventos de sinal para essa luminosidade e espectro. Escolhemos um conjunto de cortes onde $Z_{L L R}$ fosse relativamente alta, e o número de eventos de sinal estivessem na ordem de 1000 para $L=100 \mathrm{fb}^{-1}$. Essa premissa é necessária pois estamos analisando um cenário onde não há tagging de jatos. Após a aplicação do tagging, esse número de eventos irá diminuir consideravelmente.

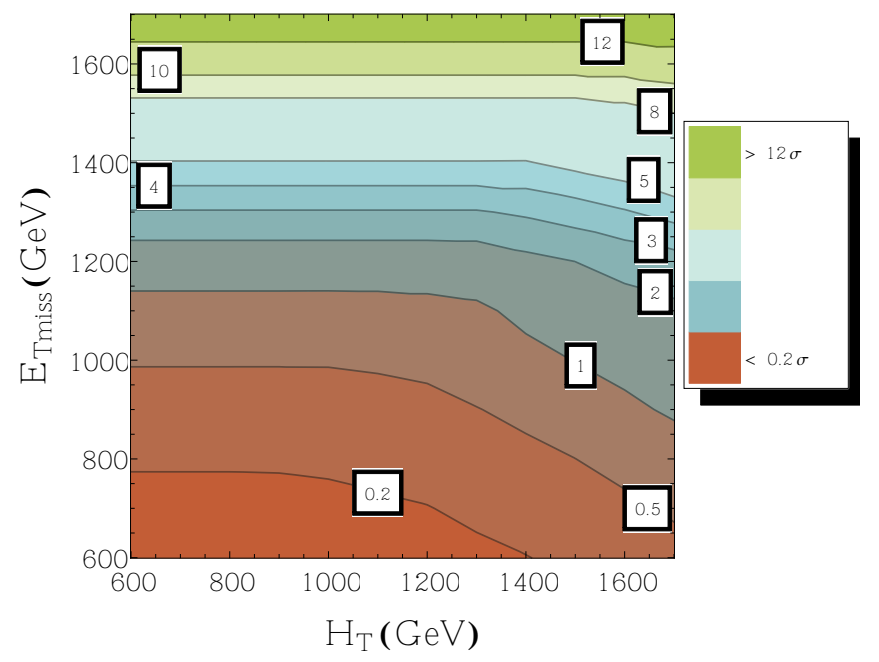

Figura 3.18: Escaneamento de cortes retangulares para descoberta de supersimetria. Os números destacados indicam curvas de nível para $Z_{L L R}$.

Escolhemos o conjunto de cortes de $H_{T}=1500 \mathrm{GeV}, \not_{T}=1250 \mathrm{GeV}$ e $p_{T}=400 \mathrm{GeV}$. Definimos esse conjunto como o Cortes Ótimos. Para esses cortes, temos as seguintes seções de choque total para os espectros descritos na seção 3.14 .

- Espectro A, $\sigma^{\text {susy }}=8.80 \mathrm{fb}$ e $\sigma^{\text {bckg }}=0.88 \mathrm{fb}$.

- Espectro B, $\sigma^{\text {susy }}=0.34 \mathrm{fb}$ e $\sigma^{\text {bckg }}=0.88 \mathrm{fb}$.

- Espectro C, $\sigma^{\text {susy }}=0.26 \mathrm{fb}$ e $\sigma^{\text {bckg }}=0.88 \mathrm{fb}$.

\subsection{6 $Z_{s b}$ e $Z_{L L R}$, revisitado}

Definidos os cenários de nosso trabalho, podemos analisar a significância em um contexto direcionado à nossa análise. Como vimos em $(2.42), Z_{s b}$ leva em conta somente uma informação, o número de eventos de sinal e de background. Em compensação, $Z_{L L R}$ é sensível à forma das 
distribuições e ainda é possível utilizar diversos observáveis físicos diferentes 2.6. A vantagem de incluir diversas distribuições diferentes pode ser vista na Fig. 3.19. Note que a inserção de nove distribuições leva $Z_{L L R}$ a quase triplicar o seu valor comparado à uma análise com apenas uma distribuição, enquanto que a curva para $Z_{s b}$ não tem sensibilidade para inserção de diferentes distribuições. Para $N_{\text {distri }}=1$ temos que a análise multivariada possui métrica maior que a contagem de eventos, demonstrando mais uma vez o poder da estatística-teste razão log-likelihood. A Fig. 3.19 foi gerada a partir do Espectro B e luminosidade integrada de 100 $f b^{-1}$, não usamos tagging de jatos de quarks e glúons.

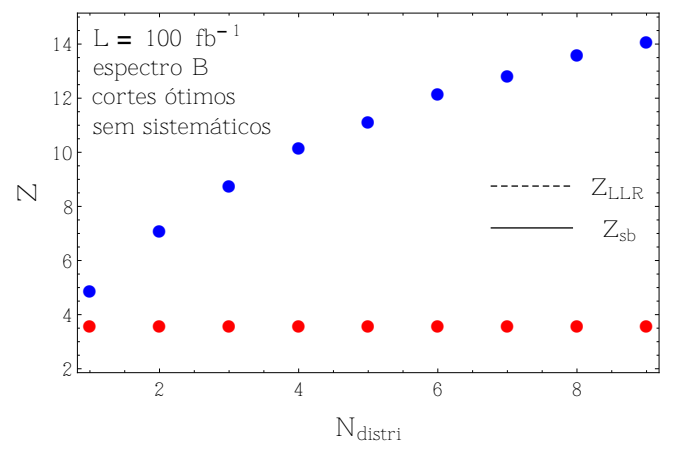

Figura 3.19: Comparação entre os testes $Z_{s b}$ e $Z_{L L R}$ com relação ao número de observáveis usados na análise multivariada.

Verificamos que o comportamento de $Z_{L L R}$ com o número de histogramas inseridos é semelhante ao observado na Fig. 3.19 em todos os espectros da análise. Esse comportamento é esperado, pois as equações 2.37 e 2.39 beneficiam-se quando aumentamos $N_{\text {distri }}$. Em geral, essas equações tornam-se mais positivas ou negativas dependendo da razão $s_{i j} / b_{i j}$, e isso acaba mudando o valor médio da estatística-teste. Ressaltamos que a aplicação de incertezas sistemáticas alteram o padrão observado para $Z_{L L R}$ na Fig. 3.19, porque se a distribuição cinemática não possuir relativa diferença de formato entre sinal e background, qualquer flutuação estatística do background pode desaparecer com o sinal.

Como já vimos anteriormente na seção 2.8, para a contagem de eventos, dependendo dos erros sistemáticos e da razão $\frac{s}{b}$, em alguns casos é impossível alcançar descoberta ou $5 \sigma$ de significância. Na Fig. 3.20, vemos que esse comportamento não é exclusividade de $Z_{s b}$, pois $Z_{L L R}$ também tem essa característica, embora ele seja alcançado em regiões com um valor de significância maior. Perceba também que o teste $Z_{s b}$, sem a aplicação de incertezas sistemáticas, não consegue minimamente reproduzir o comportamento real de qualquer análise que leve efeitos de incertezas. Aqui, $Z_{L L R}$ é calculado usando os nove observáveis físicos definidos na seção 3.8. Conforme a incerteza sistemática na taxa dos eventos de background aumenta, a saturação da significância ocorre com valores menores de luminosidade integrada. Essa saturação ocorre em luminosidades integradas aproximadamente iguais para a contagem de eventos e razão loglikelihood. No cenário ilustrado, com $\epsilon_{\text {taxa }}=5 \%$ a saturação ocorre por volta de $2 a b^{-1}$, para $\epsilon_{\text {taxa }}=10 \%$ por volta de $1.5 a b^{-1}$ e finalmente para $\epsilon_{\text {taxa }}=20 \%$ ocorre em $500 \mathrm{fb}^{-1}$ Fig. 3.20 .

Realizamos outra verificação, para erros sistemáticos, como pode ser visto na Fig. 3.21, para descoberta de supersimetria. Das três curvas esboçadas, duas delas representam $Z_{s b}$, para um caso onde não há incerteza sistemática 2.40) e outra com uma incerteza sistemática na taxa dos eventos de backgrounds, de acordo com o conjunto Sistemático no Background 2.42). A terceira curva representa a métrica de significância, $Z_{L L R}$, com a análise de apenas um histograma $2^{5}$ como observável físico para descoberta de supersimetria. Inserimos somente uma distribuição pois assim podemos comparar $Z_{L L R} \operatorname{com} Z_{s b}$ em bases equivalentes. Porque, em geral, utilizar um teste estatístico com nove observáveis físicos diferentes trará melhores resultados quando comparado a um teste que utiliza somente um.

\footnotetext{
${ }^{5} \mathrm{O}$ observável físico usado em todas as nossas análises com apenas um histograma foi $\cos \theta_{M} 3.8$
} 


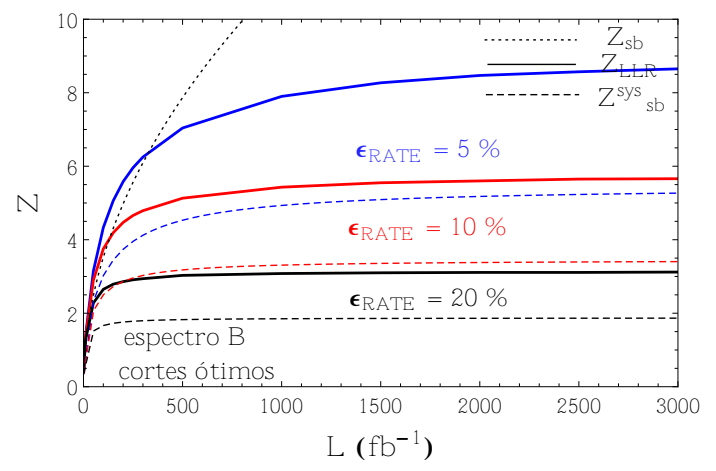

Figura 3.20: Comparação entre $Z_{L L R}$ e $Z_{s b}$, em diferentes cenários para $\epsilon_{\text {taxa }}$.

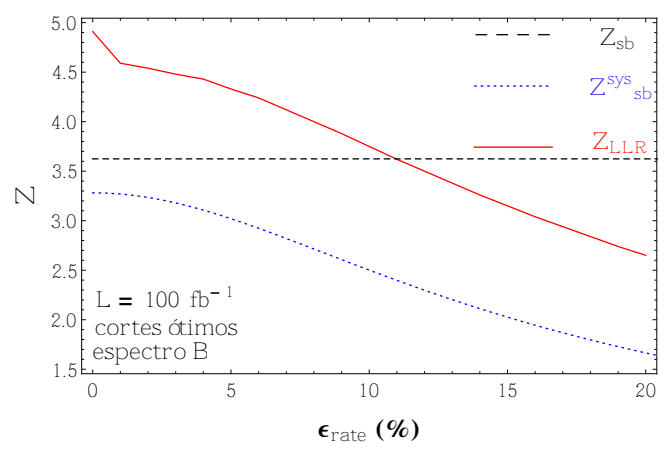

Figura 3.21: Comparação entre os testes estatísticos $Z_{L L R}$ e $Z_{s b}$ com relação à influência de uma incerteza sistemática na taxa dos eventos de background.

Vemos na Fig. 3.21 que, em nosso caso, $Z_{L L R}$ é um teste estatístico com maior poder de descoberta em qualquer cenário com inserção de incertezas sistemáticas, quando comparado com o $Z_{s b}$. Mesmo nos cenários onde usamos apenas um observável para descoberta de nova física. Isso deve-se ao fato de que, para obter $Z_{L L R}$, usamos as mínimas diferenças no formato da distribuição para o sinal e background, em uma análise bin a bin. Enquanto que $Z_{s b}$ leva em conta, somente, o número de eventos total.

Como vimos na seção 3.7, os eventos de Monte Carlo para os backgrounds têm baixa eficiência quando cortes retangulares duros são aplicados. Porém esses eventos possuem uma grande seção de choque associada, levando à uma grande produção de eventos para uma dada luminosidade integrada L. Na Fig. 3.22, mostramos todas as distribuições usadas na análise para supersimetria, MUED e background com o conjunto Cortes Ótimos aplicados. O efeito da estatística limitada compromete a suavidade das distribuições cinemáticas para o background. Por isso a necessidade de modelar uma incerteza sistemática teórica associada ao número de eventos de Monte Carlo que efetivamente restam após os cortes retangulares [71]. Realizamos uma análise para verificar o impacto da incerteza sistemática teórica no formato das distribuições dos backgrounds. Na Fig. 3.23 temos a análise para o caso de descoberta de supersimetria. No caso onde não temos incertezas sistemáticas no formato das distribuições (curvas azuis), temos um resultado mais otimista para o alcance da descoberta de supersimetria. Portanto, inserir uma incerteza sistemática na forma das distribuições, além de trazer o benefício de modelagem da baixa estatística de Monte Carlo após cortes duros para os backgrounds, não superestima a descoberta de supersimetria. 

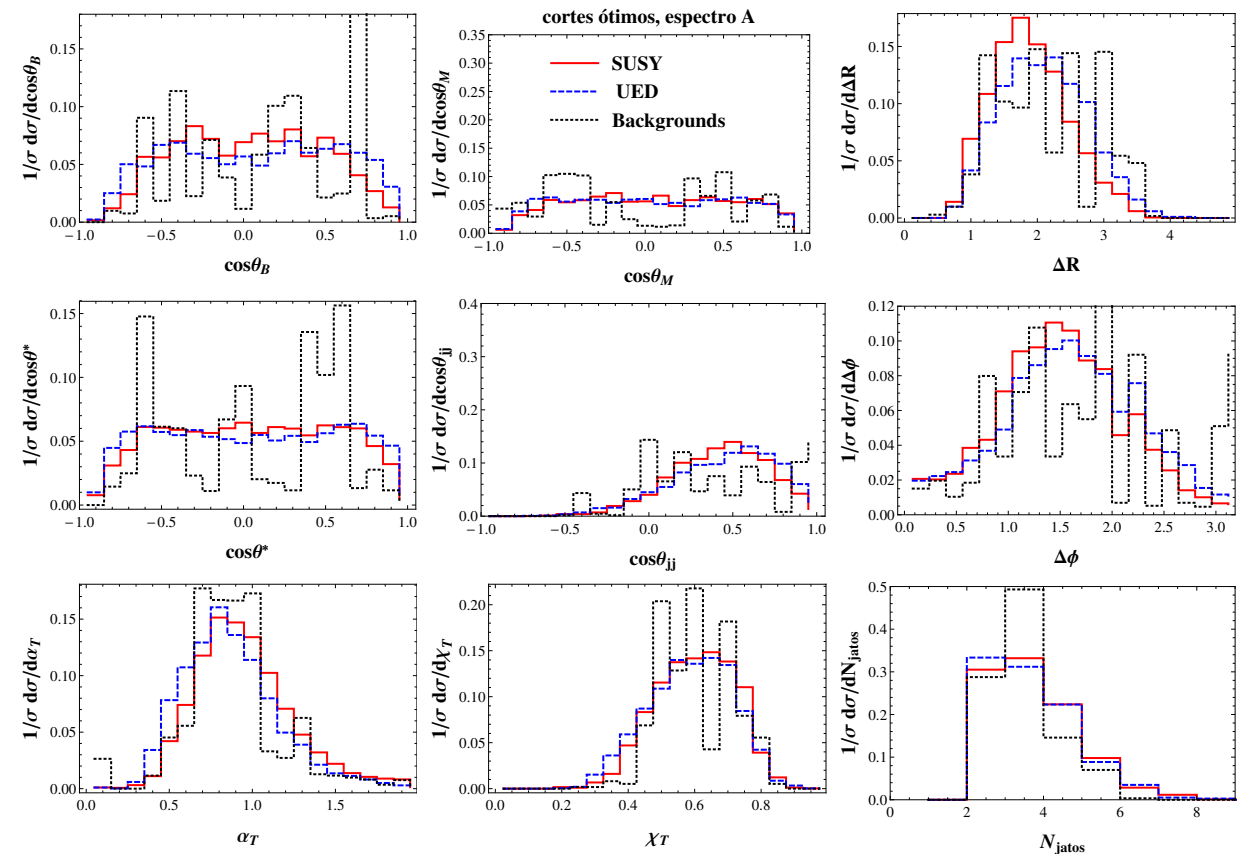

Figura 3.22: Distribuições normalizadas para supersimetria, MUED e Modelo Padrão. Após aplicação do conjunto de cortes Cortes Ótimos.

\subsection{A escolha do tagging de jatos de quarks e glúons}

Para definir o melhor tagging de jatos fizemos uma análise com os valores de significância para a estatística log-likelihood-ratio. Para descoberta de supersimetria, consideramos três cenários diferentes para luminosidade integrada, Todos Sistemáticos com $\epsilon_{\text {taxa }}=20 \%$ e Cortes Ótimos. Na Fig. 3.24 temos curvas para essa análise, em função do tagging de jatos de quarks $\epsilon_{q}$. Ressaltamos que $\epsilon_{g}$ depende de $\epsilon_{q}$, como vimos na seção 3.11, porém um dos dois valores pode ser escolhido de forma arbitrária.

Colocamos um eixo adicional paralelo a $\epsilon_{q}$, que denota a exclusão de glúons $\operatorname{tag}_{g}=\frac{1}{\epsilon_{g}}$. Os valores de $\epsilon_{q}$ e $\epsilon_{g}$ são os fatores que modulam as seções de choque dos canais de produção com quarks e glúons, veja a seção 3.12 . Vemos que $Z_{L L R}$, de fato, é beneficiada com a aplicação do tagging de jatos. Observe que o aumento da luminosidade integrada faz com o que $\epsilon_{q}$ seja cada vez menor, e isso portanto, aumenta a exclusão de glúons. Esse comportamento é esperado, pois grandes luminosidades possibilitam a retirada maior de glúons e quarks sem prejudicar a métrica $Z_{L L R}$.

Para cada valor de luminosidade integrada, temos um tagging de jatos de quarks e glúons que maximiza a descoberta de supersimetria para $Z_{L L R}$. Note que para $\epsilon_{q}=\epsilon_{g}=1.0$, temos praticamente significâncias idênticas para os três valores de luminosidade integrada, isso deve-se à saturação da seção de choque com o erro sistemático $\epsilon_{\text {taxa }}$ que observamos na equação $2.46 \mathrm{e}$ na Fig. 3.20,

Com a ajuda dos resultados da Fig. 3.24, definimos as relações de luminosidade integrada e tagging de jatos de quarks que mostramos em 3.14 .

- $L=100 \mathrm{fb}^{-1}$, nesse caso $\epsilon_{q}=0.53$ e $\epsilon_{g}=\frac{1}{11}$.

- $L=500 \mathrm{fb}^{-1}$, nesse caso $\epsilon_{q}=0.38$ e $\epsilon_{g}=\frac{1}{41}$.

- $L=3000 \mathrm{fb}^{-1}$, nesse caso $\epsilon_{q}=0.32$ e $\epsilon_{g}=\frac{1}{59}$.

Realizamos uma análise para observar o impacto do tagging na contagem de eventos. Criamos curvas de níveis para a razão $\frac{s}{b}$, onde $s$ e $b$ são os números de eventos totais de sinal e background 


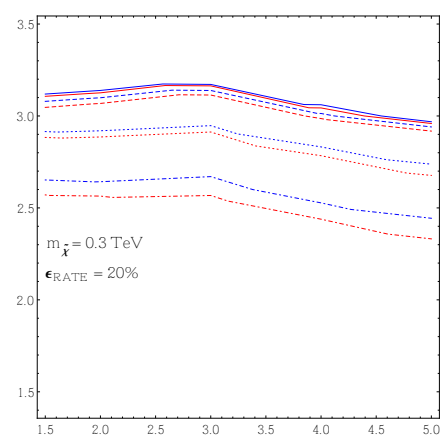

(a)

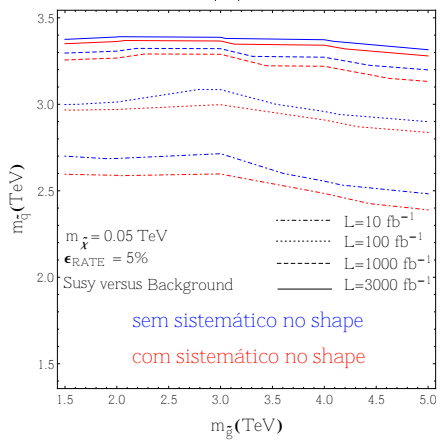

(c)

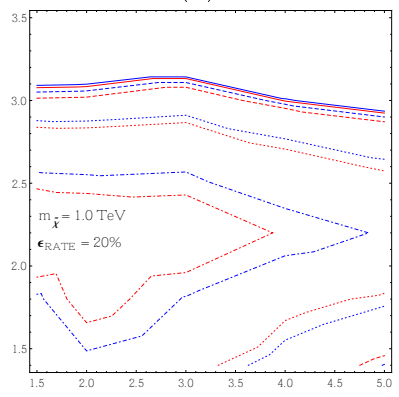

(e)

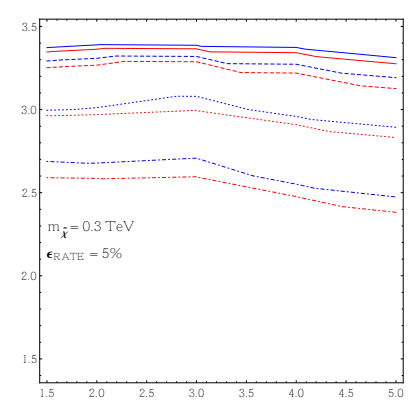

(b)

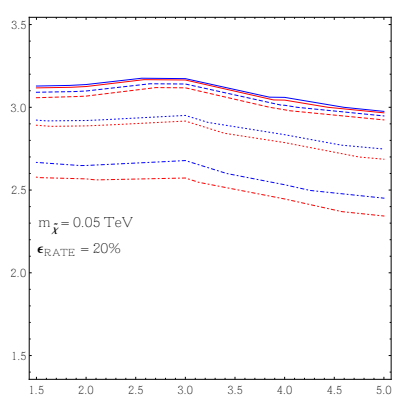

(d)

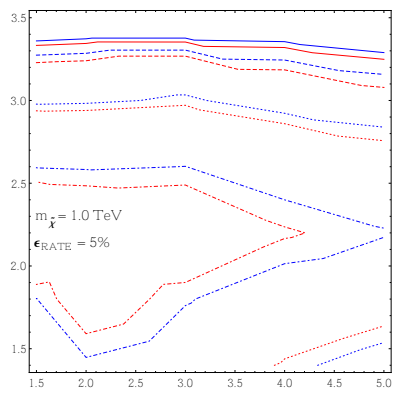

(f)

Figura 3.23: Análise do impacto da incerteza sistemática devido à escassez de eventos de Monte Carlo para os backgrounds. Esse efeito é inserido através de um erro sistemático no formato das distribuições dos backgrounds.

em função de $m_{\tilde{q}}$ e $m_{\tilde{g}}$. Na Fig. 3.25 temos dois gráficos que mostram o impacto do tagging de jatos na contagem de eventos. Podemos perceber que a aplicação de tagging de jatos aumenta as regiões de $\frac{s}{b}$ iguais a 0.1 e 1 .

Isso é esperado, porque, no caso da Fig. 3.25, a seção de choque para o sinal tem uma redução de $50 \%$ enquanto que os backgrounds de $95 \%$, após aplicação do tagging de jatos, veja seção 3.11 . Criamos também a Fig. 3.26, que é um análogo à Fig. 3.24, porém, para a contagem de eventos temos a inclusão somente de um erro sistemático na taxa dos eventos de background. Observe que a contagem de eventos (Fig. 3.26 tem comportamento bem semelhante ao visto na análise multivariada (Fig. 3.24).

Finalmente analisamos o impacto da incerteza sistemática $\epsilon_{\text {taxa }}$ no tagging de jatos de quarks e glúons. Na Fig. 3.27 vemos que o tagging de jatos tem impacto diferente nos testes $Z_{L L R}$ e $Z_{s b}$. Existe uma região onde a métrica de significância para $\epsilon_{\text {taxa }}=5,10,20 \%$ converge, $0.1<\epsilon_{q}<0.4$ para $Z_{L L R}$ e $0.1<\epsilon_{q}<0.2$ para $Z_{s b}$. Nessas regiões ocorre o decréscimo acentuado do número de eventos de background devido à grande exclusão advinda do tagging de jatos, tornando a métrica de significância praticamente independente de flutuações dos backgrounds devido ao erro 


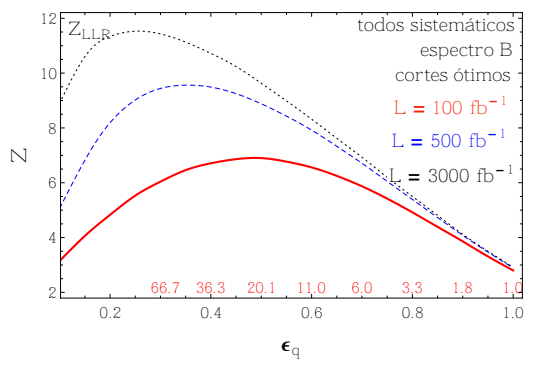

Figura 3.24: Análise para determinação do melhor tagging de jatos de quarks para descoberta de supersimetria.

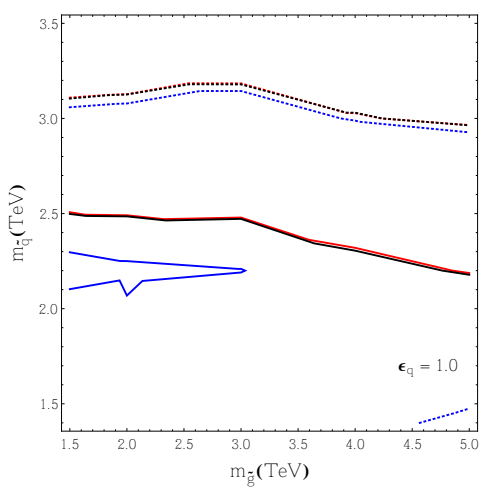

(a)

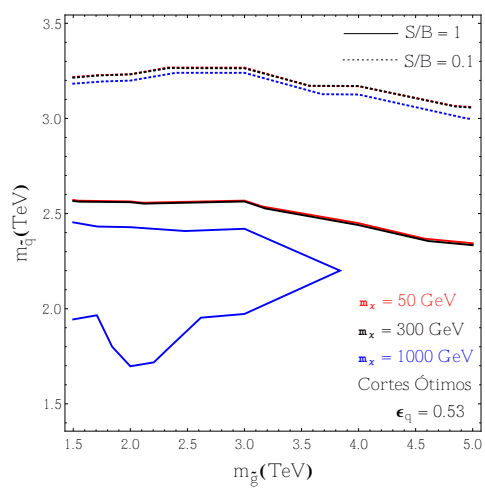

(b)

Figura 3.25: Impacto da aplicação de tagging de jatos para $\frac{s}{b}$. Há um aumento de todas as regiões delimitadas pelas curvas de nível quando aplicamos o tagging de jatos.

sistemático $\epsilon_{\text {taxa }}$. Note que essa região de independência do erro sistemático $\epsilon_{\text {taxa }}$ ocorre antes para $Z_{L L R}$, porque essa métrica tem uma sensibilidade maior aos erros sistemáticos.

Ainda na Fig. 3.27, vemos que o tagging não beneficia tanto $Z_{s b}$, o máximo dessas curvas acontece em aproximadamente $0.8<\epsilon_{q}<1$. Esse comportamento pode ser entendido com a equação (2.46) e a Fig. 2.11). Para um erro sistemático fixo, não é qualquer aumento da razão $s / b$ que leva a um acréscimo em $Z_{s b}$. Por isso é esperado que $Z_{s b}$ não se beneficie muito com qualquer tagging de jatos.

Ressaltamos também que, embora não explicitado, na Fig. 3.27, existem diferenças pequenas para os casos de $m_{\tilde{\chi}_{1}}$ de 50 e $300 \mathrm{GeV}$. Os dois valores de massas fornecem praticamente a mesma razão $\frac{S}{b}$, porque têm seções de choque e eficiência de cortes muito parecidas. Portanto esboçamos somente o caso de $m_{\tilde{\chi} 1}=300 \mathrm{GeV}$. 


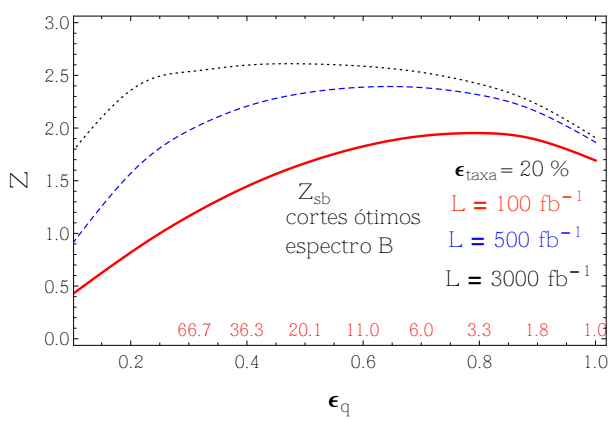

Figura 3.26: Análise referência para o comportamento do tagging de jatos em função da significância para a contagem de eventos.

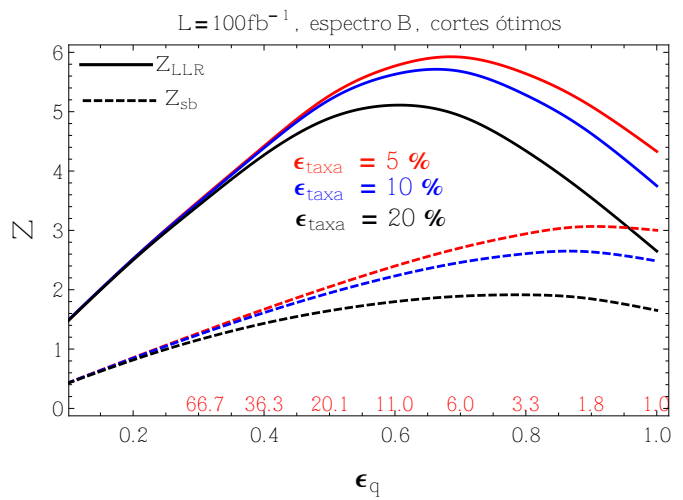

Figura 3.27: Influência das incertezas sistemáticas na análise de tagging de jatos para os testes $Z_{L L R}$ e $Z_{s b}$. 


\section{Capítulo 4}

\section{Resultados}

Esse capítulo será dedicado à exposição e verificações dos resultados obtidos. Apresentaremos nossos resultados em um espaço de massas, $\left\{m_{\tilde{q}}, m_{\tilde{g}}\right\}$ e $m_{\tilde{\chi}_{1}}$, dependendo do tipo de análise. Nesse espaço serão esboçadas curvas de níveis, referentes às métricas de significância iguais a $5 \sigma$, para o teste de hipóteses. Temos, assim, que essas curvas de $5 \sigma$ sempre levam à exclusão da hipótese nula como melhor representante dos dados simulados e observados. Na Fig. 4.1 temos um exemplo de uma curva de $5 \sigma$ para nossa análise, ela exemplifica como são representadas nossas regiões de descoberta e discernimento.

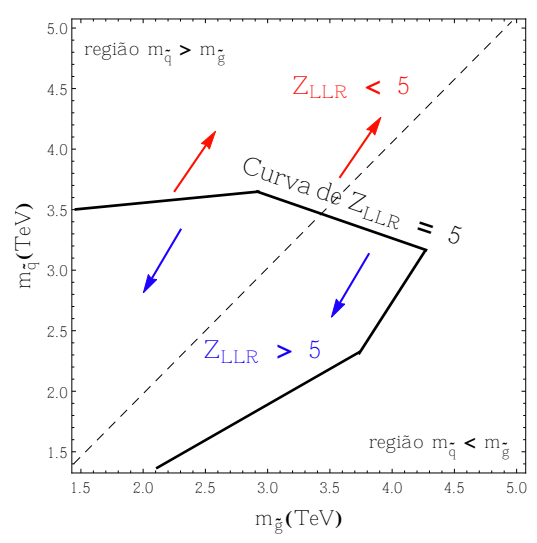

Figura 4.1: Figura exemplo para interpretação de nossos resultados no plano de massas $\left\{m_{\tilde{q}}, m_{\tilde{g}}\right\}$. Destacamos aqui a região indicada pelas setas azuis, ela indicam pontos para massas de squarks e gluinos onde é possível alcançar significância maior que $5 \sigma$, enquanto que as regiões com a seta vermelha o contrário. As regiões onde $m_{\tilde{q}}>m_{\tilde{g}}$ e $m_{\tilde{q}}<m_{\tilde{g}}$ também desempenham papel importante na interpretação dos resultados, como veremos adiante.

Os modelos que estudamos assumem um certo conjunto de condições, como já discutimos nos capítulos 2 e 3 desse trabalho. Para supersimetria e MUED estamos assumindo que:

- $B R(\tilde{g} \rightarrow \tilde{q}+q)=B R\left(g^{(1)} \rightarrow q^{(1)}+q\right)=100 \%$.

- $B R\left(\tilde{q}_{R} \rightarrow q+\tilde{\chi}_{1}\right)=B R\left(q^{(1)} \rightarrow q+B^{(1)}\right)=99 \%$.

- $B R\left(\tilde{q}_{L} \rightarrow q+\tilde{\chi}_{1}\right)=B R\left(Q^{(1)} \rightarrow q+B^{(1)}\right)=1 \%$.

- As seções de choque de MUED são normalizadas às seções de choque de supersimetria.

- Não estamos assumindo correlações estatísticas entre os observáveis físicos usados na análise. 


\subsection{Discussão dos resultados no espaço de massas}

Podemos investigar como uma análise multivariada com nove observáveis sensíveis à diferentes características dos modelos de SUSY e MUED, usando a estatística-teste log-likelihood ratio, com a aplicação de incertezas sistemáticas (tabela 3.5), pode fornecer resultados sobre a descoberta e discernimento de modelos como supersimetria e MUED no LHC $14 \mathrm{TeV}$. Além disso, podemos estimar os limites que o LHC alcançará em uma análise fenomenológica mais realista, pois leva em conta incertezas sistemáticas de diversas origens.

Nosso trabalho possui enfoque no regime de grande luminosidade integrada $L: 100,500$ e $3000 \mathrm{fb}^{-1}$. O resultado será apresentado através de curvas de nível para valores de $Z_{L L R}$, em um espaço bi-dimensional $\left\{m_{\tilde{q}}, m_{\tilde{g}}\right\} \operatorname{com} m_{\tilde{\chi} 1}$ fixo caso a caso. Consideramos três cenários diferentes de incertezas sistemáticas, o Todos Sistemáticos onde $\epsilon_{\text {taxa }}=0,10$ e $20 \%$ e três massas de neutralino (kk-fotons) diferentes, 50, 300 e $1000 \mathrm{GeV}$. Todos analisados no conjunto de cortes retangulares Cortes Ótimos 3.14. É importante ter em mente como definimos o valor observado da estatística-teste $\left(\Lambda_{\mathrm{OBS}}\right)$ no teste de hipóteses 2.8 , usando a mediana da hipótese alternativa.

Alguns resultados observados serão explicados, pormenorizadamente, na próximas seções.

\subsubsection{Descoberta de supersimetria}

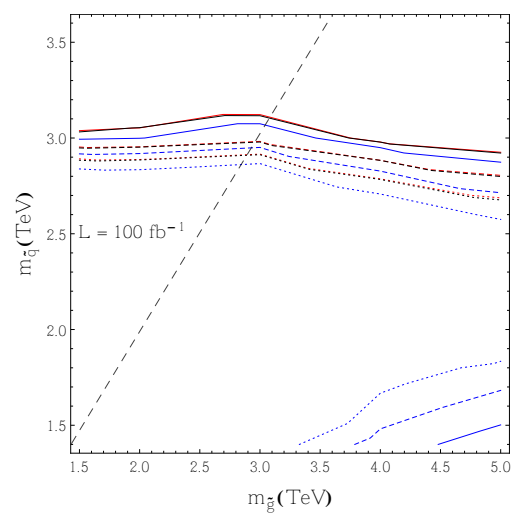

(a)

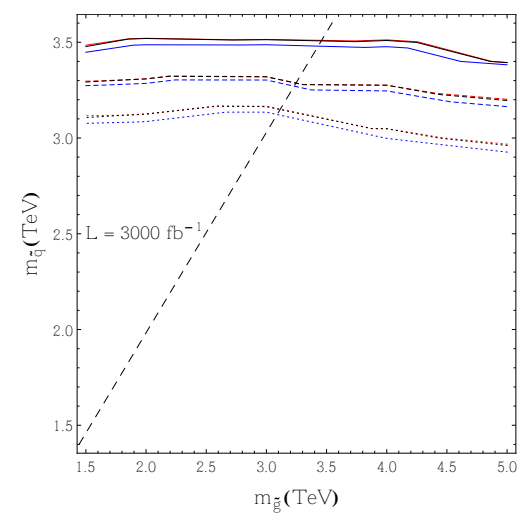

(b)

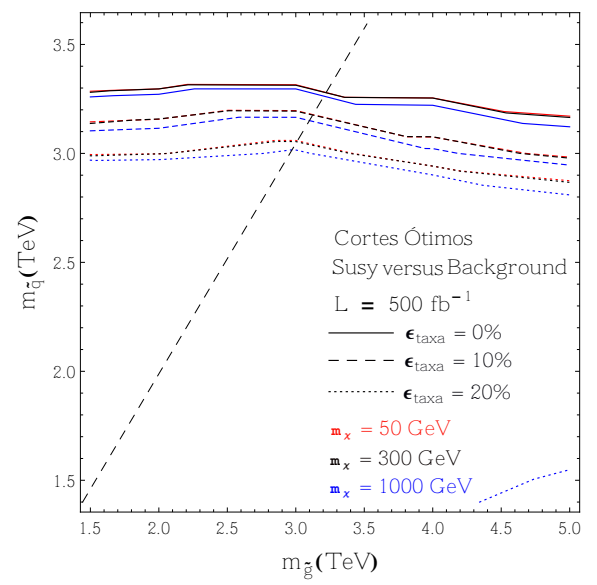

(c)

Figura 4.2: Curvas de nível $Z_{L L R}=5$, para descoberta de supersimetria. Vemos que para $L=3000 \mathrm{fb}^{-1}$, podemos alcançar descoberta de supersimetria para squarks de massas de até 3.1 $\mathrm{TeV}$ com incertezas sistemáticas na taxa dos backgrounds de $20 \%$. O real limite para a massa dos gluinos não fica claro, pois até onde simulamos, $5 \mathrm{TeV}$, são regiões de massa facilmente acessíveis para descoberta. 
Na Fig. 4.2 temos três curvas de nível para a significância da estatística log-likelihood ratio $\left(Z_{L L R}\right)$ em função de $m_{\tilde{q}}$ e $m_{\tilde{g}}$.

1. Podemos notar que as curvas para $m_{\tilde{\chi}_{1}}$ de 50 e $300 \mathrm{GeV}$ estão, aproximadamente, sobrepostas. Mostrando que, para descoberta de supersimetria, nossa análise multivariada possui pouca sensibilidade para diferenças de massas de neutralino de algumas centenas de GeV. Já que o formato das distribuições cinemáticas (vide a seção 4.3), o número de eventos que passam nos cortes retangulares, as seções de choque de produção supersimétricas e as incertezas da escala de fatorização, para neutralinos de 50 e $300 \mathrm{GeV}$, são parecidas.

2. Observamos uma clara dependência da região de descoberta com o erro sistemático $\epsilon_{\text {taxa }}$, vide a seção 3.13 . Para uma luminosidade integrada de $100 \mathrm{fb}^{-1}$, as regiões de descoberta tornam-se menos sensíveis a $\epsilon_{\text {taxa }}$, quando comparadas com as curvas de $3000 \mathrm{fb}^{-1}$. Esse comportamento pode ser entendido usando a equação (2.42), nela vemos que quanto maior o número de eventos de background, $b=\sigma_{\text {back }} \cdot L$, maior é a chance de uma flutuação estatística do número de eventos de backgrounds, o que acarreta na diminuição da influência dos eventos de supersimetria. Essa conclusão é análoga para a análise multivariada usando likelihoods, com a adicional ressalva de que as incertezas sistemáticas no formato das distribuições de backgrounds influenciam também em $Z_{L L R}$, como vimos na seção 3.16 .

3. A região de transição de $m_{\tilde{q}}>m_{\tilde{g}}$ para $m_{\tilde{q}}<m_{\tilde{g}}$, delimitada pela linha transversal pontilhada, mostra-se com curvas de inclinação ligeiramente decrescentes, na parte superior, com relação à parte inferior. Essa influência é geral em nossos resultados, e é decorrente da subtração dos canais que não contribuem com jatos e MET nesse regime, os de produção associada de gluinos, veja seção 3.5.1. Isso diminui consideravelmente as contribuições de eventos para o sinal de supersimetria na região $m_{\tilde{q}}>m_{\tilde{g}}$, consequentemente, diminuindo o poder de descoberta de supersimetria.

4. Destacamos que as curvas para $m_{\tilde{\chi}_{1}}=1 \mathrm{TeV}$ possuem as menores regiões de descoberta de supersimetria. Isso porque o $p_{T}$ dos jatos produzidos no decaimento de um squark em neutralino pesado são baixos em comparação com os casos de $m_{\tilde{\chi}_{1}}=50$ e $300 \mathrm{GeV}$, embora $\not_{T}$ seja alto. Assim, menos eventos passam nos cortes retangulares definidos pelo conjunto Cortes Ótimos, veja seção cenários 3.14

5. Em relação à descoberta, squarks de até $3.5 \mathrm{TeV}$ estão acessíveis (Fig. 4.2b), enquanto que o limite para gluinos pode superar os $5 \mathrm{TeV}$. Isso decorre de, principalmente, dois fatores: primeiro, as altas incertezas sistemáticas associadas à escala de fatorização do sinal para massas de squarks grandes, veja seção 3.10 . Segundo, e mais relevante, a região onde $m_{\tilde{q}}<m_{\tilde{g}}$ tem contribuições para o sinal vindas de canais de produção de squarks e gluinos, e nessa região, a seção de choque de produção para squarks supera a de produção de gluinos. Enquanto que a outra região tem contribuições apenas de squarks, e aqui, a produção de squarks é secundária, ou seja, os canais que mais participam na região $m_{\tilde{q}}>m_{\tilde{g}}$, os de gluinos, devem ser retirados, e os que menos contribuem são os únicos que restam para a análise de jatos e MET, veja Fig. 3.4 e subseção 3.5.1. Note que a descoberta deve ser entendida no sentido conjunto, ou seja, onde squarks e gluinos participam dos eventos de nova física, ainda que a contribuição de squarks seja a dominante na maior parte do espaço de massas acessível à descoberta.

As curvas de $\epsilon_{\text {taxa }}=0 \%$ são as que cobrem as maiores regiões do espaço de massas, o que já era esperado, pois um cenário de ausência de todo e qualquer erro sistemático seja impossível. Podemos descobrir supersimetria para $m_{\tilde{q}}<3.0 \mathrm{TeV}$ e $m_{\tilde{g}}<5.0 \mathrm{TeV} \operatorname{com} L=100 \mathrm{fb}^{-1}$. Quando $L=3000 \mathrm{fb}^{-1}$, há um aumento dessa região em, aproximadamente, $500 \mathrm{GeV}$ na massa dos squarks, nesse caso, o novo limite de descoberta é $m_{\tilde{q}}<3.5 \mathrm{TeV}$. Observamos um decréscimo de aproximadamente $50 \mathrm{GeV}$ para a região de descoberta de supersimetria quando $m_{\tilde{\chi}_{1}}=1 \mathrm{TeV}$ e 
notamos que a Fig. $4.2 \mathrm{a}$ possui um região para $m_{\tilde{q}}=1.5 \mathrm{TeV}$ e $m_{\tilde{g}}>4.5 \mathrm{TeV}$ onde não é possível alcançar descoberta de supersimetria para $m_{\tilde{\chi}_{1}}=1 \mathrm{TeV}$. Como já dissemos, esses espectros, com $m_{\tilde{q}}$ baixo e $m_{\tilde{\chi}_{1}}$ alto, têm eventos que não passam nos cortes retangulares, porque geram jatos com baixo $p_{T}$.

Para as curvas de $\epsilon_{\text {taxa }}=10(20) \%$ a descoberta de supersimetria cai para $m_{\tilde{q}}<2.9(2.8) \mathrm{TeV}$ para $L=100 \mathrm{fb}^{-1}$ (Fig. $4.2 \mathrm{a}$ ) e $m_{\tilde{q}}<3.3(3.1) \mathrm{TeV}$ para $L=3000 \mathrm{fb}^{-1}$ (Fig. $4.2 \mathrm{~b}$ ). Novamente temos uma região na parte inferior da Fig. $4.2 \mathrm{a}\left(m_{\tilde{q}}<m_{\tilde{g}}\right)$ onde o decaimento de neutralinos, de $1 \mathrm{TeV}$, não permitem descoberta de supersimetria, agora essa região é, para $100 \mathrm{fb}^{-1}$, de $m_{\tilde{q}}<1.6(1.7) \mathrm{TeV}$ e $m_{\tilde{g}}>3.8(3.3) \mathrm{TeV}$. No regime de $3000 \mathrm{fb}^{-1}$, essa região deixa de existir (regime $m_{\tilde{q}}<m_{\tilde{g}}$ da Fig. 4.2b), e agora a alta luminosidade compensa a perda de eventos vindos do decaimento de squarks em um neutralino de $m_{\tilde{\chi}_{1}}=1 \mathrm{TeV}$, permitindo a descoberta de supersimetria para $\epsilon_{\text {taxa }}=20 \%$.

\subsubsection{Discernimento de supersimetria e MUED}

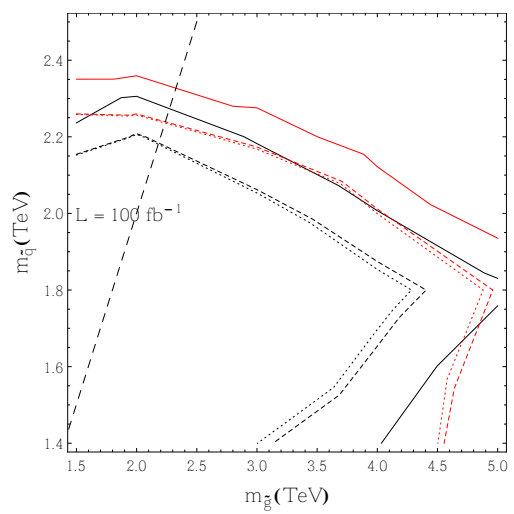

(a)

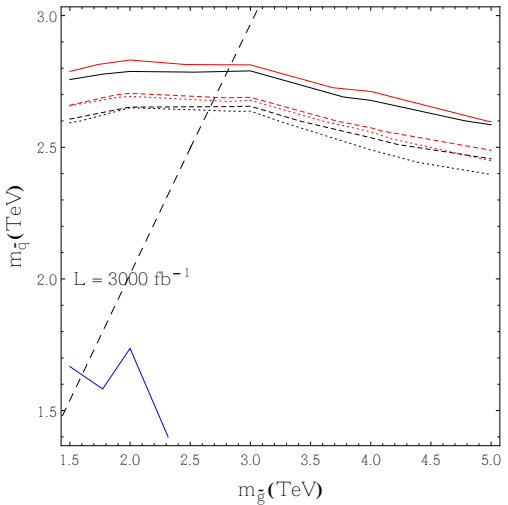

(b)

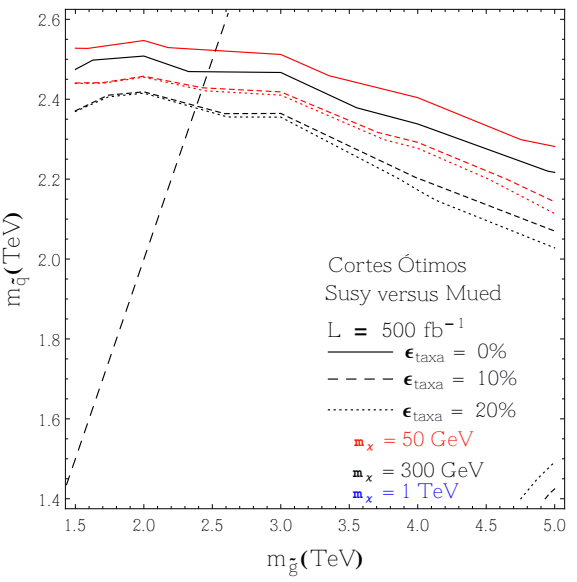

(c)

Figura 4.3: Curvas de nível $Z_{L L R}=5$ para discernimento entre supersimetria e MUED. Primeiramente vemos que as curvas de $5 \sigma$ para matéria escura de $1 \mathrm{TeV}$ são, praticamente, inexistentes. Pode-se alcançar discernimento de supersimetria e MUED para squarks (KK-quarks) de até 2.60 $\mathrm{TeV}$ para incertezas sistemáticas na taxa dos eventos de backgrounds de $20 \%$ e luminosidade de $3000 \mathrm{fb}^{-1}$. Pra gluinos e KK-glúons esse limite é de $\sim 5 \mathrm{TeV}$, para o mesmo valor de erro sistemático e luminosidade integrada.

Na Fig. 4.3 temos as curvas de $Z_{L L R}=5$ para o discernimento entre supersimetria e MUED. Ressaltamos que supersimetria e backgrounds estão associados à hipótese nula $\left(H_{0}\right)$, de modo que a região de massas dentro das curvas de $5 \sigma$ denotam que o modelo de supersimetria pode 
ser discernido de um modelo de MUED e backgrounds. Enquanto que nas regiões externas das curvas de $Z_{L L R}=5$ (Fig. 4.1) o discernimento não é possível neste nível de significância. Regiões de $95 \%$ ou $99 \%$ de nível de confiança devem abarcar áreas bem maiores do espaço de massas. É interessante notar que recentes avanços no estudo do spin e paridade do bóson de Higgs, veja [70], aproveitam-se também de testes de hipóteses, onde confrontam-se uma partícula de spin-0 e CPpar, o bóson de Higgs advindo do Modelo Padrão, contra algum bóson de Higgs de outro modelo com propriedades diferentes, porém de mesma massa. Alguns desses resultados são apresentados, hoje, com níveis de confiança de $99 \%$, algo em torno de $3 \sigma$. Nossos cenários de análise requerem configurações mais rigorosas, muito adequadas a uma análise teórica dos limites do acelerador LHC.

1. O que podemos observar, de imediato, é que apenas uma curva para $m_{\tilde{\chi}_{1}}=1 \mathrm{TeV}$ aparece nos gráficos de discernimento, o da Fig. 4.3b, referente a $L=3000 \mathrm{fb}^{-1}$ e $\epsilon_{\text {taxa }}=0 \%$. É possível discriminar entre os modelos de supersimetria e MUED somente no regime improvável de incertezas sistemáticas muito pequenas, quando a luminosidade integrada é menor que alguns milhares de $\mathrm{fb}^{-1}$. Isso está relacionado com a quantidade de eventos de Monte Carlo, para $m_{\tilde{\chi}_{1}}=1 \mathrm{TeV}$, que não passam no conjunto Cortes Ótimos, vide 4.2 . Relaxando os cortes, especificamente para essa massa de matéria escura, poderia levar a resultados melhores nesse regime.

2. O discernimento é sensível à mudanças de algumas centenas $\mathrm{GeV}$ para a massa no neutralino (KK-fóton), observe as curvas pretas e vermelhas na Fig. 4.3. As curvas vermelhas, $m_{\tilde{\chi}_{1}}=50 \mathrm{GeV}$, são aquelas onde o discernimento de modelos é mais fácil, devido à produção de jatos mais duros, para essa massa, e com isso maior aceitação após cortes retangulares.

3. O alcance do discernimento, em termos absolutos, é bem menor do que a descoberta de supersimetria para a massa dos squarks. No caso de maior luminosidade integrada e sem sistemáticos, esse alcance é de no máximo $2.7 \mathrm{TeV}$, embora ainda seja possível observar discernimento em cenários de gluinos de até $5 \mathrm{TeV}$. Para os squarks, isso é reflexo do erro sistemático na escala de fatorização do sinal, agora, ele atinge as duas hipóteses, veja 3.10. Além disso, a região de $m_{\tilde{q}}>m_{\tilde{g}}$ tem apenas um canal para produção de jatos e MET.

4. A região de transição entre $m_{\tilde{q}}<m_{\tilde{g}}$ e $m_{\tilde{q}}>m_{\tilde{g}}$ apresenta comportamento idêntico à descoberta de supersimetria (seção 4.1.1 item 3), exatamente pelo mesmo motivo, a subtração de canais que contribuem de forma relativamente alta na produção de jatos e MET, devido à hierarquia de massas. A diferença é que as curvas de discernimento concentram-se mais na região $m_{\tilde{q}}<m_{\tilde{g}}$ (abaixo da linha tracejada), denotando que discernir modelos é um procedimento mais sensível à ausência dos canais de gluinos na parte superior dos gráficos.

5. Destacamos a dependência dos erros sistemáticos também na discriminação de modelos, como esperado. Na região $m_{\tilde{q}}>m_{\tilde{g}}$, existe uma dependência menor do discernimento com o erro sistemático $\epsilon_{\text {taxa }}$ para $m_{\tilde{\chi}_{1}}$ fixo, as curvas de 10 e $20 \%$ para esse erros ficam bem próximas umas das outras. Isso pode ser entendido retomando a equação 2.39 para a estatística-teste do discernimento de hipóteses. Essa equação tem os backgrounds incluídos na hipótese nula e alternativa, embora sejam feitos sorteios independentes gaussianos, a incerteza sistemática no background acaba tornando-se uma espécie de efeito global e dominante, já que nessa região a contribuição vem somente de produção de squarks pesados. Já na região $m_{\tilde{q}}<m_{\tilde{g}}$, temos também um efeito pequeno com a mudança de $\epsilon_{\text {taxa }}$, porém ainda assim é possível notar diferenças entre as curvas, isso porque nessa região, a contribuição de sinal é maior do que na parte superior da linha transversal tracejada, tornando assim, o efeito do sistemático nas duas hipóteses, levemente menos dominante do que no primeiro caso. 
6. É nítido um efeito de baixa luminosidade integrada na Fig. 4.3a a região de $m_{\tilde{g}}>3.0 \mathrm{TeV}$ e $m_{\tilde{q}}=1.8 \mathrm{TeV}$, apresenta um regime onde não é possível discernir modelos de supersimetria e MUED. Ressaltamos que esse comportamento não ocorre quando $L=3000 \mathrm{fb}^{-1}$, mostrando que esses espectros têm um baixo número de eventos de supersimetria, e consequentemente de MUED, já que as seções de choque de produção do sinal, nesse regime, são da ordem de $10^{-3} \mathrm{fb}$, veja Fig. 3.4 .

Tabela 4.1: Regiões de possível discernimento para $m_{\tilde{\chi}_{1}}=50 \mathrm{GeV}$ (Fig. 4.3.

\begin{tabular}{|l|c|c|c|}
\hline & $\mathrm{L}\left(\mathrm{fb}^{-1}\right)$ & Região $m_{\tilde{q}}(\mathrm{TeV})$ & Região $m_{\tilde{g}}(\mathrm{TeV})$ \\
\hline \multirow{3}{*}{ Sem Sistemáticos } & 100 & $2.00(2.35)$ & $5.00(5.00)$ \\
& 500 & $2.35(2.55)$ & $5.00(5.00)$ \\
& 3000 & $2.70(2.80)$ & $5.00(5.00)$ \\
\hline \multirow{3}{*}{$\epsilon_{\text {taxa }}=10 \%$} & 100 & $1.80(2.20)$ & $4.55(4.90)$ \\
& 500 & $2.20(2.45)$ & $5.00(5.00)$ \\
$\epsilon_{\text {taxa }}=20 \%$ & 3000 & $2.45(2.65)$ & $5.0(5.00)$ \\
& 100 & $1.80(2.20)$ & $4.50(4.80)$ \\
& 500 & $2.15(2.45)$ & $5.00(5.00)$ \\
& 3000 & $2.45(2.65)$ & $5.00(5.00)$ \\
\hline
\end{tabular}

Tabela 4.2: Regiões de possível discernimento para $m_{\tilde{\chi}_{1}}=300 \mathrm{GeV}$ (Fig. 4.3.

\begin{tabular}{|l|c|c|c|}
\hline & $\mathrm{L}\left(\mathrm{fb}^{-1}\right)$ & Região $m_{\tilde{q}}(\mathrm{TeV})$ & Região $m_{\tilde{g}}(\mathrm{TeV})$ \\
\hline \multirow{3}{*}{ Sem Sistemáticos } & 100 & $1.85(2.25)$ & $4.00(5.00)$ \\
& 500 & $2.25(2.45)$ & $5.00(5.00)$ \\
& 3000 & $2.70(2.75)$ & $5.00(5.00)$ \\
\hline \multirow{3}{*}{$\epsilon_{\text {taxa }}=10 \%$} & 100 & $1.80(2.15)$ & $3.10(4.40)$ \\
& 500 & $2.10(2.35)$ & $4.90(5.00)$ \\
& 3000 & $2.45(2.60)$ & $5.0(5.00)$ \\
$\epsilon_{\text {taxa }}=20 \%$ & 100 & $1.80(2.15)$ & $3.00(4.30)$ \\
& 500 & $2.05(2.35)$ & $4.70(5.00)$ \\
& 3000 & $2.40(2.60)$ & $5.00(5.00)$ \\
\hline
\end{tabular}

Construímos as tabelas $4.1 \mathrm{e} 4.2$, na qual sintetizamos os limites impostos para as massas de squarks (KK-quarks) e gluinos (KK-glúons) de acordo com a luminosidade, erros sistemáticos e $m_{\tilde{\chi}_{1}}$. Nessas tabelas temos os alcances das massas dos squarks e gluinos exibidos através de valores mínimos e máximos. Esses limites circunscrevem regiões de discernimento.

\subsubsection{Descoberta e posterior discernimento de nova física}

A análise derradeira do nosso trabalho ocorre quando combinamos os resultados obtidos na descoberta e discernimento. A discriminação de modelos é um passo secundário, realizado depois da confirmação que um determinado conjunto de eventos, de fato, contém informações de nova física. Assim as regiões de discernimento de nova física só são validadas se constatarmos que também pode-se alcançar descoberta nessas regiões. Para isso criamos a Fig. 4.4, na qual referese à apenas cenários de $L=100$ e $3000 \mathrm{fb}^{-1}$, com $m_{\tilde{\chi}_{1}}=50$ e $300 \mathrm{GeV}$, excluímos os resultados para $m_{\tilde{\chi}_{1}}=1 \mathrm{TeV}$ pelas razões discutidas na seção anterior. As incertezas sistemáticas usadas 
seguem o conjunto, Todos Sistemáticos, com $\epsilon_{\text {taxa }}=20 \%$ e conjunto Cortes Ótimos foi aplicado. As curvas contínuas denotam a descoberta de supersimetria e foram retiradas da Fig. 4.2 e as curvas tracejadas referem-se ao discernimento de supersimetria e MUED e foram obtidas da Fig. 4.3, apropriadamente. A região destacada na Fig. 4.4 denota o alcance real da análise multivariada usada nesse trabalho, a chamaremos de região descoberta-discernimento. Essa região é completamente descrita pelas curvas de discernimento, Fig. 4.3 , pois a descoberta de supersimetria engloba toda essa região. Portanto, as observações e limites estimados na seção 4.1 .2 são os mesmos para os limites de descoberta de supersimetria e discernimento desse modelo com relação ao modelo de MUED. Com isso os limites para região descoberta-discernimento coincidem com os discutidos nas tabelas $4.1 \mathrm{e} 4.2$, para $m_{\tilde{\chi}_{1}}=50,300 \mathrm{GeV}$, respectivamente.

Estima-se que em 2016 o LHC conseguirá funcionar de acordo com suas especificações projetadas, a partir disso espera-se que a aquisição de dados alcance $50 \mathrm{fb}^{-1}$ em pouco mais de um semestre de funcionamento. Em 2020 estima-se uma luminosidade integrada de $300 \mathrm{fb}^{-1}$, com a aquisição anual de $100 \mathrm{fb}^{-1}$ a partir de então, veja [101].

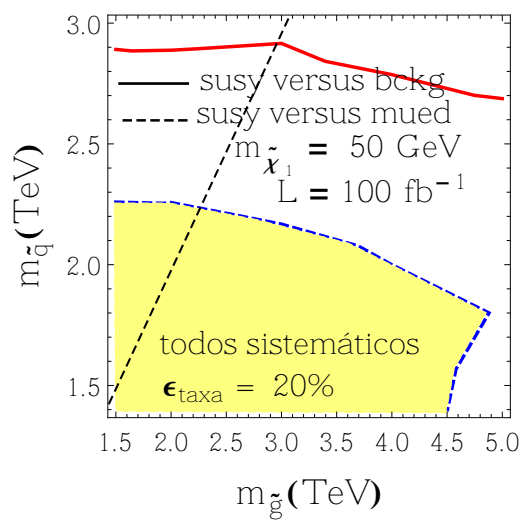

(a)

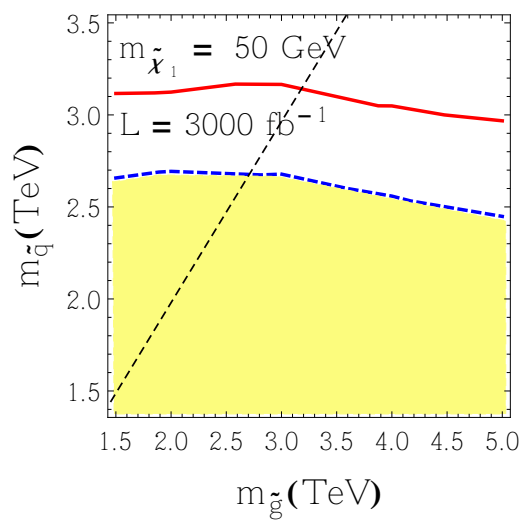

(c)

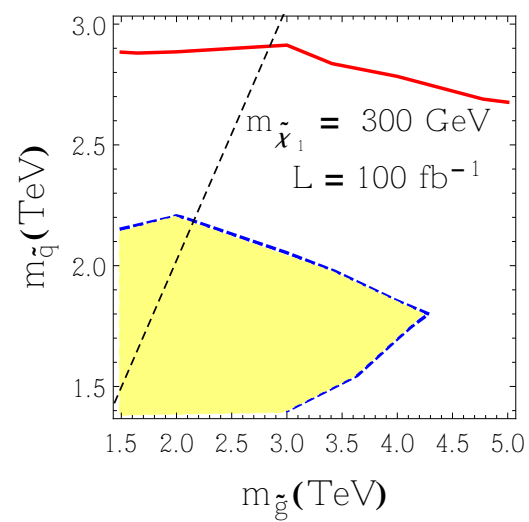

(b)

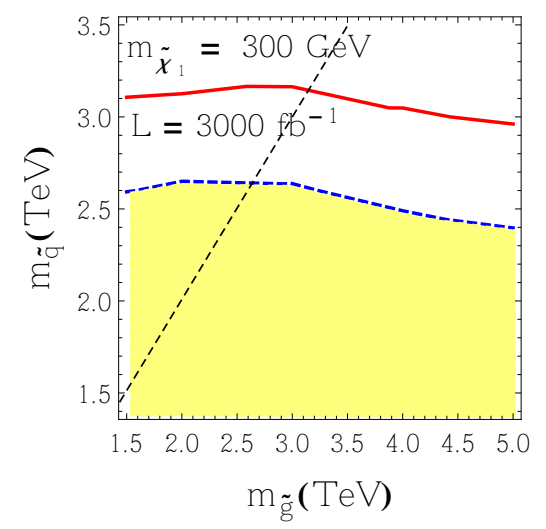

(d)

Figura 4.4: Regiões em destaque referem-se às massas que possuem potencial para descoberta e discernimento de supersimetria para determinados cenários. Foram empregados o conjunto Cortes Ótimos e Todos os sistemáticos com $\epsilon_{\text {taxa }}=20 \%$.

Podemos observar que na Fig. 4.4a e 4.4b, cenários de $100 \mathrm{fb}^{-1}$, onde estima-se alcança-los em meados de 2016 a 2017, têm regiões de descoberta-discernimento para massas de: $1.4 \mathrm{TeV}<$ $m_{\tilde{q}}<2.2 \mathrm{TeV}$ e $1.5 \mathrm{TeV}<m_{\tilde{g}}<4.7 \mathrm{TeV}$ com $m_{\tilde{\chi}_{1}}=m_{B^{(1)}}=50 \mathrm{GeV}$. Notamos um encolhimento da região de descoberta-discernimento quando a massa da matéria escura passa para 300 $\mathrm{GeV}$, essa observação fica mais clara com os resultados da seção anterior Fig. 4.3 . Agora as regiões de descoberta e possível discernimento de supersimetria e MUED, para $m_{\tilde{\chi}_{1}}=300 \mathrm{GeV}$, passam para $1.4 \mathrm{TeV}<m_{\tilde{q}}<2.1 \mathrm{TeV}$ e $1.4 \mathrm{TeV}<m_{\tilde{q}}<4.4 \mathrm{TeV}$. As regiões onde $m_{\tilde{g}}>3.5$ $\mathrm{TeV}$ e $m_{\tilde{q}} \sim 1.8 \mathrm{TeV}$, no caso de luminosidade integrada de $100 \mathrm{fb}^{-1}$, apresentam uma limitação 
para discernimento, veja a seção 4.1 .2 item 6

Já na Fig. $4.4 \mathrm{c}$ e $4.4 \mathrm{~d}$, vemos que as regiões de descoberta e discernimento não privilegiam aumento de massas em qualquer direção para $Z_{L L R}$. Nesse cenário a alta luminosidade compensa os efeitos de baixa estatística após cortes duros, porém algumas coisas não mudam, como o baixo alcance na massa dos squarks em comparação com a massa dos gluinos. Justamente porque na região que de $m_{\tilde{q}}<m_{\tilde{g}}$ temos a contribuição de um canal a mais para produção de jatos e MET. A região que pode ser testada com nosso trabalho para $L=3000 \mathrm{fb}^{-1}$ é de $1.4 \mathrm{TeV}<m_{\tilde{q}}<2.6 \mathrm{TeV}$ e de $1.4 \mathrm{TeV}<m_{\tilde{g}}<5.0 \mathrm{TeV}$.

Note que aumentar a luminosidade integrada em 30 vezes traz um aumento da região de exploração para descoberta e discernimento de aproximadamente $35 \%$. Esse é um resultado previsto para investigações de produção de partículas coloridas em altas energias [102]. A sensibilidade do LHC para alcançar massas cada vez maiores é limitada, estima-se que esse limite seja saturado por volta de $300 \mathrm{fb}^{-1}$. A partir de então, a coleta de dados será feita basicamente para tomar medidas de precisão do bóson de Higgs e de algum possível sinal de nova física na escala $\mathrm{TeV}$ que se manifeste antes desse limite de luminosidade. Por isso está planejado uma atualização para alcançar luminosidades de $3000 \mathrm{fb}^{-1}$ no LHC.

\subsection{Eventos de monte carlo para $m_{\tilde{\chi}_{1}}=1 \mathrm{TeV}$}

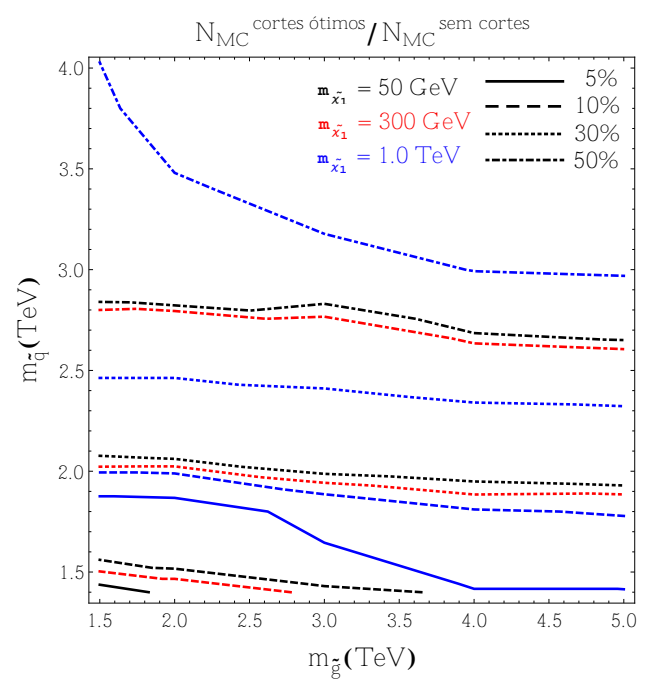

Figura 4.5: Curvas de níveis para frações de eventos de Monte Carlo depois e antes dos cortes. Região acima de uma dada curva tem maior fração de eventos do que a região abaixo da mesma curva.

O discernimento de modelos para neutralinos ou KK-fótons de 1 TeV não é possível usando a análise multivariada. Isso pode ser entendido através da Fig. 4.5, aqui avaliamos a fração de eventos de Monte Carlo $\left(f_{M C}\right)$ que passam pelo conjunto Cortes Ótimos para supersimetria. Ressaltamos que MUED tem comportamento parecido.

$$
f_{M C}=\frac{N_{M C}^{\text {cortes ótimos }}}{N_{M C}^{\text {sem cortes }}} .
$$

Por ser uma figura com curvas de níveis de valores diferentes, em contraste ao que estamos usando até aqui Fig. 4.1, devemos interpretá-la de maneira diferente. Agora regiões acima de uma determinada curva têm maior fração de eventos de Monte Carlo que passam pelo conjunto Cortes Ótimo, e regiões abaixo das curvas o contrário, por consequência. Podemos observar que, para um dado valor fixo de $f_{M C}$, as curvas em azul da Fig. 4.5, referentes à $m_{\tilde{\chi}_{1}}=1 \mathrm{TeV}$, sempre 
estão acima das curvas pretas e vermelhas, $m_{\tilde{\chi} 1}=50,300 \mathrm{GeV}$, respectivamente. Denotando que um neutralino pesado necessita de um espectro de squarks e gluinos mais pesados para fornecer a mesma fração $f_{M C}$ de eventos de Monte Carlo, após cortes duros, quando comparado com os espectros mais leves analisados nesse trabalho. Com poucos eventos após cortes retangulares, temos distribuições cinemáticas parecidas, e o discernimento de modelos para neutralinos de 1 $\mathrm{TeV}$ fica prejudicado.

\subsection{Formato das distribuições para diferentes $m_{\tilde{\chi}_{1}}$}

Podemos fazer uma observação interessante sobre a descoberta de supersimetria e discernimento de modelos, baseado no formato das distribuições. Veja a Fig. 4.6, que contém os nove observáveis físicos usados nesse trabalho, para dois casos de massas de neutralino (KK-fótons), de 50 e $300 \mathrm{GeV}$. As curvas sólidas representam o modelo de supersimetria, enquanto que as curvas pontilhadas o modelo de MUED, para massas de neutralino de 50 e $300 \mathrm{GeV}$. Note que, curvas sólidas de cores diferentes tem formato parecido, a mesma observação pode ser feita para as curvas pontilhadas, de MUED. Isso denota algo importante, no teste de hipóteses. Baseado na forma das distribuições cinemáticas, um modelo de supersimetria (MUED) com massas de neutralino com diferenças de alguns $\mathrm{GeV}$ têm formas parecidas, mostrando que, em um cenário de descoberta de supersimetria (MUED), a influência da massa desses neutralinos será pequena, ou seja, os resultados para as duas massas serão parecidos. Em contrapartida, se compararmos as curvas contínuas com as curvas pontilhadas, vemos que em uma análise de discernimento entre esses dois modelos, exitem diferenças significativas com relação à massa dos neutralinos, ou seja, os resultados para $m_{\tilde{\chi}_{1}}=50$ e $300 \mathrm{GeV}$, na discriminação de modelo, serão bem diferentes. Comportamento observado na descoberta e discernimento de modelos de nova física 4.2 e 4.3 .
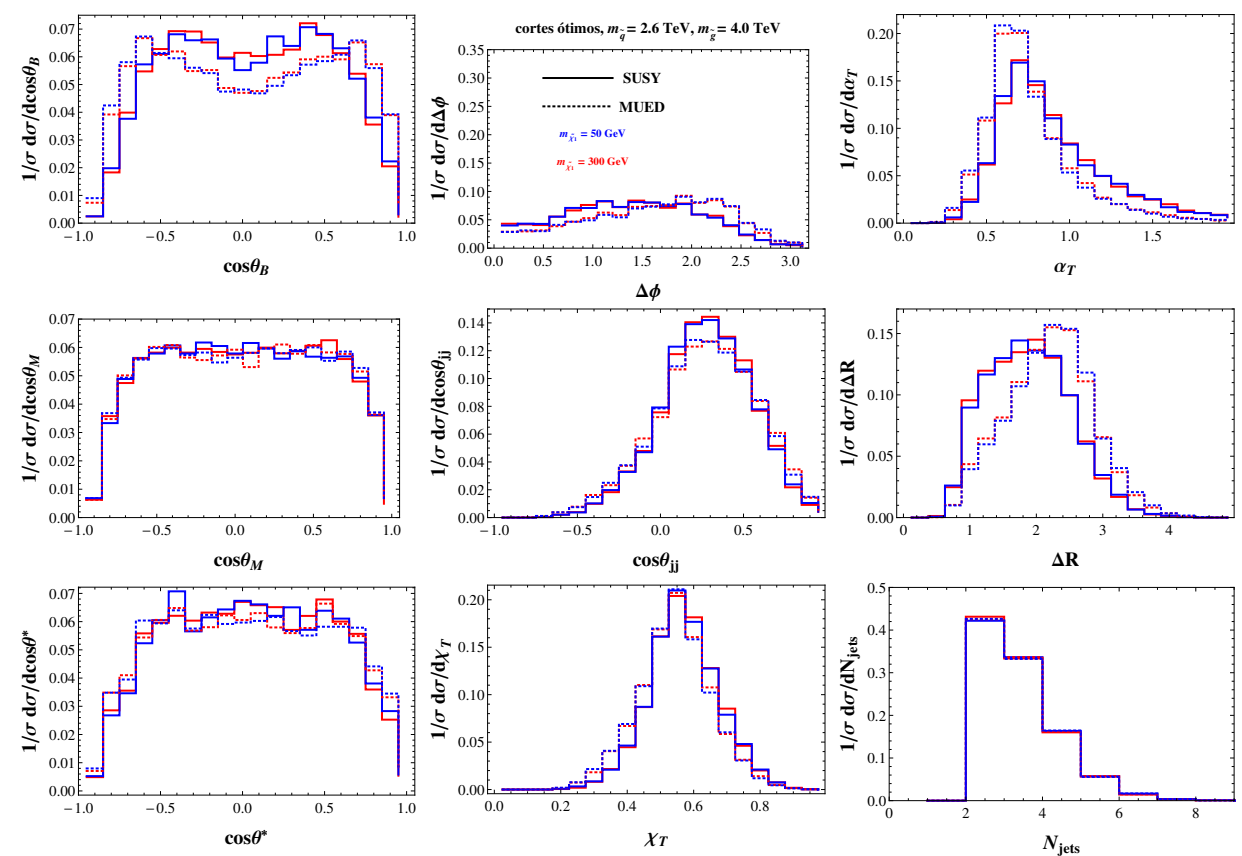

Figura 4.6: Dependência da forma da distribuições para supersimetria e MUED em dois cenários de massa de neutralinos (KK-fótons). 


\section{Capítulo 5}

\section{Conclusão}

A análise multivariada log-likelihood ratio utilizada em nosso trabalho mostra-se diferenciada, pois seu poder de descoberta de nova física é melhor do que a contagem de eventos. Isso porque na análise multivariada é possível combinar diversas informações contidas nas distribuições cinemáticas. Em média, o ganho de significância quando apenas um observável físico é utilizado na análise multivariada, é de $25 \%$. Constatamos também que o tagging de jatos de quarks e glúons potencializa a descoberta de supersimetria, mesmo em cenários com incertezas sistemáticas no sinal e backgrounds, nesse caso o ganho de significância é de quase 100\%. E ainda, na análise multivariada, a aplicação de incertezas sistemáticas pode ser feita de uma maneira direta através da marginalização do likelihood com a devida escolha de priors. As incertezas sistemáticas na taxa e formato da distribuições impõe restrições para a descoberta e discernimento de nova física. Apesar de alguma vezes negligenciada em trabalhos fenomenológicos, essas incertezas podem inviabilizar o alcance de muitas conclusões sobre processos envolvendo produção de partículas em colisores.

Em nosso caso, onde analisamos a produção de jatos e MET no LHC com $\sqrt{s}=14 \mathrm{TeV}$, partículas com carga de cor da QCD no estado final dificultam a descoberta e discernimento de modelos, devido basicamente a: grande topologia de diagramas envolvidos, os grandes backgrounds da QCD, as incertezas sistemáticas na taxa e formato das distribuições cinemáticas, a aplicação de cortes retangulares muito duros, o parton shower e a hadronização de partons. Todos esses processos influenciam fortemente a potencial correlação de novas partículas com os jatos produzidos de seus decaimentos. Além disso, não usamos modelos aproximados para supersimetria e MUED, onde os gluinos e KK-glúons estão desacoplados.

Nosso trabalho analisou cenários para squarks e gluinos de até $5.0 \mathrm{TeV}$. Nos limites para a massa do gluino vimos que nosso teste possui alcance maior do que $5.0 \mathrm{TeV}$, assim o espaço de massa verificado acabou limitando as massas de gluinos dentro das regiões de $5 \sigma$. É bem provável que a análise tenha um alcance maior do que 5.0 TeV para gluinos.

Em cenários com baixa incerteza sistemática na taxa dos eventos de background ( $\left.\epsilon_{\text {taxa }}\right)$, menor do que $10 \%$, o alcance de descoberta de um modelo simplificado de supersimetria para squarks é de até $2.95 \mathrm{TeV}$ com gluinos de até $5.0 \mathrm{TeV}$, para neutralinos de 50 a $300 \mathrm{GeV}$ com luminosidade integrada de $100 \mathrm{fb}^{-1}$. Aumentar a luminosidade para $3000 \mathrm{fb}^{-1}$, com esse nível de incerteza sistemática, traz um ganho para o alcance de squarks de $13 \%$. Com $\epsilon_{\text {taxa }}=20 \%$, temos uma diminuição do alcance das massas dos squarks de, em média, $5 \%$ para $100 \mathrm{fb}^{-1}$, e $7 \%$ para $3000 \mathrm{fb}^{-1}$. Cenários com $m_{\tilde{\chi}_{1}}=1 \mathrm{TeV}$, mostram-se acessíveis para descoberta de supersimetria, com $\epsilon_{\text {taxa }}=10 \%$ temos acesso a esses eventos com squarks de até $2.9 \mathrm{TeV}$, porém para $m_{\tilde{q}} \sim 1.4$ e $m_{\tilde{g}}>4.0 \mathrm{TeV}$ perdemos a capacidade de descobrir supersimetria com $5 \sigma$, com um neutralino pesado. Ressaltamos que uma procura de supersimetria com neutralinos de $1 \mathrm{TeV}$ possui poucos limites estabelecidos nos dias de hoje. A descoberta de supersimetria não se altera significativamente se o neutralino possuir massa de 50 ou $300 \mathrm{GeV}$, porém, no discernimento de modelos, essa diferença de massas influencia na capacidade de discernir supersimetria de MUED. 
O discernimento de modelos, para erros sistemáticos na taxa de eventos de backgrounds de $20 \%$, alcançam $2.15 \mathrm{TeV}$ para massa dos squarks e $4.3 \mathrm{TeV}$ para a massa gluinos com $m_{\tilde{\chi}_{1}}=$ $m_{B^{(1)}}=0.3 \mathrm{TeV}$, quando a luminosidade integrada é $100 \mathrm{fb}^{-1}$. Aumentando a luminosidade para $3000 \mathrm{fb}^{-1}$, temos um ganho de $\sim 25 \%$ para $m_{\tilde{q}}$ e de no mínimo $17 \%$ para $m_{\tilde{g}}$. Diminuindo a massa da matéria escura para $50 \mathrm{GeV}$ praticamente não altera o alcance de $m_{\tilde{q}}$, enquanto que $m_{\tilde{g}}$ aumenta em média de $30 \%$ para $100 \mathrm{fb}^{-1}$. Controlar a incerteza sistemática $\epsilon_{\text {taxa }}$ a níveis de $10 \%$, no discernimento de modelos, leva a um ganho médio de $50 \mathrm{GeV}$ para $m_{\tilde{q}}$ e de 100 $\mathrm{GeV}$ para $m_{\tilde{g}}$, quando comparado com o caso de $\epsilon_{\text {taxa }}=20 \%$. Para $m_{\tilde{\chi}_{1}}=m_{B^{(1)}}=1 \mathrm{TeV}$, o discernimento de SUSY versus MUED acaba sendo possível somente nos fictícios cenários de ausência de erros sistemáticos na taxa dos backgrounds. O discernimento de nova física, como um todo, ocorre em regiões do plano de massas de menor área quando comparado à descoberta de supersimetria. Isso denota que, para uma nova física do tipo supersimetria, poderemos dizer, inequivocamente, que temos um excesso com relação ao Modelo Padrão muito antes de poder confrontar supersimetria com MUED. Dependendo das incertezas sistemáticas, somente colisores do futuro poderão discernir possíveis modelos de nova física.

Nosso trabalho fez uso de técnicas já empregadas nos estudos de dados de nova física no LHC, junto à quantidade de trabalho computacional e concatenação de resultados que realizamos, através de modelos simplificados definidos como teorias efetivas na escala de energia do LHC, promovem nosso trabalho a uma análise sólida e pertinente na determinação dos limites desse colisor. Alcançamos resultados interessantes e outros trabalhos podem ser explorados com o que realizamos aqui. Pode-se testar hipóteses onde confronta-se modelos supersimétricos com paridade-R manifesta e parcialmente quebrada. Ou modelos de diferentes spin para matéria escura. Nossa análise mostra-se relevante não somente para análises de jatos e MET. 


\section{Apêndice A}

\section{Obtenção das Lagrangianas}

\section{A.1 Notação}

A métrica de $\eta$ é definida como $\eta^{\mu \nu}=(+,-,-,-)$.

As matrizes $\gamma$ em 4 dimensões são dadas por,

$$
\gamma^{5}=\left[\begin{array}{cc}
-1 & 0 \\
0 & 1
\end{array}\right], \gamma^{0}=\left[\begin{array}{ll}
0 & 1 \\
1 & 0
\end{array}\right], \gamma^{i}=\left[\begin{array}{cc}
0 & \sigma_{i} \\
-\sigma_{i} & 0
\end{array}\right]
$$

Um espinor em de quatro componentes pode ser dado por,

$$
\psi=\left[\begin{array}{l}
\psi_{L} \\
\psi_{R}
\end{array}\right]
$$

Onde,

$$
P_{R, L} \psi=\psi_{R, L}=\frac{1}{2}\left(1 \pm \gamma_{5}\right)\left[\begin{array}{l}
\psi_{L} \\
\psi_{R}
\end{array}\right] .
$$

As matrizes $\gamma^{\mu} 4 x 4$ podem ser, eventualmente, representadas por matrizes $2 x 2$ de Pauli $\sigma^{1,2,3} \equiv \vec{\sigma}$

$$
\gamma^{\mu}=\left[\begin{array}{cc}
0 & \sigma^{\mu} \\
\bar{\sigma}^{\mu} & 0
\end{array}\right]
$$

Onde $\sigma^{\mu}=(1, \vec{\sigma})$ e $\bar{\sigma}^{\mu}=(1,-\vec{\sigma})$ De forma mais geral temos:

$$
\bar{\sigma}^{\mu \dot{\alpha} \alpha}=\epsilon^{\dot{\alpha} \dot{\beta}} \epsilon^{\alpha \beta} \sigma_{\beta \dot{\beta}}^{\mu} .
$$

Espinores de Majorana serão dados por,

$$
\Psi_{M}=\left[\begin{array}{c}
\psi \\
\psi^{\dagger}
\end{array}\right]
$$

A componente $\psi$ possui índices espinoriais que possuem uma métrica não diagonal, dada por:

$$
\epsilon^{\alpha \beta}=-\epsilon_{\alpha \beta}=\left[\begin{array}{cc}
0 & 1 \\
-1 & 0
\end{array}\right]
$$

De modo que $\psi_{\alpha}=\epsilon_{\alpha \beta} \psi^{\beta}$. Todas as componentes espinorias são grassmanianos, ou seja, $\left\{\psi_{\alpha}, \psi_{\beta}\right\}=0$. Uma relação equivalente pode ser estipulada para os índices com pontos $\psi_{\dot{\alpha}}=$ $\epsilon_{\dot{\alpha} \dot{\beta}} \psi^{\dot{\beta}}$, esse índices transformam-se sob uma representação complexa do grupo de Lorentz com relação aos índices sem pontos. Pode-se definir $\psi_{\alpha}^{\dagger}=\psi_{\dot{a}}^{*}$.

\footnotetext{
${ }^{1}$ Os índices espinoriais para essa relação devem ser $\left(\sigma^{\mu}\right)_{\alpha \dot{\alpha}}$ e $\left(\bar{\sigma}^{\mu}\right)^{\dot{\alpha} \alpha}$.

${ }^{2} \mathrm{O}$ complexo conjugado pode ser escrito assim pois os campos desses espinores anti-comutantes são considerados clássicos.
} 
Os índices $M, N$ são reservados para análises em 5 dimensões, onde a dimensão extra é fechada. As matrizes $\Gamma$ tornam-se: $\Gamma^{M}=\left(\gamma^{\mu}, i \gamma^{5}\right)$. A métrica torna-se:

$$
g^{M N}=\left[\begin{array}{cc}
\eta^{\mu \nu} & 0 \\
0 & -1
\end{array}\right]
$$

As matrizes de Gell-Mann para a QCD $\left(S U(3)_{C}\right)$ são dadas pelas 8 matrizes $\lambda^{a}$. Essas matrizes satisfazem a relação de comutação:

$$
\left[\lambda^{a}, \lambda^{b}\right]=2 i f^{a b c} \lambda^{c}
$$

$f^{a b c}$ é totalmente anti-simétrico em seus índices. Definiremos $T^{a}=\frac{\lambda^{a}}{2}$ nesse trabalho.

As lagrangianas serão explicitadas com índices de cor $S U(3)$ suprimidos, nas exceções diremos previamente a mudança. Os estados gauge (dubleto $\left.S U(2)_{L}\right)$ serão denotados por:

$$
Q=\left[\begin{array}{l}
Q^{u} \\
Q^{d}
\end{array}\right]
$$

Os estados físicos por:

$$
q=\left[\begin{array}{c}
q_{L} \\
q_{R}^{\dagger}
\end{array}\right]
$$

E os parceiros supersimétricos por $\tilde{q}_{L}$ e $\tilde{q}_{R}$.

Para os campos 5 dimensionais é comum definí-los como funções trigonométricas periódicas na dimensão extra. As relações de ortogonalidade de funções trigonométricas utilizadas nesse trabalho foram:

$$
\begin{array}{r}
\frac{1}{2} \int_{-\pi R}^{\pi R} d y \cos \left(\frac{m y}{R}\right) \cos \left(\frac{n y}{R}\right)=\frac{\pi R \delta_{m}}{2}, \\
\frac{1}{2} \int_{-\pi R}^{\pi R} d y \sin \left(\frac{m y}{R}\right) \sin \left(\frac{n y}{R}\right)=\frac{\pi R \delta_{m n}}{2}, \\
\frac{1}{2} \int_{-\pi R}^{\pi R} d y \cos \left(\frac{m y}{R}\right) \sin \left(\frac{n y}{R}\right)=0, \\
\frac{1}{2} \int_{-\pi R}^{\pi R} d y \cos \left(\frac{m y}{R}\right) \cos \left(\frac{n y}{R}\right) \cos \left(\frac{l y}{R}\right)=\frac{\pi R \Delta_{m n l}^{1},}{4}, \\
\frac{1}{2} \int_{-\pi R}^{\pi R} d y \sin \left(\frac{m y}{R}\right) \sin \left(\frac{n y}{R}\right) \cos \left(\frac{l y}{R}\right)=\frac{\pi R \Delta_{m n l}^{4},}{4}, \\
\frac{1}{2} \int_{-\pi R}^{\pi R} d y \sin \left(\frac{m y}{R}\right) \sin \left(\frac{n y}{R}\right) \sin \left(\frac{l y}{R}\right)=0, \\
\frac{1}{2} \int_{-\pi R}^{\pi R} d y \sin \left(\frac{m y}{R}\right) \cos \left(\frac{n y}{R}\right) \cos \left(\frac{l y}{R}\right)=0 .
\end{array}
$$

Onde,

$$
\begin{gathered}
\Delta_{m n l}^{1}=\delta_{l, m+n}+\delta_{n, m+l}+\delta_{m, l+n}, \\
\Delta_{m n l}^{4}=-\delta_{l, m+n}+\delta_{n, m+l}+\delta_{m, l+n} .
\end{gathered}
$$

A densidade lagrangiana será sempre denotada por $\mathcal{L}$, de modo que a lagrangiana total é dada por $L=\int d x^{4} \mathcal{L}$. 


\section{A.2 Supersimetria}

A álgebra supersimétrica em 4 dimensões $\left(x^{\mu}=(t, \vec{x})\right)$ é dada por geradores fermiônicos que satisfazem as seguintes condições:

$$
\begin{gathered}
\left\{Q_{\alpha}, Q_{\dot{\alpha}}^{\dagger}\right\}=2 \sigma_{\alpha \dot{\alpha}}^{\mu} P_{\mu}, \quad\left\{Q_{\alpha}, Q_{\alpha}\right\}=\left\{Q_{\dot{\alpha}}^{\dagger}, Q_{\dot{\alpha}}^{\dagger}\right\}=0, \\
{\left[P^{\mu}, Q_{\alpha}\right]=\left[P^{\mu}, Q_{\dot{\alpha}}^{\dagger}\right]=0 .}
\end{gathered}
$$

Onde $P$ é o momento no espaço-tempo quadridimensional. Os operadores $Q$ e $Q^{\dagger}$ são os geradores de supersimetria e transformam-se sob boosts e translações como objetos de spin- $1 / 2$.

Os geradores $Q$ e $Q^{\dagger}$ podem ser escritos em teoria quântica de campos como correntes conservadas. Aproveitando o fato de que o momento $P^{\mu}$ comuta com esses geradores, podemos construir representações irredutíveis de supersimetria.

As representações de supersimetria são dadas por um conjunto de estados com mesma massa $m\left(P^{2}=m^{2}\right)$. Além disso temos o mesmo número de graus de liberdade bosônicos e fermiônicos, para uma dada representação. O conjunto de partículas que compõe uma dada representação pode ser agrupado em um supercampo. Esses campos são explicitados em um superespaço $\left(x^{\mu}, \theta, \theta^{\dagger}\right)$. Na linguagem de supercampos as transformações de supersimetria tornam-se uma espécie de transformações de gauge com parâmetros grassmanianos $\left(\epsilon, \epsilon^{\dagger}\right)$ e as lagrangianas podem ser escritas com a invariância supersimétrica explícita, chamamos de lagrangianas com supersimetria manifesta.

As transformações supersimétricas $\delta_{\xi}$, onde $\xi$ é um parâmetros grassmaniano, podem ser escritas como:

$$
\delta_{\xi} F\left(x, \theta, \theta^{\dagger}\right)=\left(\xi Q+\xi^{\dagger} Q^{\dagger}\right) F\left(x, \theta, \theta^{\dagger}\right) .
$$

Onde $F$ pode ser um supercampo quiral $\Phi$ :

$$
\Phi\left(x^{\mu}\right) \equiv\{\phi(x), \psi(x), F(x)\} .
$$

Esse supermultipleto é composto por um campo escalar complexo $\phi(x)$, um campo espinorial de duas componentes $\psi(x)$ e um campo auxiliar ${ }^{3}$ complexo $F(x)$.

$F$ também pode ser uma supercampo vetorial ou real $V=V^{\dagger}$, nesse caso o supermultipleto contém campos vetoriais, que também possui invariância gauge. No gauge de Wess-Zumino o campo vetorial torna-se:

$$
V\left(x^{\mu}\right) \equiv\left\{A^{\mu}, \lambda, \lambda^{\dagger}, D\right\}
$$

Onde $A^{\mu}$ é um campo vetorial, $\lambda$ e $\lambda^{\dagger}$ são as componentes de um espinor de majorana e $D$ um campo escalar real. O gauge de Wess-Zumino pode ser definido em teorias gauge não-abelianas.

Para termos supersimetria manifesta em uma lagrangiana com um campo escalar e seu parceiro supersimétrico precisamos das seguintes transformações supersimétricas:

$$
\begin{aligned}
\delta_{\xi} \phi & =\sqrt{2} \xi \psi, \\
\delta_{\xi} \psi & =i \sqrt{2} \sigma^{\mu} \xi^{\dagger} \partial_{\mu} \phi+\sqrt{2} \xi F, \\
\delta_{\xi} F & =i \sqrt{2} \xi^{\dagger} \bar{\sigma}^{\mu} \partial_{\mu} \psi .
\end{aligned}
$$

Onde a lagrangiana invariante sob esse conjunto de transformações $\left(\delta_{\xi} \mathcal{L}\right)$ é:

$$
\mathcal{L}_{\text {quiral }}=i \psi^{\dagger} \sigma^{\mu} \partial_{\mu} \psi+\phi^{*} \partial^{2} \phi+F^{*} F .
$$

\footnotetext{
${ }^{3}$ Campos auxiliares são todos aqueles que não têm um termo cinético na lagrangiana.
} 
Aqui $\phi$ é um campo escalar complexo, $\psi$ um espinor de Weyl de duas componentes e $F$ um campo auxiliar, escalar e complexo. O campo auxiliar é introduzido para tornar supersimetria uma simetria fora da camada de massa. Caso despreze-se o campo auxiliar $F$, perde-se a igualdade de graus de liberdade bosônicos e fermiônicos, assim as transformações de supersimetria só serão uma simetria da lagrangiana $\mathcal{L}_{\text {quiral }}$ se usarmos as equações de movimento para $\phi$ e $\psi$, chamamos esse tipo de simetria de supersimetria na camada de massa.

A lagrangiana para os glúons $\left(g_{\mu}^{a}\right)$ e seu parceiro supersimétrico, o gluino $\left(\tilde{g}^{a}\right.$ em notação de 4 componentes ou $\lambda, \lambda^{\dagger}$ para a notação em 2 componentes), é escrita na representação adjunta de $S U(3)_{C}$. No gauge de Wess-Zumino é necessário a introdução de um campo auxiliar escalar real $\left(D^{a}\right)$ também na representação adjunta de $S U(3)_{C}$ para as transformações de supersimetria tornarem-se uma simetria da lagrangiana sem equações de movimento.

$$
\mathcal{L}_{\text {gauge }}=-\frac{1}{4} G^{a \mu \nu} G_{\mu \nu}^{a}+i \lambda^{\dagger a} \bar{\sigma}^{\mu} \mathcal{D}_{\mu} \lambda^{a}+\frac{1}{2} D^{a} D^{a} .
$$

Onde $G_{\mu \nu}^{a}=\partial_{\mu} g_{\nu}^{a}-\partial_{\nu} g_{\mu}^{a}+g f^{a b c} g_{\mu}^{b} g_{\nu}^{c}$

As derivadas covariantes para campos na representação fundamental $(\phi)$ e adjunta $\left(\phi^{a}\right)$ são dadas por:

$$
\begin{aligned}
\mathcal{D}_{\mu} \phi & =\partial_{\mu} \phi-i g_{s} g_{\mu} \phi, \\
\mathcal{D}_{\mu} \phi^{a} & =\partial_{\mu} \phi^{a}+g_{s} f^{a b c} g_{\mu}^{b} \phi^{c} .
\end{aligned}
$$

Onde $g_{\mu}=g_{\mu}^{a} T^{a}$. Onde $T^{a}$ são as matrizes de Gell-Mann A.1 a menos de um fator $\frac{1}{2}$.

As transformações supersimétricas para o glúon, gluino e campo auxiliar são:

$$
\begin{aligned}
\delta_{\xi} g^{a \mu} & =-i \lambda^{a \dagger} \bar{\sigma}^{\mu} \xi+i \xi^{\dagger} \bar{\sigma}^{\mu} \lambda^{a}, \\
\delta_{\xi} \lambda^{a} & =\sigma^{\mu \nu} \xi g_{\mu \nu}^{a}+i \xi D^{a}, \\
\delta_{\xi} D^{a} & =-\xi \sigma^{\mu} \mathcal{D}_{\mu} \lambda^{\dagger a}-\mathcal{D}_{\mu} \lambda^{a} \sigma^{\mu} \xi^{\dagger} .
\end{aligned}
$$

Enquanto que as transformações gauge, com parâmetro infinitesimal $\Theta$, são:

$$
\begin{aligned}
\delta_{\text {gauge }} g_{\mu}^{a} & =\partial_{\mu} \Theta^{a}+g_{s} f^{a b c} g_{\mu}^{b} \Theta_{\nu}^{c}, \\
\delta_{\text {gauge }} \lambda^{a} & =g_{s} f^{a b c} \lambda^{b} \Theta^{c} \\
\delta_{\text {gauge }} D^{a} & =g_{s} f^{a b c} D^{b} \Theta^{c} .
\end{aligned}
$$

A introdução de interações entre os campos de gauge $S U(3)_{C}$, os glúons, e a matéria, os quarks, pode ser feita de maneira supersimétrica e invariante de gauge. Para uma descrição completa da QCD temos que ter os campos de matéria na representação fundamental do grupo de gauge, ou seja, os quarks, os squarks e os campos auxiliares. Porém as componentes Left e Right dos quarks têm números quânticos gauge diferentes, e por isso deve-se inclú́-los em supermultipletos separados. Isso implica na introdução de dois squarks diferentes, $\tilde{q}_{R}$ e $\tilde{q}_{L}$, como parceiros supersimétricos do spinor de Dirac para o quark $q=\left(\begin{array}{ll}q_{L} & q_{R}\end{array}\right)$. A lagrangiana de interação é:

$$
\mathcal{L}_{\text {interação }}=-\sqrt{2} g_{s}\left(\tilde{q}_{i}^{*} T^{a} q_{i}\right) \lambda^{a}-\sqrt{2} g_{s} \bar{\lambda}^{a}\left(\bar{q}_{i} T^{a} \tilde{q}_{i}\right)+g_{s}\left(\tilde{q}^{*} T^{a} \tilde{q}\right) D^{a} .
$$

Uma das notáveis características de supersimetria é que a interação dos squarks, glúons e algumas das componentes dos gluinos ocorrem com o mesmo acoplamento $g_{s}$. Esse fato propicia o cancelamento de divergências quadráticas na massa do bóson de Higgs para o MSSM.

A lagrangiana total:

$$
\mathcal{L}_{\text {susy-QCD }}=\mathcal{L}_{\text {quiral }}+\mathcal{L}_{\text {gauge }}+\mathcal{L}_{\text {interação }}
$$


Onde $\mathcal{L}_{\text {quiral }}$ e $\mathcal{L}_{\text {gauge }}$ são construídas através das substituições:

$$
\begin{aligned}
\partial_{\mu} \tilde{q}_{i} & \rightarrow \mathcal{D}_{\mu} \tilde{q}_{i}=\partial_{\mu} \tilde{q}_{i}-i g_{s} g_{\mu}^{a} T^{a} \tilde{q}_{i} \\
\partial_{\mu} q_{i} & \rightarrow \mathcal{D}_{\mu} q_{i}=\partial_{\mu} q_{i}-i g_{s} g_{\mu}^{a} T^{a} q_{i}
\end{aligned}
$$

Todos os índices de cores estão subentendidos e os índices $i$ referem-se às quiralidades, Left e Right.

$\mathcal{L}_{\text {susy-QCD }}$ invariante sob as transformações supersimétricas:

$$
\begin{aligned}
\delta_{\xi} \tilde{q}_{i} & =\xi q_{i}, \\
\delta_{\xi} q_{i} & =-i \sigma^{\mu} \bar{\xi} \mathcal{D}_{\mu} \tilde{q}_{i}+\xi F_{i} \\
\delta_{\xi} F_{i} & =-i \bar{\xi} \bar{\sigma}^{\mu} \mathcal{D}_{\mu} q_{i}+\sqrt{2} g_{s} T^{a} \tilde{q}_{i} \bar{\xi} \overline{\tilde{g}}^{a} .
\end{aligned}
$$

Temos condições agora de deduzir a lagrangina usada para criar o modelo de supersimetria usado na produção de jatos e MET com decaimento direto de squarks em jatos e neutralino (2.9).

- O termo $\mathcal{L}_{q \tilde{q} \tilde{q}}$ vem da derivada covariante para os squarks A.28 e do termo cinético escalar de A.16. É interessante rearranjar o termo cinético de $\tilde{q}^{*} \partial^{2} \tilde{q}$ para $-\partial_{\mu} \tilde{q}^{*} \partial^{\mu} \tilde{q}+$ (derivadas totais). Usamos $\tilde{q}^{*} \overleftrightarrow{\partial_{\mu}} \tilde{q}=\left(\partial_{\mu} \tilde{q}^{*}\right) \tilde{q}-\tilde{q}^{*}\left(\partial_{\mu} \tilde{q}\right)$. Então obtemos,

$$
\mathcal{L}_{g \tilde{q} \tilde{q}}=-i g_{s} \sum_{q}\left[\tilde{q}_{L}^{*} \overleftrightarrow{\partial_{\mu}} \tilde{q}_{L}+\tilde{q}_{R}^{*} \overleftrightarrow{\partial_{\mu}} \tilde{q}_{R}\right] T^{a} g^{a \mu}
$$

- O termo $\mathcal{L}_{g \tilde{g} \tilde{g}}$ virá da derivada covariante A.28 para o gluino da lagrangiana A.17. Porém a lagrangiana está escrita em termos de espinores de duas componentes, para escrevermos na forma de espinores de 4 componentes precisamos definir o spinor de majorana para o gluino da seguinte forma,

$$
\tilde{g}^{a}=\left[\begin{array}{c}
-i \lambda^{a} \\
i \bar{\lambda}^{a}
\end{array}\right]
$$

O resultado será 2.11,

$$
\mathcal{L}_{g \tilde{g} \tilde{g}}=i \frac{1}{2} g_{s} f^{a b c} \overline{\tilde{g}}^{a} \gamma_{\mu} \tilde{g}^{b} g^{c \mu}
$$

- Para $\mathcal{L}_{g g \tilde{q} \tilde{q}}$ devemos recuperar o termo cinético de A.28 com a derivada covariante para os squarks, mantendo os termos que contém 2 glúons e dois squarks temos,

$$
\mathcal{L}_{g g \tilde{q} \tilde{q}}=g_{s}^{2} \sum_{q}\left[\tilde{q}_{L \mathbf{i}}^{*} \tilde{q}_{L \mathbf{j}}+\tilde{q}_{R \mathbf{i}}^{*} \tilde{q}_{R \mathbf{j}}\right]\left(T^{a} T^{b}\right)_{\mathbf{i j}} g^{a \mu} g_{\mu}^{b}
$$

Onde os índices em negrito $\mathbf{i}, \mathbf{j}=1,2,3$, referem-se à representação fundamental $S U(3)_{C}$, as cores dos quarks e squarks.

- O termo $\mathcal{L}_{q \tilde{q} \tilde{g}}$ virá da lagrangiana de interações A.26. Novamente teremos que colocar a lagrangiana em notação para espinores de 4 componentes, para isso usaremos novamente $\tilde{g}^{a}$ e o espinor de quatro componentes para os quarks A.5.

$$
\mathcal{L}_{q \tilde{q} \tilde{g}}=-\sqrt{2} g_{s} \sum_{q}\left[\bar{q} P_{R} T^{a} \tilde{g}^{a} \tilde{q}_{L}-\bar{q} P_{L} T^{a} \tilde{g}^{a} \tilde{q}_{R}\right]+\text { h.c.. }
$$

- O termo $\mathcal{L}_{q \tilde{q} \tilde{\chi}_{1}}$ depende do esquema de quebra soft de supersimetria, tabela 2.2 Porém estamos interessados em modelos onde o decaimento de squarks seja totalmente em quarks 
e uma partícula pesada, neutra e que interage fracamente $(g)$. Isso pode ser alcançado de uma forma geral com,

$$
\begin{array}{r}
\mathcal{L}_{q \tilde{q} \tilde{\chi}_{1}}=-\frac{g}{\sqrt{2}} \overline{\tilde{\chi}}_{1}^{0}\left[\left(l_{q}^{L} P_{L}+r_{q}^{L} P_{R}\right) \tilde{q}_{L}^{*}+\left(l_{q}^{R} P_{L}+r_{q}^{R} P_{R}\right) \tilde{q}_{R}^{*}\right] q \\
=+ \text { h.c. }
\end{array}
$$

Assumimos um valor inicial para esses parâmetros de modo a alcançar os valores de taxas e massas explicitados na tabela 3.2 .

Os vértices relevantes para produção de squarks e gluinos estão explicitados na Fig. A.1. Os diagramas de Feynman relacionados com a produção de squark no LHC e na produção de squarks e gluinos estão listados nas Fig. 3.2 e 3.3 .
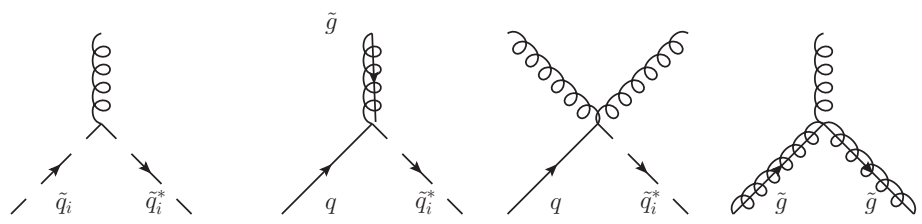

Figura A.1: Vértices envolvidos na produção de squarks e gluinos através de colisões prótonpróton.

\section{A.3 MUED}

Assumindo que todos os campos do Modelo Padrão podem se propagar pela dimensão extra compacta de raio $R$. Podemos observar que o momento $p^{5}$ é conservado e os campos obedecem condições periódicas na dimensão extra, ou seja $\phi(x, y)=\phi(x, y+2 \pi R)$. Com isso os campos podem ser expandidos em $\mathrm{D}=4$ como:

$$
\left.\phi(x, y)=\frac{1}{\sqrt{\pi R}} \sum_{n=0}^{\infty}\left[\phi^{(n)}(x) \cos \left(\frac{n \pi y}{R}\right)\right)+\hat{\phi}^{(n)}(x) \sin \left(\frac{n \pi y}{R}\right)\right]
$$

Onde $\phi^{(n)}(x)$ e $\phi^{(n)}(x)$ são as componentes em 4D de um campo $\phi(x, y)$. Ou de forma equivalente podemos explicitar o modo zero $n=0$ junto ,

$$
\begin{aligned}
\phi(x, y)= & \frac{1}{\sqrt{2 \pi R}} \phi_{0}(x)+\frac{1}{\sqrt{\pi R}} \sum_{n=1}^{\infty}\left[\phi^{(n)}(x) \cos \left(\frac{n \pi y}{R}\right)\right)+ \\
& \left.\hat{\phi}^{(n)}(x) \sin \left(\frac{n \pi y}{R}\right)\right] .
\end{aligned}
$$

A normalização do modo zero dever ser diferente, pois as integrações de seno e cosseno fornecem resultados diferentes da integração da dimensão extra somente.

$\mathrm{Em} \mathrm{D}=5$ não é possível definir matrizes que comutem entre si e cumpram $(A)^{2}=\mathbf{1}$ assim como feito para as matrizes $\gamma^{\mu}$ em A.1. Por isso trata-se os férmions em $\mathrm{D}=5$ como um espinor de 4 componentes, denotaremos por $\psi(x, y)$. Porém sabemos que férmions são quirais e interagem com o modelo eletro-fraco de maneira distinta. Para os férmions teremos uma expansão equivalente a A.40, porém com duas quiralidades $\psi_{L}$ e $\psi_{R}$.

Para os campos de gauge $A(x, y)$ também teremos uma expansão idêntica à A.40. Porém a característica vetorial desses bósons leva a um campo extra escalar em $\mathrm{D}=4$, o $A_{5}(x)\left(A_{M} \equiv\right.$ $\left.\left(A_{5}, A_{\mu}\right)\right)$.

Define-se uma paridade na quinta dimensão, $Z_{2}: y \rightarrow-y$, de modo que a dimensão extra reduz-se a $S_{1} / Z_{2}$ Fig. 2.6. Podemos observar que assumindo $\phi(x, y)$ par sob $Z_{2}$ (A.40, ou seja, 
$\phi(x, y) \rightarrow+\phi(x,-y)$. Então teremos modo zero. Caso contrário o modo zero deve deixar a expansão:

$$
\begin{aligned}
\phi^{+}(x, y) & =\frac{1}{\sqrt{2 \pi R}} \phi_{0}^{+}(x)+\frac{1}{\sqrt{\pi R}} \sum_{n=1}^{\infty} \phi^{+(n)}(x) \cos \left(\frac{n \pi y}{R}\right) \\
\phi^{-}(x, y) & =\frac{1}{\sqrt{\pi R}} \sum_{n=1}^{\infty} \phi^{-(n)}(x) \sin \left(\frac{n \pi y}{R}\right)
\end{aligned}
$$

Onde $\phi^{+}$indica um campo par sob $Z_{2}$ e $\phi^{-}$indica um campo ímpar sob $Z_{2}$.

O mesmo pode ser feito para os bósons de gauge (campos vetoriais em 5D). Se $A(x, y)$ for par sob $Z_{2}$ teremos modo zero para esse campo. Com isso deve-se ter $A_{5}(x, y)$ ímpar sob $Z_{2}$, para conservação da ação gauge, logo $A_{5}$ não terá modo zero. O que é uma grande vantagem, pois teríamos que inserir um escalar $A_{5}$ para cada bóson de gauge do Modelo Padrão. Assim temos,

$$
\begin{aligned}
& A_{\mu}(x, y)=\frac{1}{\sqrt{2 \pi R}} A_{\mu}^{0}(x)+\frac{1}{\sqrt{\pi R}} \sum_{n=1}^{\infty} A_{\mu}^{(n)}(x) \cos \left(\frac{n \pi y}{R}\right) \\
& A_{5}(x, y)=\frac{1}{\sqrt{\pi R}} \sum_{n=1}^{\infty} A_{5}^{(n)}(x) \sin \left(\frac{n \pi y}{R}\right) .
\end{aligned}
$$

Os KK-bósons de gauge $\left(A^{(n)}(x)\right)$ adquirem massa através dos bósons escalares $A_{5}^{(n)}(x)$, porém o modo zero de $A_{\mu}$ permanecem sem massa, pois $A_{5}$ não tem modo zero ${ }^{4}$. É possível ainda fixar um gauge usando invariância gauge em $\mathrm{D}=5\left(A_{M} \rightarrow A_{M}+\partial_{M} \Lambda(x, y)\right)$. Resultando em $A_{5}(x, y)=0$ enquanto que o bóson de gauge $A_{\mu}(x, y)$ permanece como o dado em A.43) e com massa $\frac{n}{R}$.

Para os férmions iremos assumir o seguinte:

$$
\begin{gathered}
\gamma_{5} \psi_{L}^{(n)}(x)=-\psi_{L}^{(n)}(x), \\
\gamma_{5} \psi_{R}^{(n)}(x)=+\psi_{R}^{(n)}(x) .
\end{gathered}
$$

Assim podemos definir a operação $Z_{2}$ sobre espinor $\psi=\left(\psi_{L} \quad \psi_{R}\right)$ como $\gamma_{5} \psi(x, y)= \pm \psi(x, y)$. Explicitamente teremos,

$$
\begin{aligned}
& \psi^{+}(x, y)=\frac{1}{\sqrt{2 \pi R}} \psi_{R}^{0}(x)+\frac{1}{\sqrt{\pi R}} \sum_{n=1}^{\infty}\left[\psi_{R}^{(n)}(x) \cos \left(\frac{n \pi y}{R}\right)+\psi_{L}^{(n)}(x) \sin \left(\frac{n \pi y}{R}\right)\right] \\
& \psi^{-}(x, y)=\frac{1}{\sqrt{2 \pi R}} \psi_{L}^{0}(x)+\frac{1}{\sqrt{\pi R}} \sum_{n=1}^{\infty}\left[\psi_{L}^{(n)}(x) \cos \left(\frac{n \pi y}{R}\right)+\psi_{R}^{(n)}(x) \sin \left(\frac{n \pi y}{R}\right)\right] .
\end{aligned}
$$

Note que no caso do Modelo Padrão em dimensões extras universais teremos, por exemplo, um dubleto de quarks $Q_{L}=\left(\begin{array}{ll}u_{L} & d_{L}\end{array}\right)$ junto com KK-quarks Left $\left(u_{L}^{(n)}\right)$ e Right $\left(u_{R}^{(n)}\right)$, o mesmo para os quarks $d$. Os KK-quarks terão uma interação vetorial com os bósons e KK-bósons de gauge. O mesmo para os singletos $S U(2)$ do Modelo Padrão $u_{R}$ e $d_{R}$, terão uma torre KK com partículas Left e Right, que interagem igualmente com os bósons e KK-bósons de gauge.

A dimensão de massa para os campos fermiônico e bosônico gauge em $\mathrm{D}=5$ é 2 e $\frac{3}{2}$, o que leva a acoplamentos em $\mathrm{D}=5 \mathrm{com}$ dimensão de massa negativos. Causando a não-renormalização de MUED. Porém MUED é tratada como uma teoria efetiva, de validade até uma dada escala $\Lambda_{\text {MUED }}$. Essa escala é um dos parâmetros livres para o modelo, assim como o raio de compatificação $R$.

\footnotetext{
${ }^{4} \mathrm{O}$ ganho de massa para os bósons de gauge vem do fato de assumir paridades específicas para esses campos, como feito em A.43.
} 
Os campos para MUED relevantes para produção de jatos e MET analisados em nosso trabalho são os quarks e bósons de gauge. Iremos manter apenas os primeiros estados excitados, pois esses estados possuem análogos em supersimetria com spins diferentes. Começando pelos bósons de gauge temos:

$$
\begin{aligned}
B_{\mu}(x, y) & =\frac{1}{\sqrt{\pi R}}\left[B_{\mu}^{0}(x)+\sqrt{2} B_{\mu}^{(1)}(x) \cos \frac{y}{R}\right], \\
G_{\mu}(x, y) & =\frac{1}{\sqrt{\pi R}}\left[G_{\mu}^{0}(x)+\sqrt{2} G_{\mu}^{(1)}(x) \cos \frac{y}{R}\right] .
\end{aligned}
$$

Por questão de adequação com os programas usados para simulação de eventos explicitamos os campos com uma normalização diferente. A dimensão extra agora estende-se de $-\pi R$ a $+\pi R$. $B^{\mu}$ é o fóton pesado e $G^{\mu}$ o glúon em 5 dimensões. Todas as componentes da quinta dimensão desses campos foram retiradas por uma transformação de gauge. O bóson de gauge para o glúon em $\mathrm{D}=5$, é dado por $G_{M}=G_{M}^{a} \frac{\lambda^{a}}{2}$, onde $a$ são índices da representação adjunta de $S U(3)_{C}$.

Os campos de massa serão os quarks. Denotaremos dubletos $S U(2)_{L}$ por $Q_{L}$ e os singletos $U(1)_{Y}$ por $q_{R}$, onde $q$ denotam quarks $u, d, c, s$. Serão dados por:

$$
\begin{aligned}
Q(x, y) & =\frac{1}{\sqrt{\pi R}}\left[q_{L}(x)+\sqrt{2} P_{L} Q_{L}^{(1)}(x) \cos \frac{y}{R}+\sqrt{2} P_{R} Q_{R}^{(1)}(x) \sin \frac{y}{R}\right], \\
U(x, y) & =\frac{1}{\sqrt{\pi R}}\left[u_{R}(x)+\sqrt{2} P_{L} u_{L}^{(1)}(x) \sin \frac{y}{R}+\sqrt{2} P_{R} u_{R}^{(1)}(x) \cos \frac{y}{R}\right], \\
D(x, y) & =\frac{1}{\sqrt{\pi R}}\left[d_{R}(x)+\sqrt{2} P_{L} d_{L}^{(1)}(x) \sin \frac{y}{R}+\sqrt{2} P_{R} d_{R}^{(1)}(x) \cos \frac{y}{R}\right] .
\end{aligned}
$$

Além do conteúdo do Modelo Padrão $Q, u$ e $d$, deve-se introduzir um conjunto de estados excitados todos com interações do tipo vetorial. Os dubletos $S U(2)_{L}$ são definidos como,

$$
q_{L}(x)=\left[\begin{array}{c}
U_{L}(x) \\
D_{L}(x)
\end{array}\right], Q_{L}^{1}(x)=\left[\begin{array}{c}
U_{L}^{1}(x) \\
D_{L}^{1}(x)
\end{array}\right], Q_{R}^{1}(x)=\left[\begin{array}{c}
U_{R}^{1}(x) \\
D_{R}^{1}(x)
\end{array}\right] .
$$

Para os singletos de quarks temos $u_{R}(x)$ e $d_{R}(x)$ para os quarks do Modelo Padrão e $u_{R}^{1}(x), u_{L}^{1}(x)$ e $d_{R}^{1}(x), d_{L}^{1}(x)$ para os KK-quarks. Para os outro sabores de quarks charm e strange a obtenção do resultados é semelhante. Os estados que irão compor os KK-quarks e quarks são:

$$
q(x)=\left[\begin{array}{l}
q_{L}(x) \\
q_{R}(x)
\end{array}\right], Q_{L}^{1}(x)=\left[\begin{array}{c}
U_{L}^{1}(x) \\
D_{L}^{1}(x)
\end{array}\right], Q_{R}^{1}(x)=\left[\begin{array}{c}
U_{R}^{1}(x) \\
D_{R}^{1}(x)
\end{array}\right] .
$$

A lagrangiana gauge efetiva para nossa análise será:

$$
\begin{gathered}
\mathcal{L}_{\text {gauge }}=\frac{1}{2} \int_{-\pi R}^{+\pi R} d y\left[\frac{1}{4} B_{M N} B^{M N}-\frac{1}{4} G_{M N} G^{M N}\right], \\
\mathcal{L}_{\text {KK-quarks }}=\frac{1}{2} \int_{-\pi R}^{+\pi R} d y\left[i \bar{Q}(x, y) \Gamma^{M} D_{M} Q(x, y)+\right. \\
\left.+i \bar{U}(x, y) \Gamma^{M} D_{M} U(x, y)+i \bar{D}(x, y) \Gamma^{M} D_{M} D(x, y)\right] .
\end{gathered}
$$

Onde a derivada covariante é definida para os campos de matéria como:

$$
\begin{aligned}
& D_{M} Q(x, y)=\left(\partial_{M}+\frac{i y_{3} g_{1}^{(5)}}{2} B_{M}+i g_{3}^{(5)} G_{M}\right) Q(x, y), \\
& D_{M} U(x, y)=\left(\partial_{M}+\frac{i y_{4} g_{1}^{(5)}}{2} B_{M}+i g_{3}^{(5)} G_{M}\right) U(x, y), \\
& D_{M} D(x, y)=\left(\partial_{M}+\frac{i y_{5} g_{1}^{(5)}}{2} B_{M}+i g_{3}^{(5)} G_{M}\right) D(x, y) .
\end{aligned}
$$


A lagrangiana gauge A.50 contém os glúons e KK-glúons junto com fótons e KK-fótons. Os KK-fótons são estados diagonalizados de $B^{1}$ e $W_{3}^{1}$, o ângulo de Weinberg para essa mistura é pequeno quando $R^{-1}$ é grande, o que faz com que a primeira excitação KK para o fóton e bóson $Z$ seja basicamente os campos $B^{1}$ e $W_{3}^{1}$, respectivamente. Para exemplificar, o termo para os glúons e KK-glúons em A.50 pode ser expandido como,

$$
G_{M N} G^{M N}=G_{\mu \nu} G^{\mu \nu}+2 G_{5 \nu} G^{5 \nu} .
$$

Analisando somente os termos para o modo zero e primeiro estado excitado A.46, temos que o primeiro termo da equação acima fornece a lagrangiana gauge não-abeliana para o glúon $g_{\mu} \mathrm{e}$ KK-glúon $g_{\mu}^{(1)}$. O segundo fornece um termo de massa para $g^{(1)}$ do tipo,

$$
-\frac{1}{2}\left(\frac{1}{R^{2}}\right)\left(g_{\mu}^{(1)}\right)^{2}
$$

O mesmo resultado podemos obter para o fóton e KK-fóton. Ressaltamos que os modos zero dos campos não adquirem massa.

O termo de massa para os KK-quarks pode ser obtido de A.51, como exemplo realizamos a expansão para os dubletos $5 \mathrm{D}(Q)$,

$$
i \bar{Q}(x, y) \Gamma^{M} D_{M} Q(x, y)=i \bar{Q} \gamma^{\mu} D_{\mu} Q-\bar{Q} \gamma^{5} \partial_{y} Q .
$$

Onde usamos $G_{5}=0$. Após a integração na dimensão extra A.6. o primeiro termo da equação acima fornece parte da equação de movimento para os quarks Left sem massas e parte do termo cinético para os KK-quarks. O segundo termo fornece as massas dos KK-quarks,

$$
\bar{Q} \gamma^{5} \partial_{y} Q=-\frac{1}{R}\left(\bar{Q}_{L}^{1} Q_{R}^{1}+\bar{Q}_{R}^{1} Q_{L}^{1}\right)
$$

Assim como:

$$
\bar{U} \gamma^{5} \partial_{y} U=+\frac{1}{R}\left(\bar{U}_{L}^{1} U_{R}^{1}+\bar{U}_{R}^{1} U_{L}^{1}\right)
$$

O mesmo para $D(x, y)$. Para $U$ e $D$ temos um sinal oposto ao usual para massas de Dirac. Isso não será um problema pois a inserção do bóson de Higgs 5D junto com o acoplamento de Yukawa fornecem um mistura de estados que necessita de uma diagonalização para obter os estados físicos. Após essa definição teremos, por exemplo, os seguintes estados:

$$
\begin{gathered}
U^{(1)}=f\left(U_{L}^{1}, U_{R}^{1}\right), \\
u^{(1)}=f\left(u_{L}^{1}, u_{R}^{1}\right) .
\end{gathered}
$$

Com massa,

$$
m_{q_{t i p o-u}^{(1)}}=\sqrt{m_{u}^{2}+\frac{1}{R^{2}}}
$$

A mesmo resultado é válido para quarks do tipo down. As massas dos quarks em geral serão bem menores do que a contribuição $R^{-1}$, o que aparentemente leva a um quadro altamente degenerado para as massas dos KK-quarks a nível de árvore. Isso é resolvido nos cálculos de 1-loop para MUED, pois os campos 5D agora recebem contribuições quânticas diferentes, por exemplo um contra-termo $Z$ para as coordenadas espaço-tempo $(\mu)$ e um contra-termo $Z_{5}$ para a componente na quinta dimensão $(y)$. Enquanto a simetria de Lorentz em 5D é preservada, esses contra-termos são iguais e as correções são idênticas, levando a um quadro onde somente correções nas massas dos modos zero contribuam para a massa dos modos KK. Porém no orbifold temos a quebra da simetria de Lorentz em 5D, o que leva $Z$ e $Z_{5}$ a receberem contribuições diferentes. As 
contribuições de $Z_{5}$ influenciam diretamente na massa dos modos $\mathrm{KK}$, levando assim à quebra da degenerescência observada a nível de árvore 5 .

Usando as relações de matrizes $\Gamma$ da seção A.1, as expansões de campo A.46 e A.47), as derivadas covariantes, a identificação $g_{i}=\frac{g_{i}^{(5)}}{\sqrt{\pi R}}$ e denotando os dubletos de KK-quarks por $Q^{(1)}$ e os singletos de KK-quarks por $q^{(1)}$. Podemos determinar as lagrangianas relevantes para produção de jatos e MET.

$$
\begin{gathered}
\mathcal{L}_{\mathrm{KK}}=\mathcal{L}_{g q^{(1)} q^{(1)}}+\mathcal{L}_{g g^{(1)} g^{(1)}}+\mathcal{L}_{q q^{(1)} g^{(1)}}+\mathcal{L}_{q q^{(1)} B^{(1)}} . \\
\mathcal{L}_{g q^{(1)} q^{(1)}}=-g_{s} \bar{Q}_{R, L}^{(1)} \gamma^{\mu} g_{\mu} Q_{R, L}^{(1)}-g_{s} \bar{q}_{R, L}^{(1)} \gamma^{\mu} g_{\mu} q_{R, L}^{(1)}, \\
\mathcal{L}_{q q^{(1)} g^{(1)}}=-g_{s}\left[\bar{q}_{L} \gamma^{\mu} g_{\mu}^{(1)} Q_{L}^{(1)}+\bar{q}_{R} \gamma^{\mu} g_{\mu}^{(1)} q_{R}^{(1)}\right], \\
\left.+\left(\partial_{\mu} g_{\nu}^{(1), a}-\partial_{\nu} g_{\mu}^{(1), a}\right) g^{b \mu} g^{(1), c \nu}-\left(\partial_{\mu} g_{\nu}^{(1), b}-\partial_{\nu} g_{\mu}^{(1), b}\right) g^{a \mu} g^{c \nu}\right] . \\
\mathcal{L}_{g g^{(1)} g^{(1)}}=-\frac{g}{2} f^{a b c}\left[\left(\partial_{\mu} g_{\nu}^{a}-\partial_{\nu} g_{\mu}^{a}\right) g^{(1), b \mu} g^{(1), c \nu}\right. \\
\mathcal{L}_{q q^{(1)} B^{(1)}}=-g\left[y \bar{q}_{L} \gamma^{\mu} B_{\mu}^{(1)} Q_{L}^{(1)}+y^{\prime} \bar{q}_{R} \gamma^{\mu} B_{\mu}^{(1)} q_{R}^{(1)}\right] .
\end{gathered}
$$

No termo $\mathcal{L}_{q q^{(1)} B^{(1)}}$, y e $y^{\prime}$ são as hipercargas dos quarks $q_{L, R}$.

\section{A.4 Detalhes de Simulação}

Uma ressalva sobre a versão que utilizamos do MADGraPh5(2.2.3), ela está com um sério problema para as imagens de DJR e a análise de matching. Os kernels novos do linux 3.xx estão sendo disponibilizados com compiladores $\mathrm{C}++11$ e eles não são compatíveis totalmente com os compiladores C++ padrão usados nas rotinas do MADGRAPH5, portanto algumas mudanças devem ser feitas para distribuições linux com kernels novos. Além disso a versão atual do Rootestá apresentando um comportamento estranho no momento da análise das distribuições DJR, portanto recomenda-se retirar a passagem do ROOT no scritp create_matching_plots.sh e ajustar devidamente a rotina .C de mesmo nome para assim termos as figuras para a distribuição DJR. Espera-se que nas próximas versões esse problema seja resolvido.A produção inclusiva de eventos, realizada em nossa análise, exige a geração de jatos vindos de radiação de QCD. O programa Pyтнiaserá o responsável pelo parton shower (PS) e hadronização dos eventos. Porém, em nosso caso, poderemos produzir jatos muito energéticos e bem separados no detector, um regime onde geradores de eventos como o PүтніA precisam ser adaptados para um correto tratamento da transição entre os regimes soft e hard de emsissão de partons extras [85]. 


\section{Referências Bibliográficas}

[1] J. Wess, J. Bagger, "Supersymmetry and supergravity," Princeton University Press (1992).

[2] S. P. Martin, "A Supersymmetry primer," Adv. Ser. Direct. High Energy Phys. 21, 1 (2010) [Adv. Ser. Direct. High Energy Phys. 18, 1 (1998)] [hep-ph/9709356].

[3] H. E. Haber and G. L. Kane, "The Search for Supersymmetry: Probing Physics Beyond the Standard Model," Phys. Rept. 117, 75 (1985).

[4] A. Perez-Lorenzana, "An Introduction to extra dimensions," J. Phys. Conf. Ser. 18, 224 (2005) [hep-ph/0503177].

[5] H. C. Cheng, K. T. Matchev and M. Schmaltz, "Radiative corrections to Kaluza-Klein masses," Phys. Rev. D 66, 036005 (2002) [hep-ph/0204342].

[6] H. C. Cheng, K. T. Matchev and M. Schmaltz, "Bosonic supersymmetry? Getting fooled at the CERN LHC," Phys. Rev. D 66, 056006 (2002) [hep-ph/0205314].

[7] A. Datta, K. Kong and K. T. Matchev, "Minimal Universal Extra Dimensions in CalcHEP/CompHEP," New J. Phys. 12, 075017 (2010) [arXiv:1002.4624 [hep-ph]].

[8] C. G. Lester and D. J. Summers, "Measuring masses of semiinvisibly decaying particles pair produced at hadron colliders," Phys. Lett. B 463, 99 (1999) [hep-ph/9906349].

[9] P. Konar, K. Kong, K. T. Matchev and M. Park, "Superpartner Mass Measurement Technique using 1D Orthogonal Decompositions of the Cambridge Transverse Mass Variable $M_{T 2}$," Phys. Rev. Lett. 105, 051802 (2010) [arXiv:0910.3679 [hep-ph]].

[10] C. G. Lester, "Constrained invariant mass distributions in cascade decays: The Shape of the 'm(qll)-threshold' and similar distributions," Phys. Lett. B 655, 39 (2007) [hep-ph/0603171].

[11] J. M. Smillie and B. R. Webber, "Distinguishing spins in supersymmetric and universal extra dimension models at the large hadron collider," JHEP 0510, 069 (2005) [hep-ph/0507170].

[12] A. Alves and O. Eboli, "Unravelling the sbottom spin at the CERN LHC," Phys. Rev. D 75, 115013 (2007) [arXiv:0704.0254 [hep-ph]].

[13] A. J. Barr, "Measuring slepton spin at the LHC," JHEP 0602, 042 (2006) [hep-ph/0511115].

[14] T. Melia, "Spin before mass at the LHC," JHEP 1201, 143 (2012) [arXiv:1110.6185 [hep-ph]].

[15] A. T. Doyle, S. Ferrag, C. Wright, "Sensitivity Studies using ttH at the LHC," ATLAS notes (2008).

[16] R. Cousins, J. Mumford and V. Valuev, "Forward-backward asymmetry of simulated and reconstructed Z-prime $\longrightarrow$ mu+ mu- events in CMS," CERN-CMS-NOTE-2005-022.

[17] S. Brandt, "Data analysis: statistical and computational methods for scientists and engineers," Springer Science \& Business Media (2012). 
[18] F. Kling, T. Plehn and M. Takeuchi, "Tagging single Tops," Phys. Rev. D 86, 094029 (2012) [arXiv:1207.4787 [hep-ph]].

[19] J. M. Butterworth, A. R. Davison, M. Rubin and G. P. Salam, "Jet substructure as a new Higgs search channel at the LHC," Phys. Rev. Lett. 100, 242001 (2008) [arXiv:0802.2470 [hep-ph]].

[20] G. Aad et al. [ATLAS Collaboration], "Search for high-mass diboson resonances with bosontagged jets in proton-proton collisions at $\sqrt{s}=8 \mathrm{TeV}$ with the ATLAS detector," arXiv:1506.00962 [hep-ex].

[21] J. Gallicchio and M. D. Schwartz, "Quark and Gluon Tagging at the LHC," Phys. Rev. Lett. 107, 172001 (2011) [arXiv:1106.3076 [hep-ph]].

[22] G. Aad et al. [ATLAS Collaboration], "Light-quark and gluon jet discrimination in $p p$ collisions at $\sqrt{s}=7 \mathrm{TeV}$ with the ATLAS detector," Eur. Phys. J. C 74, no. 8, 3023 (2014) [arXiv:1405.6583 [hep-ex]].

[23] N. Fornengo, "Status and perspectives of indirect and direct dark matter searches," Adv. Space Res. 41, 2010 (2008) [astro-ph/0612786].

[24] E. Komatsu et al. [WMAP Collaboration], "Seven-Year Wilkinson Microwave Anisotropy Probe (WMAP) Observations: Cosmological Interpretation," Astrophys. J. Suppl. 192, 18 (2011) [arXiv:1001.4538 [astro-ph.CO]].

[25] K. Garrett and G. Duda, "Dark Matter: A Primer," Adv. Astron. 2011, 968283 (2011) [arXiv:1006.2483 [hep-ph]].

[26] S. F. King, "Neutrino mass," Contemp. Phys. 48, 195 (2007) [arXiv:0712.1750 [physics.pop$\mathrm{ph}]]$.

[27] Q. R. Ahmad et al. [SNO Collaboration], "Direct evidence for neutrino flavor transformation from neutral current interactions in the Sudbury Neutrino Observatory," Phys. Rev. Lett. 89, 011301 (2002) [nucl-ex/0204008].

[28] N. Jarosik et al., "Seven-Year Wilkinson Microwave Anisotropy Probe (WMAP) Observations: Sky Maps, Systematic Errors, and Basic Results," Astrophys. J. Suppl. 192, 14 (2011) [arXiv:1001.4744 [astro-ph.CO]].

[29] J. W. F. Valle, "Neutrino mass in supersymmetry," AIP Conf. Proc. 1200, 112 (2010) [arXiv:0911.3103 [hep-ph]].

[30] M. Tegmark et al. [SDSS Collaboration], "Cosmological parameters from SDSS and WMAP," Phys. Rev. D 69, 103501 (2004) [astro-ph/0310723].

[31] P. Huet and E. Sather, "Electroweak baryogenesis and standard model CP violation," Phys. Rev. D 51, 379 (1995) [hep-ph/9404302].

[32] J. M. Cline, M. Joyce and K. Kainulainen, "Supersymmetric electroweak baryogenesis," JHEP 0007, 018 (2000) [hep-ph/0006119].

[33] M. Peskin, D. Schroeder, "An introduction to quantum field theory," Westview (1995).

[34] G. Aad et al. [ATLAS Collaboration], "Observation of a new particle in the search for the Standard Model Higgs boson with the ATLAS detector at the LHC," Phys. Lett. B 716, 1 (2012) [arXiv:1207.7214 [hep-ex]]. 
[35] M. Schmaltz, "Physics beyond the standard model (theory): Introducing the little Higgs," Nucl. Phys. Proc. Suppl. 117, 40 (2003) [hep-ph/0210415].

[36] P. Nath and P. Fileviez Perez, "Proton stability in grand unified theories, in strings and in branes," Phys. Rept. 441, 191 (2007) [hep-ph/0601023].

[37] X. Tata, "What is supersymmetry and how do we find it?," In *Campos do Jordao 1997, Particles and fields* 404-492 [hep-ph/9706307].

[38] G. F. Giudice and R. Rattazzi, "Theories with gauge mediated supersymmetry breaking," Phys. Rept. 322, 419 (1999) [hep-ph/9801271].

[39] C. F. Berger, J. S. Gainer, J. L. Hewett and T. G. Rizzo, "Supersymmetry Without Prejudice," JHEP 0902, 023 (2009) [arXiv:0812.0980 [hep-ph]].

[40] R. C. Cotta, J. S. Gainer, J. L. Hewett and T. G. Rizzo, "Dark Matter in the MSSM," New J. Phys. 11, 105026 (2009) [arXiv:0903.4409 [hep-ph]].

[41] R. C. Cotta, K. T. K. Howe, J. L. Hewett and T. G. Rizzo, "Phenomenological minimal supersymmetric standard model dark matter searches on ice," Phys. Rev. D 85, 035017 (2012) [arXiv:1105.1199 [hep-ph]].

[42] W. Porod, "SPheno, a program for calculating supersymmetric spectra, SUSY particle decays and SUSY particle production at e+ e- colliders," Comput. Phys. Commun. 153, 275 (2003)

[43] B. C. Allanach, "SOFTSUSY: a program for calculating supersymmetric spectra," Comput. Phys. Commun. 143, 305 (2002)

[44] M. Cahill-Rowley, J. L. Hewett, A. Ismail and T. G. Rizzo, "Lessons and prospects from the pMSSM after LHC Run I," Phys. Rev. D 91, no. 5, 055002 (2015) [arXiv:1407.4130 [hep-ph]].

[45] T. Aaltonen et al. [CDF Collaboration], "Inclusive Search for Squark and Gluino Production in $p \bar{p}$ Collisions at $\sqrt{s}=1.96-\mathrm{TeV}, "$ Phys. Rev. Lett. 102, 121801 (2009) [arXiv:0811.2512 [hep-ex]].

[46] V. M. Abazov et al. [D0 Collaboration], "Search for squarks and gluinos in events with jets and missing transverse energy using $2.1 \mathrm{fb}^{-1}$ of $p \bar{p}$ collision data at $\sqrt{s}=1.96-\mathrm{TeV}$," Phys. Lett. B 660, 449 (2008) [arXiv:0712.3805 [hep-ex]].

[47] J. Abdallah et al. [DELPHI Collaboration], "Searches for supersymmetric particles in e+ ecollisions up to 208-GeV and interpretation of the results within the MSSM," Eur. Phys. J. C 31, 421 (2003) [hep-ex/0311019].

[48] A. Heister et al. [ALEPH Collaboration], "Absolute lower limits on the masses of selectrons and sneutrinos in the MSSM," Phys. Lett. B 544, 73 (2002) [hep-ex/0207056].

[49] V. Khachatryan et al. [CMS Collaboration], "Searches for supersymmetry using the $\mathrm{M}_{T 2}$ variable in hadronic events produced in pp collisions at $8 \mathrm{TeV}$," JHEP 1505, 078 (2015) [arXiv:1502.04358 [hep-ex]].

[50] R. Aaij et al. [LHCb Collaboration], "Implications of LHCb measurements and future prospects," Eur. Phys. J. C 73, no. 4, 2373 (2013) [arXiv:1208.3355 [hep-ex]].

[51] P. Bechtle et al., "Constrained Supersymmetry after two years of LHC data: a global view with Fittino," JHEP 1206, 098 (2012) [arXiv:1204.4199 [hep-ph]]. 
[52] J. Jaeckel, V. V. Khoze, T. Plehn and P. Richardson, "Travels on the squark-gluino mass plane," Phys. Rev. D 85, 015015 (2012) [arXiv:1109.2072 [hep-ph]].

[53] K. Hwang and J. E. Kim, "Orbifold compactification and related phenomenology," hep$\mathrm{ph} / 0411286$.

[54] T. Appelquist, H. C. Cheng and B. A. Dobrescu, "Bounds on universal extra dimensions," Phys. Rev. D 64, 035002 (2001) [hep-ph/0012100].

[55] R. J. Barlow, "Statistics: a guide to the use of statistical methods in the physical sciences," John Wiley \& Sons (1989).

[56] "Statistical methods in experimental physics," F. James, World Scientific (2006).

[57] L. Lyons "Statistics for nuclear and particle physicists," cambridge university press (1989).

[58] , G. Cowan, "Statistical data analysis," Oxford University Press (1998).

[59] K. Kong and K. T. Matchev, "Phenomenology of universal extra dimensions," AIP Conf. Proc. 903, 451 (2007) [hep-ph/0610057].

[60] G. Servant, "Status Report on Universal Extra Dimensions After LHC8," Mod. Phys. Lett. A 30, no. 15, 1540011 (2015) [arXiv:1401.4176 [hep-ph]].

[61] V. M. Abazov et al. [D0 Collaboration], "Search for universal extra dimensions in $p \bar{p}$ collisions," Phys. Rev. Lett. 108, 131802 (2012) [arXiv:1112.4092 [hep-ex]].

[62] V. M. Abazov et al. [D0 Collaboration], "Measurement of direct photon pair production cross sections in $p \bar{p}$ collisions at $\sqrt{s}=1.96 \mathrm{TeV}$," Phys. Lett. B 690, 108 (2010) [arXiv:1002.4917 [hep-ex]].

[63] G. Aad et al. [ATLAS Collaboration], "Search for high-mass resonances decaying to dilepton final states in pp collisions at $\mathrm{s}^{* *}(1 / 2)=7-\mathrm{TeV}$ with the ATLAS detector," JHEP 1211, 138 (2012) [arXiv:1209.2535 [hep-ex]].

[64] T. Kakuda, K. Nishiwaki, K. y. Oda and R. Watanabe, "Universal extra dimensions after Higgs discovery," Phys. Rev. D 88, 035007 (2013) [arXiv:1305.1686 [hep-ph]].

[65] A. Datta, A. Patra and S. Raychaudhuri, "Higgs Boson Decay Constraints on a Model with a Universal Extra Dimension," Phys. Rev. D 89, no. 9, 093008 (2014) [arXiv:1311.0926 [hep-ph]].

[66] T. Aaltonen et al. [CDF Collaboration], "Observation of Single Top Quark Production and Measurement of |Vtb| with CDF," Phys. Rev. D 82, 112005 (2010) [arXiv:1004.1181 [hepex]].

[67] A. Alloul, N. D. Christensen, C. Degrande, C. Duhr and B. Fuks, "FeynRules 2.0 - A complete toolbox for tree-level phenomenology," Comput. Phys. Commun. 185, 2250 (2014) [arXiv:1310.1921 [hep-ph]].

[68] E. Conte, B. Fuks and G. Serret, "MadAnalysis 5, A User-Friendly Framework for Collider Phenomenology," Comput. Phys. Commun. 184, 222 (2013) [arXiv:1206.1599 [hep-ph]].

[69] K. A. Olive et al. [Particle Data Group Collaboration], "Review of Particle Physics," Chin. Phys. C 38, 090001 (2014).

[70] G. Aad et al. [ATLAS Collaboration], "Study of the spin and parity of the Higgs boson in diboson decays with the ATLAS detector," arXiv:1506.05669 [hep-ex]. 
[71] W. Verkerke, "Pratical Statistics," NIKHEF - ATLAS.

[72] A. Albaid and K. S. Babu, "Higgs boson of mass $125 \mathrm{GeV}$ in GMSB models with messengermatter mixing," Phys. Rev. D 88, 055007 (2013) [arXiv:1207.1014 [hep-ph]].

[73] J. Hewett, "Supersymmetry Basics - The 2012 SLAC Summer Institute" (2012).

[74] H. Baer, V. Barger, D. Mickelson and M. Padeffke-Kirkland, "SUSY models under siege: LHC constraints and electroweak fine-tuning," Phys. Rev. D 89, no. 11, 115019 (2014) [arXiv:1404.2277 [hep-ph]].

[75] L. Edelhäuser, M. Krämer and J. Sonneveld, "Simplified models for same-spin new physics scenarios," JHEP 1504, 146 (2015) [arXiv:1501.03942 [hep-ph]].

[76] CMS Collaboration [CMS Collaboration], "Search for supersymmetry in hadronic final states using MT2 with the CMS detector at sqrt(s) $=8 \mathrm{TeV}$," CMS-PAS-SUS-13-019.

[77] K. i. Hikasa and Y. Nakamura, "Soft breaking correction to hard supersymmetric relations: QCD corrections to squark decay," Z. Phys. C 70, 139 (1996) [Z. Phys. C 71, 356 (1996)] [hep-ph/9501382].

[78] W. Beenakker, R. Hopker and P. M. Zerwas, "SUSY QCD decays of squarks and gluinos," Phys. Lett. B 378, 159 (1996) [hep-ph/9602378].

[79] P. Z. Skands et al., "SUSY Les Houches accord: Interfacing SUSY spectrum calculators, decay packages, and event generators," JHEP 0407, 036 (2004) [hep-ph/0311123].

[80] B. C. Allanach et al., "SUSY Les Houches Accord 2," Comput. Phys. Commun. 180, 8 (2009) [arXiv:0801.0045 [hep-ph]].

[81] J. Alwall, M. Herquet, F. Maltoni, O. Mattelaer and T. Stelzer, "MadGraph 5 : Going Beyond," JHEP 1106, 128 (2011) [arXiv:1106.0522 [hep-ph]].

[82] T. Sjostrand, S. Mrenna and P. Z. Skands, "PYTHIA 6.4 Physics and Manual," JHEP 0605, 026 (2006) [hep-ph/0603175].

[83] J. Conway et al., "PGS4: Pretty Good (Detector) Simulation" (2009).

[84] W. Beenakker, R. Hopker, M. Spira and P. M. Zerwas, "Squark and gluino production at hadron colliders," Nucl. Phys. B 492, 51 (1997) [hep-ph/9610490].

[85] P. Lenzi and J. M. Butterworth, "A Study on Matrix Element corrections in inclusive

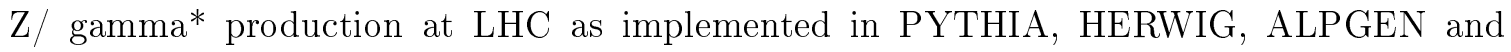
SHERPA," arXiv:0903.3918 [hep-ph].

[86] J. Alwall et al., "Comparative study of various algorithms for the merging of parton showers and matrix elements in hadronic collisions," Eur. Phys. J. C 53, 473 (2008) [arXiv:0706.2569 [hep-ph]].

[87] L. Randall and D. Tucker-Smith, "Dijet Searches for Supersymmetry at the LHC," Phys. Rev. Lett. 101, 221803 (2008) [arXiv:0806.1049 [hep-ph]].

[88] A. Barr, C. Lester and P. Stephens, "m(T2): The Truth behind the glamour," J. Phys. G 29, 2343 (2003) [hep-ph/0304226].

[89] O. J. P. Eboli, C. S. Fong, J. Gonzalez-Fraile and M. C. Gonzalez-Garcia, "Determination of the Spin of New Resonances in Electroweak Gauge Boson Pair Production at the LHC," Phys. Rev. D 83, 095014 (2011) [arXiv:1102.3429 [hep-ph]]. 
[90] L. Edelhäuser, J. Heisig, M. Krämer, L. Oymanns and J. Sonneveld, "Constraining supersymmetry at the LHC with simplified models for squark production," JHEP 1412, 022 (2014) [arXiv:1410.0965 [hep-ph]].

[91] G. Aad et al. [ATLAS Collaboration], "Search for squarks and gluinos with the ATLAS detector in final states with jets and missing transverse momentum using $\sqrt{s}=8 \mathrm{TeV}$ proton-proton collision data," JHEP 1409, 176 (2014) [arXiv:1405.7875 [hep-ex]].

[92] S. Chatrchyan et al. [CMS Collaboration], "Search for new physics in the multijet and missing transverse momentum final state in proton-proton collisions at $\sqrt{s}=8 \mathrm{TeV}$," JHEP 1406, 055 (2014) [arXiv:1402.4770 [hep-ex]].

[93] S. Chatrchyan et al. [CMS Collaboration], "Search for Supersymmetry at the LHC in Events with Jets and Missing Transverse Energy," Phys. Rev. Lett. 107, 221804 (2011) [arXiv:1109.2352 [hep-ex]].

[94] W. Beenakker, R. Hopker and M. Spira, "PROSPINO: A Program for the production of supersymmetric particles in next-to-leading order QCD," hep-ph/9611232.

[95] W. Hollik, J. M. Lindert, E. Mirabella and D. Pagani, "Electroweak corrections to squarkantisquark production at the LHC," JHEP 1508, 099 (2015) [arXiv:1506.01052 [hep-ph]].

[96] C. F. Berger, Z. Bern, L. J. Dixon, F. Febres Cordero, D. Forde, H. Ita, D. A. Kosower and D. Maitre, "An Automated Implementation of On-Shell Methods for One-Loop Amplitudes," Phys. Rev. D 78, 036003 (2008) [arXiv:0803.4180 [hep-ph]].

[97] C. Oleari and D. Zeppenfeld, "QCD corrections to electroweak nu(l) j j and l+ l- j j production," Phys. Rev. D 69, 093004 (2004) [hep-ph/0310156].

[98] L. T. Brady, A. Accardi, W. Melnitchouk and J. F. Owens, "Impact of PDF uncertainties at large x on heavy boson production," JHEP 1206, 019 (2012) [arXiv:1110.5398 [hep-ph]].

[99] G. Aad et al. [ATLAS Collaboration], "Improved luminosity determination in pp collisions at sqrt(s) $=7 \mathrm{TeV}$ using the ATLAS detector at the LHC," Eur. Phys. J. C 73, no. 8, 2518 (2013) [arXiv:1302.4393 [hep-ex]].

[100] P. Pralavorio, "Supersymmetry Searches - The 2012 SLAC Summer Institute" (2012).

[101] C. Carli, "LHC performance. Proceedings, Workshop, Chamonix, France, January 25-29, 2010."

[102] I. Hinchliffe, A. Kotwal, M. L. Mangano, C. Quigg and L. T. Wang, "Luminosity goals for a 100-TeV pp collider," Int. J. Mod. Phys. A 30, 1544002 (2015) [arXiv:1504.06108 [hep-ph]]. 